Supporting information to accompany:

\title{
Branched-chain and dendritic lipids for lipid nanoparticles
}

Michael W. Meanwell, Connor O'Sullivan, Perry Howard and Thomas M. Fyles

\section{Contents}

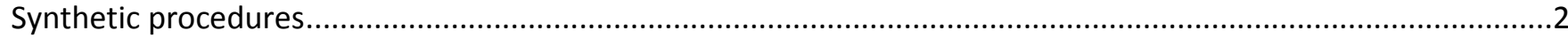

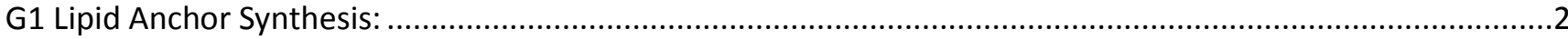

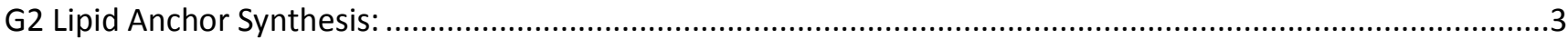

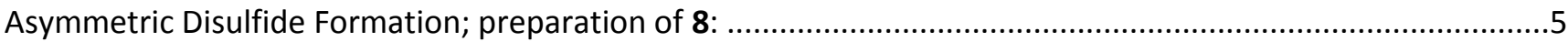

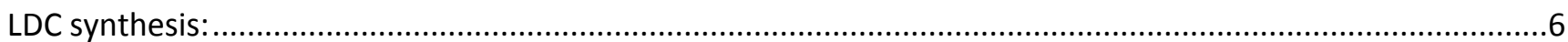

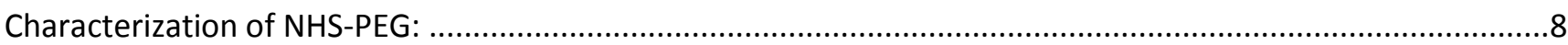

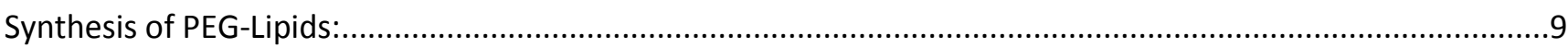

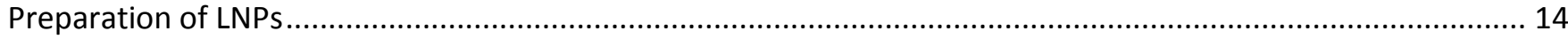

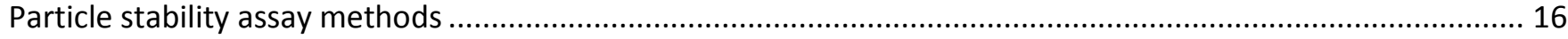

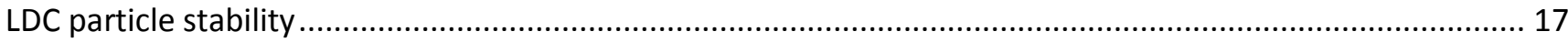

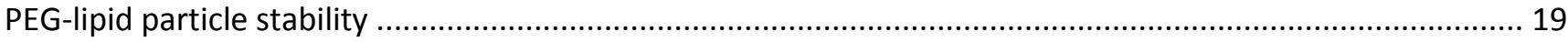

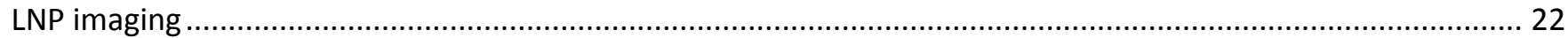

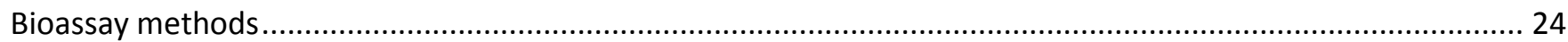

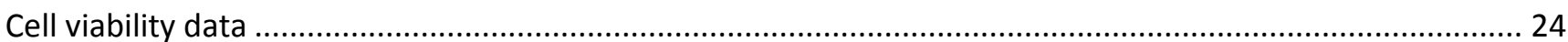

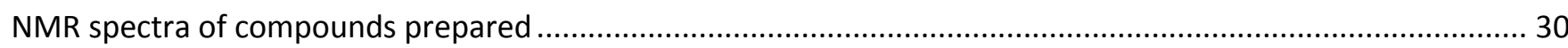




\section{Synthetic procedures}

Chemicals and solvents were used as received from known suppliers, except dry DCM which was obtained from a MBRAUN-SPS. NMR spectra were collected on a $300 \mathrm{MHz}$ Bruker instrument. ESI-MS spectra were recorded on a LCQ-Classic instrument.

\section{G1 Lipid Anchor Synthesis:}
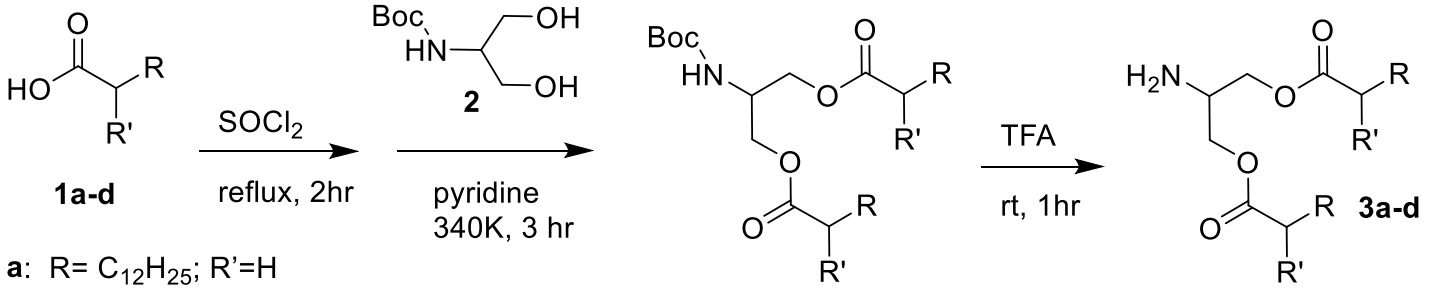

b: $\mathrm{R}=\mathrm{C}_{14} \mathrm{H}_{29} ; \mathrm{R}^{\prime}=\mathrm{H}$

c: $\mathrm{R}=\mathrm{C}_{8} \mathrm{H}_{17} ; \mathrm{R}^{\prime}=\mathrm{C}_{6} \mathrm{H}_{13}$

d: $\mathrm{R}=\mathrm{C}_{16} \mathrm{H}_{33} ; \mathrm{R}^{\prime}=\mathrm{H}$

\section{Scheme S1: Outline of the synthesis for the G1 lipid anchor}

General Procedure: In a round bottom flask a stirred solution of thionyl chloride (10.0 equiv.) and 1a-d (1.0 equiv.) was refluxed for 2 hours under a $\mathrm{CaSO}_{4}$ drying tube. The reaction was monitored by ${ }^{1} \mathrm{H}-\mathrm{NMR}$ analysis of aliquots. The reaction was allowed to cool and subsequently the thionyl chloride was removed under vacuum on a rotoevaporator to obtain the acid chloride. In a round bottom flask 2 (1.0 equiv.) was dissolved in pyridine (10.0 equiv.) and the acid chloride ( 2.5 equiv.) was added dropwise. The mixture was then heated to $70^{\circ} \mathrm{C}$ and left to stir for 3 hours under a $\mathrm{CaSO}_{4}$ drying tube. The reaction was monitored by ${ }^{1} \mathrm{H}-\mathrm{NMR}$ analysis of aliquots. Once completed, the reaction was cooled and diluted with DCM. The solution was then washed 3 times with $1 \mathrm{M} \mathrm{HCl}$ and 3 times with $1 \mathrm{M} \mathrm{NaOH}$. The organic layer was then dried over anhydrous sodium sulfate and then gravity filtered. The solvent was removed under vacuum to obtain the Boc-protected anchor. Excess TFA was added dropwise to the Boc-protected anchor in a round bottom flask. The reaction was left stirring for 1 hour at room temperature and was monitored by TLC (silica gel, EtOAc/ Hexanes as eluent, visualized by $\mathrm{KMnO}_{4}$ ). Following completion of the reaction, TFA was removed on a roto-evaporator. $\mathrm{NaOH}(1 \mathrm{M})$ was then added and 3a-d was extracted with DCM and washed 3 times with $1 \mathrm{M} \mathrm{NaOH}$. The organic layer was dried with anhydrous sodium carbonate and subsequently gravity filtered. DCM was removed on a roto-evaporator yielding $\mathbf{3 a - d}$. No chromatography was necessary.

3a: By the general procedure: step 1, thionyl chloride $(6.35 \mathrm{~mL}, 87.6 \mathrm{mmol})$ and $1 \mathrm{a}(2.000 \mathrm{~g}, 8.757 \mathrm{mmol})$; step 2, $2(0.596 \mathrm{~g}, 3.13 \mathrm{mmol})$ in pyridine $(2.53 \mathrm{~mL}, 31.3 \mathrm{mmol})$ and the acid chloride $(1.931 \mathrm{~g}, 7.835 \mathrm{mmol})$; step 3, TFA $(5 \mathrm{~mL})$ and the product of step 2; as a waxy yellow solid in a $66 \%$ yield $(1.06 \mathrm{~g}) .{ }^{1} \mathrm{H}-\mathrm{NMR}\left(300 \mathrm{MHz}, \mathrm{CDCl}_{3}\right) \delta: 4.07$ $(\mathrm{m}, 2 \mathrm{H}), 4.05(\mathrm{~m}, 2 \mathrm{H}), 3.28(\mathrm{~m}, 1 \mathrm{H}), 2.33(\mathrm{t}, 4 \mathrm{H}, 7.7 \mathrm{~Hz}), 1.62(\mathrm{~m}, 4 \mathrm{H}), 1.48(\mathrm{~s}(\mathrm{br}), 2 \mathrm{H}), 1.26(\mathrm{~s}, 40 \mathrm{H}), 0.88(\mathrm{t}, 6 \mathrm{H}, 7.0$ Hz). ${ }^{13} \mathrm{C}-\mathrm{NMR}\left(75 \mathrm{MHz}_{2} \mathrm{CDCl}_{3}\right):$ 173.6, 65.8, 49.3, 34.2, 31.9, 29.6, 29.6, 20.4, 29.3, 29.2, 29.1, 24.9, 22.7, $14.1 \mathrm{MS}$ ((+) ESI): calc'd for $\mathrm{C}_{31} \mathrm{H}_{62} \mathrm{NO}_{4}{ }^{+}=512.47 \mathrm{amu}$, obtained $=512.33 \mathrm{amu}$.

3b: By the general procedure: step 1, thionyl chloride $(5.61 \mathrm{~mL}, 78.0 \mathrm{mmol})$ and $\mathbf{1 b}(2.000 \mathrm{~g}, 7.800 \mathrm{mmol})$; step 2, $2(0.558 \mathrm{~g}, 2.92 \mathrm{mmol})$ in pyridine $(2.31 \mathrm{~mL}, 29.2 \mathrm{mmol})$ and the acid chloride $(2.000 \mathrm{~g}, 7.299 \mathrm{mmol})$; step 3 TFA $(5 \mathrm{~mL})$ and the product of step 2; as a waxy white solid in a 73\% yield (1.21 g). ${ }^{1} \mathrm{H}-\mathrm{NMR}\left(300 \mathrm{MHz}, \mathrm{CDCl}_{3}\right) \delta: 4.06$ $(\mathrm{m}, 2 \mathrm{H}), 4.05(\mathrm{~m}, 2 \mathrm{H}), 3.28(\mathrm{~m}, 1 \mathrm{H}), 2.32(\mathrm{t}, 4 \mathrm{H}, \mathrm{J}=7.8 \mathrm{~Hz}), 1.61(\mathrm{~m}, 4 \mathrm{H}), 1.24(\mathrm{~s}, 48 \mathrm{H}), 0.87(\mathrm{t}, 6 \mathrm{H}) .{ }^{13} \mathrm{C}-\mathrm{NMR}(75$ 
$\left.\mathrm{MHz}, \mathrm{CDCl}_{3}\right) \delta: 173.8,66.0,49.5,34.4,32.1,29.9,29.9,29.8,29.7,29.6,29.5,29.4,25.2,22.9,14.3$. MS ((+) ESI): calc'd for $\mathrm{C}_{35} \mathrm{H}_{70} \mathrm{NO}_{4}^{+}=568.53 \mathrm{amu}$, obtained $=568.33 \mathrm{amu}$.

3c: By the general procedure: step 1, thionyl chloride $(5.66 \mathrm{~mL}, 78.2 \mathrm{mmol})$ and $1 \mathrm{c}(2.30 \mathrm{~mL}, 7.82 \mathrm{mmol})$; step 2, $2(0.488 \mathrm{~g}, 2.55 \mathrm{mmol})$ in pyridine $(2.46 \mathrm{~mL}, 30.6 \mathrm{mmol})$ and the acid chloride $(2.10 \mathrm{~g}, 7.64 \mathrm{mmol})$; step 3, TFA (5 $\mathrm{mL}$ ) and the product of step 2; as a light yellow oil in a 75\% yield (1.09 g). ${ }^{1} \mathrm{H}-\mathrm{NMR}\left(300 \mathrm{MHz}, \mathrm{CDCl}_{3}\right) \delta: 4.06(\mathrm{~m}$, $2 \mathrm{H}), 4.04(\mathrm{~m}, 2 \mathrm{H}), 3.26(\mathrm{~m}, 1 \mathrm{H}), 2.34(\mathrm{~m}, 2 \mathrm{H}), 1.57,1.44(\mathrm{~m}, 8 \mathrm{H}), 1.24(\mathrm{~s}, 40 \mathrm{H}), 0.86(\mathrm{t}, 12 \mathrm{H}, \mathrm{J}=7.0 \mathrm{~Hz}) .{ }^{13} \mathrm{C}-\mathrm{NMR}$ (75 MHz, $\left.\mathrm{CDCl}_{3}\right):$ 176.2, 65.5, 53.4, 49.4, 45.7, 32.4, 31.8, 31.6, 29.5, 29.4, 29.2, 29.2, 27.4, 27.4, 22.6, 22.6, 14.0, 14.0. $\mathrm{MS}((+) \mathrm{ESI})$ : calc'd for $\mathrm{C}_{35} \mathrm{H}_{70} \mathrm{NO}_{4}{ }^{+}=568.53 \mathrm{amu}$, obtained $=568.33 \mathrm{amu}$.

3d: By the general procedure: step 1, thionyl chloride $(5.10 \mathrm{~mL}, 70.3 \mathrm{mmol})$ and $\mathbf{1 d}(2.000 \mathrm{~g}, 7.030 \mathrm{mmol})$; step 2, $2(0.510 \mathrm{~g}, 2.67 \mathrm{mmol})$ in pyridine $(2.15 \mathrm{~mL}, 26.7 \mathrm{mmol})$ and the acid chloride $(2.020 \mathrm{~g}, 6.668 \mathrm{mmol})$; step 3, TFA $(5 \mathrm{~mL})$ and the product of step 2; as a waxy white solid in a $67 \%$ yield $(1.09 \mathrm{~g}) .{ }^{1} \mathrm{H}-\mathrm{NMR}\left(300 \mathrm{MHz}, \mathrm{CDCl}_{3}\right) \delta: 4.09$ $(\mathrm{m}, 2 \mathrm{H}), 4.07(\mathrm{~m}, 2 \mathrm{H}), 3.30(\mathrm{~m}, 1 \mathrm{H}), 2.34(\mathrm{t}, 4 \mathrm{H}, \mathrm{J}=7.5 \mathrm{~Hz}), 1.63(\mathrm{~m}, 4 \mathrm{H}), 1.26(\mathrm{~s}, 56 \mathrm{H}), 0.89(\mathrm{t}, 6 \mathrm{H}, \mathrm{J}=6.8 \mathrm{~Hz}) . \mathrm{MS}((+)$ ESI): calc'd for $\mathrm{C}_{39} \mathrm{H}_{78} \mathrm{NO}_{4}{ }^{+}=624.59 \mathrm{amu}$, obtained $=624.53 \mathrm{amu}$.

\section{G2 Lipid Anchor Synthesis:}

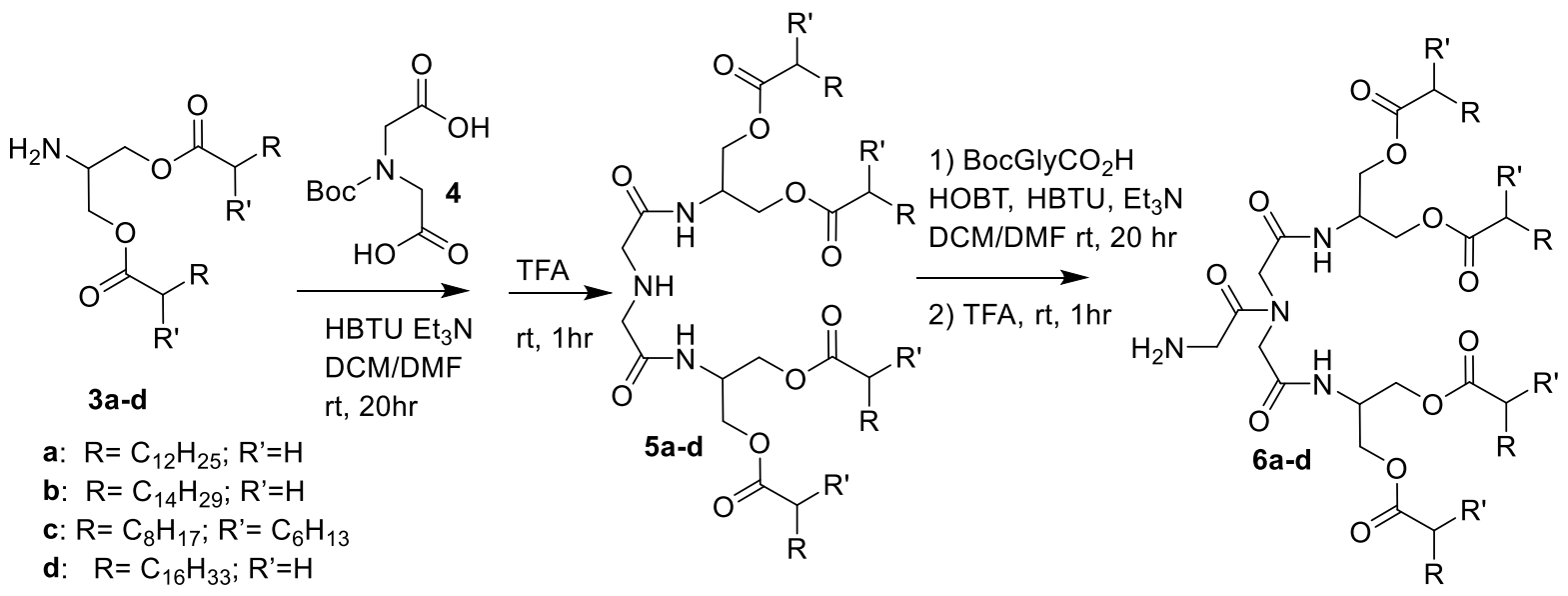

Scheme S2: Outline of the synthesis for the G2 lipid anchor

General Procedure: In a round bottom flask was dissolved 4 (1.0 equiv.) in dried DCM/DMF (1:1;10mM). HBTU (2.4 equiv.), 3a-d ( 2.2 equiv.) and triethylamine (4.0 equiv.) were subsequently added. The solution was stirred for 20 hours at room temperature and was monitored by ${ }^{1} \mathrm{H}-\mathrm{NMR}$. Once completed, the reaction solution was diluted with EtOAc and washed 3 times with $1 \mathrm{M} \mathrm{HCl}$ and 3 times with $\mathrm{H}_{2} \mathrm{O}$. The organic layer was then dried over anhydrous sodium sulfate, followed by gravity filtration. EtOAc and DCM were roto-evaporated to yield a Bocprotected product. Excess TFA was added dropwise to the Boc-protected product in a round bottom flask. The reaction was left to stir for 1 hour and monitored by ${ }^{1} \mathrm{H}-\mathrm{NMR}$. Following completion of the reaction, TFA was removed on roto-evaporator and $1 \mathrm{M} \mathrm{NaOH}$ was added to the round bottom flask, the mixture was extracted with DCM and washed 3 times with $1 \mathrm{M} \mathrm{NaOH}$. The organic layer was then dried over sodium carbonate, followed by gravity filtration. DCM was removed on a roto-evaporator and $\mathbf{5 a - d}$ was isolated. The crude product was directly characterized; no purification was necessary. [Characterization- ${ }^{1} \mathrm{H}-\mathrm{NMR},{ }^{13} \mathrm{C}-\mathrm{NMR}, \mathrm{ESI}-\mathrm{MS}$ ] In a round bottom flask was dissolved Boc-glycine (1.1 equiv.) in dried DCM/DMF (1:1; 10mM). To this, HOBt (1.2 equiv.), HBTU (1.2 equiv.), 5a-d (1.0 equiv.), and triethylamine (3.0 equiv.) were then added and the resulting solution was left stirring at room temperature for 20 hours. When the reaction was complete, visualized by ${ }^{1} \mathrm{H}$ - 
NMR, the reaction solution was diluted with EtOAc, and washed once with $1 \mathrm{M} \mathrm{HCl}$ and 3 times with $\mathrm{H}_{2} \mathrm{O}$. The organic layer was then dried over anhydrous sodium sulfate and then gravity filtered. DCM and EtOAc were removed by roto-evaporation to yield a crude Boc-protected. Excess TFA was added in a round bottom flask and the reaction was left for 1 hour. Following completion, as monitored by ${ }^{1} \mathrm{H}-\mathrm{NMR}$, TFA was removed on a rotoevaporator. $\mathrm{NaOH}(1 \mathrm{M})$ was added to convert to the amine and 6a-d was extracted with diethyl ether and subsequently washed with 3 times with $1 \mathrm{M} \mathrm{NaOH}$. The organic layer was dried with anhydrous sodium carbonate and then gravity filtered. Diethyl ether was removed to obtain the crude product.

5a and 6a: By the general procedure: step 1, 4(0.104 g, $0.444 \mathrm{mmol})$ in dried DCM/DMF $(22.2 \mathrm{~mL} / 22.2 \mathrm{~mL} ; 10$ $\mathrm{mM})$. HBTU (0.404 g, $1.066 \mathrm{mmol}), 3 \mathrm{a}(0.500 \mathrm{~g}, 0.977 \mathrm{mmol})$ and triethylamine $(0.25 \mathrm{~mL}, 1.8 \mathrm{mmol})$; step 2, TFA $(5 \mathrm{~mL})$ and the product of step1; a waxy light yellow solid in $53 \%$ yield $(0.264 \mathrm{~g})$. Data for $5 \mathrm{a}:{ }^{1} \mathrm{H}-\mathrm{NMR}(300 \mathrm{MHz}$, $\left.\mathrm{CDCl}_{3}\right)$ 8: $7.02(\mathrm{~d}, 2 \mathrm{H}, \mathrm{J}=8.6 \mathrm{~Hz}), 4.46(\mathrm{~m}, 2 \mathrm{H}), 4.24(\mathrm{~m}, 4 \mathrm{H}), 4.12(\mathrm{~m}, 4 \mathrm{H}), 3.25(\mathrm{~s}, 4 \mathrm{H}), 2.31(\mathrm{t}, 8 \mathrm{H}, \mathrm{J}=7.8 \mathrm{~Hz}), 1.59$ $(\mathrm{m}, 8 \mathrm{H}), 1.25(\mathrm{~s}, 80 \mathrm{H}), 0.87(\mathrm{t}, 12 \mathrm{H}, \mathrm{J}=6.9 \mathrm{~Hz}) .{ }^{13} \mathrm{C}-\mathrm{NMR}\left(75 \mathrm{MHz}, \mathrm{CDCl}_{3}\right)$ 8: 174.0, 170.8, 62.9, 52.8, 48.0, 34.3, 32.1, 29.9, 29.9, 29.7, 29.6, 29.5, 29.4, 25.1, 22.9, 14.3. MS ((+) ESI): calc'd for $\mathrm{C}_{66} \mathrm{H}_{126} \mathrm{~N}_{3} \mathrm{O}_{10}{ }^{+}=1120.94$ amu, obtained $=1121.00 \mathrm{amu}$. Continuing the general procedure: step 3, Boc-glycine $(0.033 \mathrm{~g}, 0.19 \mathrm{mmol})$ in dried DCM/DMF ( $9.0 \mathrm{~mL} / 9.0 \mathrm{~mL} ; 10 \mathrm{mM}), \mathrm{HOBt}(0.029 \mathrm{~g}, 0.21 \mathrm{mmol}), \mathrm{HBTU}(0.080 \mathrm{~g}, 0.21 \mathrm{mmol}), 5 \mathrm{a}$ (0.20 g, 0.179 $\mathrm{mmol})$, and triethylamine $(0.075 \mathrm{~mL}, 0.53 \mathrm{mmol})$; step 4, TFA $(5 \mathrm{~mL})$ and the Boc-protected product gave a light brown solid used without additional purification or characterization.

5b and 6b: By the general procedure: step1, $4(0.093 \mathrm{~g}, 0.40 \mathrm{mmol})$ in dried DCM/DMF $(20.0 \mathrm{~mL} / 20.0 \mathrm{~mL} ; 10$ $\mathrm{mM})$. HBTU (0.364 g, $0.960 \mathrm{mmol}), 3 \mathrm{~b}(0.500 \mathrm{~g}, 0.880 \mathrm{mmol})$ and triethylamine $(0.22 \mathrm{~mL}, 1.6 \mathrm{mmol})$; step 2, TFA $(5 \mathrm{~mL})$ and the Boc-protected product of step 1; as a light brown waxy solid in $55 \%$ yield $(0.272 \mathrm{~g})$. Data for $5 \mathbf{b}$ ${ }^{1} \mathrm{H}-\mathrm{NMR}\left(300 \mathrm{MHz}, \mathrm{CDCl}_{3}\right)$ 8: $7.03(\mathrm{~d}, 2 \mathrm{H}, \mathrm{J}=8.7 \mathrm{~Hz}), 4.47(\mathrm{~m}, 2 \mathrm{H}), 4.26(\mathrm{~m}, 4 \mathrm{H}), 4.14(\mathrm{~m}, 4 \mathrm{H}), 3.27(\mathrm{~s}, 4 \mathrm{H}), 2.33(\mathrm{t}$, $8 \mathrm{H}, \mathrm{J}=7.3 \mathrm{~Hz}), 1.60(\mathrm{~m}, 8 \mathrm{H}), 1.27(\mathrm{~s}, 96 \mathrm{H}), 0.89(\mathrm{t}, 12 \mathrm{H}, \mathrm{J}=6.8) .{ }^{13} \mathrm{C}-\mathrm{NMR}\left(75 \mathrm{MHz}, \mathrm{CDCl}_{3}\right) \delta: 174.0,170.8,62.8$, 52.7, 48.0, 34.31, 32.1, 29.9, 29.7, 29.6, 29.5, 29.4, 25.1, 22.9, 14.3. MS ((+) ESI): calc'd for $\mathrm{C}_{74} \mathrm{H}_{142} \mathrm{~N}_{3} \mathrm{O}_{10}{ }^{+}=$ $1233.07 \mathrm{amu}$, obtained $=1233.00$. Continuing the general procedure: step 3, Boc-glycine $(0.030 \mathrm{~g}, 0.17 \mathrm{mmol})$ in dried DCM/DMF (8.1 mL/8.1 mL; 10mM), HOBt (0.026 g, $0.20 \mathrm{mmol})$, HBTU (0.074 g, $0.20 \mathrm{mmol})$, 5b: (0.201, $0.162 \mathrm{mmol})$, and triethylamine $(0.068 \mathrm{~mL}, 0.49 \mathrm{mmol})$; step 4, TFA $(5 \mathrm{~mL})$ and the Boc-protected product of step 3 gave a light brown oil used without further purification. $\mathrm{MS}((+) \mathrm{ESI})$ : calc'd for $\mathrm{C}_{76} \mathrm{H}_{145} \mathrm{~N}_{4} \mathrm{O}_{11}{ }^{+}=1290.09$ amu, obtained $=1290.00 \mathrm{amu}$.

5c and 6c: By the general procedure: step 1, 4 (0.093 g, $0.40 \mathrm{mmol})$ in dried DMF (20.0 mL), HBTU (0.363 g, $0.960 \mathrm{mmol}), 3 \mathrm{c}(0.500 \mathrm{~g}, 0.880 \mathrm{mmol})$ and triethylamine $(0.22 \mathrm{~mL}, 1.6 \mathrm{mmol})$; step 2, TFA $(5 \mathrm{~mL})$ and the Bocprotected product of step 1; as a colorless oil in $60 \%$ yield $(0.296 \mathrm{~g})$. Data for $5 \mathrm{c}:{ }^{1} \mathrm{H}-\mathrm{NMR}\left(300 \mathrm{MHz}, \mathrm{CDCl}_{3}\right) \delta$ : $7.07(\mathrm{~d}, 2 \mathrm{H}, \mathrm{J}=8.6 \mathrm{~Hz}), 4.46(\mathrm{~m}, 2 \mathrm{H}), 4.25(\mathrm{~m}, 4 \mathrm{H}), 4.11(\mathrm{~m}, 4 \mathrm{H}), 3.23(\mathrm{~s}, 4 \mathrm{H}), 2.34(\mathrm{~m}, 4 \mathrm{H}), 1.56,1.45(\mathrm{~m}, 16 \mathrm{H})$, $1.24(\mathrm{~s}, 80 \mathrm{H}), 0.86$ (t, $24 \mathrm{H}, \mathrm{J}=7.4 \mathrm{~Hz}) .{ }^{13} \mathrm{C}-\mathrm{NMR}\left(75 \mathrm{MHz}, \mathrm{CDCl}_{3}\right)$ 8: 176.4, 170.4, 62.3, 52.6, 48.0, 45.6, 34.6, 34.4, 32.9, 32.7, 32.5, 32.3, 32.2, 31.8, 31.6, 31.5, 29.6, 29.5, 29.4, 29.2, 29.2, 29.0, 27.4, 27.4, 25.2, 22.6, 22.6, 20.6, 14.0, 14.0, 11.3. $\mathrm{MS}((+) \mathrm{ESI})$ : calc'd for $\mathrm{C}_{74} \mathrm{H}_{142} \mathrm{~N}_{3} \mathrm{O}_{10}{ }^{+}=1233.07 \mathrm{amu}$, obtained $=1232.87 \mathrm{amu}$. Continuing the general procedure: Boc-glycine $(0.030 \mathrm{~g}, 0.17 \mathrm{mmol})$ in dried DCM $(1.62 \mathrm{~mL}), \mathrm{HOBt}(0.026 \mathrm{~g}, 0.20 \mathrm{mmol})$, HBTU $(0.074 \mathrm{~g}, 0.20 \mathrm{mmol}), 5 \mathrm{c}(0.200 \mathrm{~g}, 0.162 \mathrm{mmol})$, and triethylamine $(0.068 \mathrm{~mL}, 0.49 \mathrm{mmol}) ;$ step 4, TFA $(5 \mathrm{~mL})$ and the Boc-protected product of step 3 gave a light brown oil used without further purification. MS ((+) ESI): calc'd for $\mathrm{C}_{76} \mathrm{H}_{145} \mathrm{~N}_{4} \mathrm{O}_{11}{ }^{+}=1290.09 \mathrm{amu}$, obtained $=1289.93 \mathrm{amu}$.

5d and 6d: By the general procedure: step 1, 4 (0.088 g, $0.36 \mathrm{mmol})$ in dried DCM/DMF (18.2 mL/18.2 mL; 10 $\mathrm{mM}), \mathrm{HBTU}(0.329 \mathrm{~g}, 0.867 \mathrm{mmol}), 5 \mathrm{~d}(0.500 \mathrm{~g}, 0.801 \mathrm{mmol})$ and triethylamine $(0.20 \mathrm{~mL}, 1.5 \mathrm{mmol})$; step 2, TFA $(5 \mathrm{~mL})$ and the Boc-protected product of step 1 ; as a white waxy solid in a $51 \%$ yield $(0.248 \mathrm{~g})$. Data for $5 \mathrm{~d}:{ }^{1} \mathrm{H}-$ 
NMR (300 MHz, CDCl $)$ ) $: 7.07(\mathrm{~d}, 2 \mathrm{H}, \mathrm{J}=8.6 \mathrm{~Hz}), 4.46(\mathrm{~m}, 2 \mathrm{H}), 4.25(\mathrm{~m}, 4 \mathrm{H}), 4.13(\mathrm{~m}, 4 \mathrm{H}), 3.26(\mathrm{~s}, 4 \mathrm{H}), 2.32(\mathrm{t}, 8 \mathrm{H}$, $\mathrm{J}=7.8 \mathrm{~Hz}), 1.60(\mathrm{~m}, 8 \mathrm{H}) 1.25(\mathrm{~s}, 112 \mathrm{H}), 0.87(\mathrm{t}, 12 \mathrm{H}, \mathrm{J}=6.8 \mathrm{~Hz}) .{ }^{13} \mathrm{C}-\mathrm{NMR}\left(75 \mathrm{MHz}, \mathrm{CDCl}_{3}\right) \delta:$ 174.0, 170.8, 62.9, $52.8,48.1,34.3,32.1,29.9,29.7,29.6,29.5,29.4,25.1,22.9,14.3$. MS ((+) ESI): calc'd for $\mathrm{C}_{82} \mathrm{H}_{158} \mathrm{~N}_{3} \mathrm{O}_{10}{ }^{+}=1345.19$ amu, obtained $=1345.07 \mathrm{amu}$. Continuing the general procedure: step 3, Boc glycine $(0.013 \mathrm{~g}, 0.074 \mathrm{mmol})$ in dried DCM/DMF (3.7 mL/ $3.7 \mathrm{~mL} ; 10 \mathrm{mM}), \mathrm{HOBt}(0.012 \mathrm{~g}, 0.085 \mathrm{mmol}), \mathrm{HBTU}(0.032 \mathrm{~g}, 0.085 \mathrm{mmol}), 5 \mathrm{~d}(0.0950$ $\mathrm{g}, 0.0706 \mathrm{mmol})$, and triethylamine $(0.030 \mathrm{~mL}, 0.21 \mathrm{mmol})$; step 4, TFA $(5 \mathrm{~mL})$ and the Boc-protected product of step 3 gave a light brown oil used without further purification.

\section{Asymmetric Disulfide Formation; preparation of 8:}

In a round bottom flask was dissolved 6-mercaptopurine monohydrate $(0.500 \mathrm{~g}, 2.94 \mathrm{mmol})$ and $11-$

mercaptoundecanoic acid $(0.641 \mathrm{~g}, 2.94 \mathrm{mmol})$ in DMSO $(9.0$ $\mathrm{mL} ; 0.33 \mathrm{M})$. While the reaction was stirred, solid DDQ $(0.669$ $\mathrm{g}, 2.94 \mathrm{mmol}$ ) was slowly added over 10 minutes and then left at room temperature for 1 hour. The reaction was monitored by TLC (silica gel, MeOH/DCM as eluent, visualized by iodine).

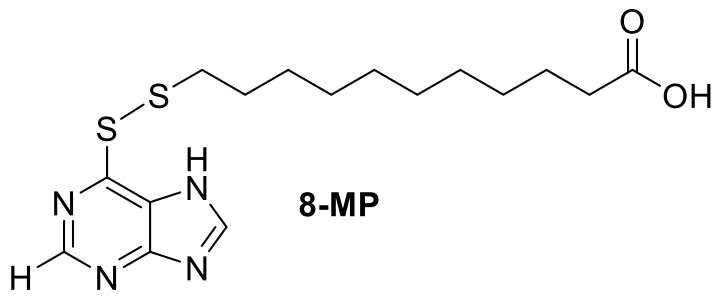

Following completion, the product was precipitated by adding $20 \mathrm{~mL}$ water to the reaction solution. The resulting mixture was left for 6 hours before being vacuum filtered and a red solid was isolated. The crude product was redissolved in hot methanol and cooled in an ice water bath then vacuum filtered. A white powder was afforded in a 60\% yield (0.622 g). ${ }^{1} \mathrm{H}-\mathrm{NMR}\left(300 \mathrm{MHz}, \mathrm{DMSO}-\mathrm{d}^{6}\right) \delta: 8.84(\mathrm{~s}, 1 \mathrm{H}), 8.57(\mathrm{~s}, 1 \mathrm{H}), 2.96(\mathrm{t}, 2 \mathrm{H}, \mathrm{J}=7.0$ $\mathrm{Hz}$ ), $2.21(\mathrm{t}, 2 \mathrm{H}, \mathrm{J}=7.3 \mathrm{~Hz}), 1.67$ (quintet, $2 \mathrm{H}, \mathrm{J}=7.5 \mathrm{~Hz}), 1.50,1.40(\mathrm{~m}, 4 \mathrm{H}), 1.25(\mathrm{~s}, 10 \mathrm{H}) .{ }^{13} \mathrm{C}-\mathrm{NMR}(75 \mathrm{MHz}$, DMSO-d $\left.{ }^{6}\right)$ 8: 174.4, 151.7, (broad low intensity peaks), 39.5, 38.1, 33.6, 28.8, 28.7, 28.5, 28.1, 27.6, 24.5. (MS ((+) ESI): calc'd for $\mathrm{C}_{16} \mathrm{H}_{25} \mathrm{~N}_{4} \mathrm{O}_{2} \mathrm{~S}_{2}{ }^{+}=369.14 \mathrm{amu}$, obtained $=369.13 \mathrm{amu}$.

Similarly, in a round bottom flask was dissolved 6thioguanine $(0.500 \mathrm{~g}, 2.99 \mathrm{mmol})$ and $11-$ mercaptoundecanoic acid (0.652 $\mathrm{g}, 2.99 \mathrm{mmol})$ in DMSO $(0.15 \mathrm{M})$. While the reaction was stirred, solid DDQ (0.679 g, $2.99 \mathrm{mmol}$ ) was slowly added over 10 minutes and then left at room temperature for 1 hour. The reaction was monitored by TLC (silica gel, $\mathrm{MeOH} / \mathrm{DCM}$<smiles>Nc1nc(SSCCCCCCCCCCC(=O)O)c2[nH]cnc2n1</smiles>
as eluent, visualized by iodine). Following completion, the product was precipitated by adding water to the reaction solution. The resulting mixture was left for 6 hours before being vacuum filtered and a red solid was isolated. The crude product was redissolved in hot acetone and cooled in an ice water bath then vacuum filtered. A yellowish powder was afforded in $64 \%$ yield $(0.707 \mathrm{~g}) .{ }^{1} \mathrm{H}-\mathrm{NMR}\left(300 \mathrm{MHz}, \mathrm{DMSO}-\mathrm{d}^{6}\right) \delta: 7.98(\mathrm{~s}, 1 \mathrm{H})$, $6.45(\mathrm{~s}, 2 \mathrm{H}), 2.90(\mathrm{t}, 2 \mathrm{H}, \mathrm{J}=7.1 \mathrm{~Hz}), 2.17(\mathrm{t}, 2 \mathrm{H}, \mathrm{J}=8.4 \mathrm{~Hz}$ ), 1.62 (quintet, 2H, J=7.0), 1.46, $1.35(\mathrm{~m}, 4 \mathrm{H}), 1.21(\mathrm{~s}$, 10H). ${ }^{13} \mathrm{C}-\mathrm{NMR}\left(75 \mathrm{MHz}, \mathrm{CDCl}_{3}\right) \delta: 174.5,159.9,156.9$, (2 broad low intensity peaks), 140.1, 39.5, 38.10, 33.6, 28.8, 28.7, 28.5, 28.01, 27.7, 24.5. MS ((+) ESI): calc'd for $\mathrm{C}_{16} \mathrm{H}_{26} \mathrm{~N}_{5} \mathrm{O}_{2} \mathrm{~S}_{2}{ }^{+}=384.15 \mathrm{amu}$, obtained $=384.27 \mathrm{amu}$. 
<smiles>[R]C([R])C(=O)OCC(COC(=O)C([R])[R])NC(=O)CCCCCCCCCSSc1nc(N)nc2[nH]cnc12</smiles>

$$
\begin{array}{ll}
\text { MP-C14: } & R=\mathrm{C}_{12} \mathrm{H}_{25} ; \mathrm{R}^{\prime}=\mathrm{H} \\
\text { MP-C16: } & \mathrm{R}=\mathrm{C}_{14} \mathrm{H}_{29} ; \mathrm{R}^{\prime}=\mathrm{H} \\
\text { MP-dC16: } \mathrm{R}=\mathrm{C}_{8} \mathrm{H}_{17} ; \mathrm{R}^{\prime}=\mathrm{C}_{6} \mathrm{H}_{13} \\
\text { MP-C18: } \quad \mathrm{R}=\mathrm{C}_{16} \mathrm{H}_{33} ; \mathrm{R}^{\prime}=\mathrm{H} \\
\text { TG-C14: } \mathrm{R}=\mathrm{C}_{12} \mathrm{H}_{25} ; \mathrm{R}^{\prime}=\mathrm{H} \\
\text { TG-C16: } & \mathrm{R}=\mathrm{C}_{14} \mathrm{H}_{29} ; \mathrm{R}^{\prime}=\mathrm{H} \\
\text { TG-dC16: } & \mathrm{R}=\mathrm{C}_{8} \mathrm{H}_{17} ; \mathrm{R}^{\prime}=\mathrm{C}_{6} \mathrm{H}_{13}
\end{array}
$$

General Procedure: In a round bottom flask was dissolved 8 (1.1 equiv.) in dried DCM/DMF (1:1;10 mM). To this, HBTU (1.2 equiv.), 3(a-d) (1.0 equiv.), and triethylamine (2.0 equiv.) were then added and the resulting solution was left stirring at room temperature for 20 hours. When the reaction was complete, visualized by ${ }^{1} \mathrm{H}-$ $\mathrm{NMR}$, the reaction solution was diluted with EtOAc, and washed once with $1 \mathrm{M} \mathrm{HCl}$ and 3 times with $\mathrm{H}_{2} \mathrm{O}$. The organic layer was then dried over anhydrous sodium sulfate and then gravity filtered. DCM and EtOAc were removed by roto-evaporation. The crude product was purified by flash column chromatography on silica, using EtOAc/Hexanes as the eluent. Full characterization by ${ }^{13} \mathrm{C}$ NMR was hampered by low intensity signals from quaternary carbons in the purine in many cases.

MP-C14: By the general procedure: 8-MP (0.113 g, $0.308 \mathrm{mmol})$ in dried DCM/DMF (14.6 mL/ $14.6 \mathrm{~mL} ; 10 \mathrm{mM})$, HBTU (0.133 g, $0.352 \mathrm{mmol}, 1.20$ equiv.), $3 \mathrm{a}(0.150 \mathrm{~g}, 0.293 \mathrm{mmol})$, and triethylamine $(0.082 \mathrm{~mL}, 0.59 \mathrm{mmol})$; as a light yellow solid in a $28 \%$ yield $(0.072 \mathrm{~g}){ }^{1} \mathrm{H}-\mathrm{NMR}\left(300 \mathrm{MHz}, \mathrm{CDCl}_{3}\right) \delta: 11.75(\mathrm{~s}, 1 \mathrm{H}), 8.90(\mathrm{~s}, 1 \mathrm{H}), 8.22(\mathrm{~s}, 1 \mathrm{H})$, $6.02(\mathrm{~d}, 1 \mathrm{H}, \mathrm{J}=8.3 \mathrm{~Hz}) 4.52(\mathrm{~m}, 1 \mathrm{H}), 4.30(\mathrm{~m}, 2 \mathrm{H}), 4.11(\mathrm{~m}, 2 \mathrm{H}), 2.91(\mathrm{t}, 2 \mathrm{H}, \mathrm{J}=7.0 \mathrm{~Hz}), 2.32(\mathrm{t}, 4 \mathrm{H}, \mathrm{J}=7.9 \mathrm{~Hz}), 2.02(\mathrm{t}$, $2 \mathrm{H}, \mathrm{J}=7.9 \mathrm{~Hz}), 1.66(\mathrm{~m}, 6 \mathrm{H}), 1.26,1.12(\mathrm{~m}, 54 \mathrm{H}), 0.88(\mathrm{t}, 6 \mathrm{H}, \mathrm{J}=7.0)$. MS ((+) ESI): calc'd for $\mathrm{C}_{47} \mathrm{H}_{84} \mathrm{~N}_{5} \mathrm{O}_{5} \mathrm{~S}_{2}{ }^{+}=862.59$ amu, obtained $=862.53 \mathrm{amu}$.

MP-C16: By the general procedure: 8-MP $(0.0648 \mathrm{~g}, 0.176 \mathrm{mmol})$ in dried DCM/DMF $(9.0 \mathrm{~mL} / 9.0 \mathrm{~mL} ; 10 \mathrm{mM})$, HBTU $(0.112 \mathrm{~g}, 0.211 \mathrm{mmol}), 3 \mathrm{~b}(0.0648 \mathrm{~g}, 0.176 \mathrm{mmol})$, and triethylamine $(0.076 \mathrm{~mL}, 0.53 \mathrm{mmol})$; as a white solid in a $63 \%$ yield $(0.102 \mathrm{~g}){ }^{1} \mathrm{H}-\mathrm{NMR}\left(300 \mathrm{MHz}, \mathrm{CDCl}_{3}\right) \delta: 12.88(\mathrm{~s}, 1 \mathrm{H}), 8.91(\mathrm{~s}, 1 \mathrm{H}), 8.29(\mathrm{~s}, 1 \mathrm{H}), 6.05(\mathrm{~d}, 1 \mathrm{H}$, $\mathrm{J}=8.2 \mathrm{~Hz}), 4.50(\mathrm{~m}, 1 \mathrm{H}), 4.26(\mathrm{~m}, 2 \mathrm{H}), 4.09(\mathrm{~m}, 2 \mathrm{H}), 2.91(\mathrm{t}, 2 \mathrm{H}, 7.1 \mathrm{~Hz}), 2.30(\mathrm{t}, 4 \mathrm{H}, \mathrm{J}=7.6 \mathrm{~Hz}), 2.20(\mathrm{t}, 2 \mathrm{H}, \mathrm{J}=7.1$ $\mathrm{Hz}), 1.65(\mathrm{~m}, 6 \mathrm{H}), 1.24,1.17(\mathrm{~m}, 62 \mathrm{H}), 0.87(\mathrm{t}, 6 \mathrm{H}, \mathrm{J}=6.9 \mathrm{~Hz}) .{ }^{13} \mathrm{C}-\mathrm{NMR}\left(75 \mathrm{MHz}, \mathrm{CDCl}_{3}\right) \delta: 174.1,173.8,160.5$, 152.6, 149.9, 142.0, 131.4, 62.9, 48.0, 39.3, 36.9, 34.3, 32.1, 29.9, 29.9, 29.8, 29.7, 29.6, 29.5, 29.4, 29.1, 28.7, 28.3, 25.9, 25.1, 22.9, 14.3. MS ((+) ESI): calc'd for $\mathrm{C}_{51} \mathrm{H}_{92} \mathrm{~N}_{5} \mathrm{O}_{5} \mathrm{~S}_{2}{ }^{+}=918.65 \mathrm{amu}$, obtained $=918.60 \mathrm{amu}$.

MP-bC16: By the general procedure: 8-MP $(0.136 \mathrm{~g}, 0.370 \mathrm{mmol})$ in dried DCM/DMF $(17.5 \mathrm{~mL} / 17.5 \mathrm{~mL} ; 10 \mathrm{mM})$, HBTU $(0.160 \mathrm{~g}, 0.422 \mathrm{mmol}), 3 \mathrm{c}(0.200 \mathrm{~g}, 0.352 \mathrm{mmol})$, and triethylamine $(0.126 \mathrm{~mL}, 0.704 \mathrm{mmol})$; As a light yellow oil in a $42 \%$ yield $(0.137 \mathrm{~g}){ }^{1} \mathrm{H}-\mathrm{NMR}\left(300 \mathrm{MHz}, \mathrm{CDCl}_{3}\right) \delta: 12.8(\mathrm{~s}, 1 \mathrm{H}), 8.90(\mathrm{~s}, 1 \mathrm{H}), 8.29(\mathrm{~s}, 1 \mathrm{H}), 5.97(\mathrm{~d}, 1 \mathrm{H}$, $\mathrm{J}=8.8 \mathrm{~Hz}), 4.48(\mathrm{~m}, 1 \mathrm{H}), 4.26(\mathrm{~m}, 2 \mathrm{H}), 4.09(\mathrm{~m}, 2 \mathrm{H}), 2.90(\mathrm{t}, 2 \mathrm{H}, \mathrm{J}=7.3 \mathrm{~Hz}), 2.32(\mathrm{~m}, 2 \mathrm{H}), 2.2(\mathrm{~m}, 2 \mathrm{H}), 1.68,1.55$, $1.42(\mathrm{~m}, 14 \mathrm{H}), 1.22,1.16(\mathrm{~m}, 5 \mathrm{OH}), 0.85(\mathrm{t}, 12 \mathrm{H}, \mathrm{J}=6.9 \mathrm{~Hz}) .{ }^{13} \mathrm{C}\left(75 \mathrm{MHz}, \mathrm{CDCl}_{3}\right) \delta: 176.5,173.3,152.2,142.0,62.4$, 48.0, 45.6, 39.1, 36.7, 32.3, 32.0, 31.8, 31.6, 29.7, 29.5, 29.4, 29.2, 29.2, 28.9, 28.5, 28.2, 27.4, 27.4, 25.6, 22.6, 22.6, 14.0, 14.0. MS ((+) ESI): calc'd for $\mathrm{C}_{51} \mathrm{H}_{92} \mathrm{~N}_{5} \mathrm{O}_{5} \mathrm{~S}_{2}{ }^{+}=918.65 \mathrm{amu}$, obtained $=918.53 \mathrm{amu}$.

MP-C18: By the general procedure: 8-MP $(0.062 \mathrm{~g}, 0.168 \mathrm{mmol})$ in dried DCM/DMF $(8.0 \mathrm{~mL} / 8.0 \mathrm{~mL} ; 10 \mathrm{mM})$, HBTU $(0.073 \mathrm{~g}, 0.19 \mathrm{mmol}), 3 \mathrm{~d}(0.100 \mathrm{~g}, 0.160 \mathrm{mmol})$, and triethylamine $(0.045 \mathrm{~mL}, 0.32 \mathrm{mmol})$ ' as a white solid in a $32 \%$ yield $(0.050 \mathrm{~g}) .{ }^{1} \mathrm{H}-\mathrm{NMR}\left(300 \mathrm{MHz}, \mathrm{CDCl}_{3}\right) \delta: 8.91(\mathrm{~s}, 1 \mathrm{H}), 8.27(\mathrm{~s}, 1 \mathrm{H}), 6.01(\mathrm{~d}, 1 \mathrm{H}, \mathrm{J}=8.3 \mathrm{~Hz}), 4.50(\mathrm{~m}$, $1 \mathrm{H}), 4.26(\mathrm{~m}, 2 \mathrm{H}), 4.09(\mathrm{~m}, 2 \mathrm{H}), 2.90(\mathrm{t}, 2 \mathrm{H}, \mathrm{J}=7.2 \mathrm{~Hz}), 2.30(\mathrm{t}, 4 \mathrm{H}, \mathrm{J}=7.8 \mathrm{~Hz}), 2.20(\mathrm{t}, 2 \mathrm{H}, \mathrm{J}=7.8 \mathrm{~Hz}), 1.68,1.59(\mathrm{~m}$,

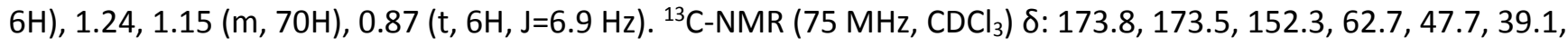


36.7, 34.1, 31.9, 29.7, 29.6, 29.5, 29.3, 29.2, 29.1, 29.1, 29.0, 28.9, 28.8, 28.4, 28.0, 25.6, 24.9, 22.7, 14.1. MS ((+) ESI): calc'd for $\mathrm{C}_{55} \mathrm{H}_{100} \mathrm{~N}_{5} \mathrm{O}_{5} \mathrm{~S}_{2}^{+}=974.72 \mathrm{amu}$, obtained $=974.67 \mathrm{amu}$.

TG-C14: By the general procedure: 8-TG $(0.118 \mathrm{~g}, 0.308 \mathrm{mmol})$ in dried DCM/DMF (14.6 mL/ $14.6 \mathrm{~mL} ; 10 \mathrm{mM})$, HBTU (0.133 g, $0.352 \mathrm{mmol}), 3 \mathrm{a}(0.150 \mathrm{~g}, 0.293 \mathrm{mmol})$, and triethylamine $(0.082 \mathrm{~mL}, 0.586 \mathrm{mmol})$; as a light yellow solid was afforded in $18 \%$ yield $(0.047 \mathrm{~g}){ }^{1} \mathrm{H}-\mathrm{NMR}\left(300 \mathrm{MHz}, \mathrm{CDCl}_{3}\right) \delta: 7.92(\mathrm{~s}, 1 \mathrm{H}), 6.10(\mathrm{~d}, 1 \mathrm{H}, \mathrm{J}=8.10 \mathrm{~Hz})$, $5.31(\mathrm{~s}, 2 \mathrm{H}), 4.52(\mathrm{~m}, 1 \mathrm{H}), 4.29(\mathrm{~m}, 2 \mathrm{H}), 4.12(\mathrm{~m}, 2 \mathrm{H}), 2.88(\mathrm{t}, 2 \mathrm{H}, \mathrm{J}=7.3 \mathrm{~Hz}), 2.32(\mathrm{t}, 4 \mathrm{H}, \mathrm{J}=7.3 \mathrm{~Hz}), 2.18(\mathrm{t}, 2 \mathrm{H}$, $\mathrm{J}=7.3 \mathrm{~Hz}), 1.68,1.59(\mathrm{~m}, 6 \mathrm{H}), 1.25,1.18(\mathrm{~m}, 54 \mathrm{H}), 0.88(\mathrm{t}, 6 \mathrm{H}, \mathrm{J}=6.9 \mathrm{~Hz}) .{ }^{13} \mathrm{C}-\mathrm{NMR}\left(75 \mathrm{MHz}, \mathrm{CDCl}_{3}\right) \delta: 174.1,173.7$, 159.7, 139.6, 63.0, 48.0, 39.4, 36.9, 34.3, 32.1, 29.9, 29.9, 29.8, 29.7, 29.6, 29.5, 29.4, 29.4, 29.3, 29.1, 28.7, 28.3, 25.8, 14.3. $\mathrm{MS}((+) \mathrm{ESI}):$ calc'd for $\mathrm{C}_{47} \mathrm{H}_{85} \mathrm{~N}_{6} \mathrm{O}_{5} \mathrm{~S}_{2}{ }^{+}=877.60 \mathrm{amu}$, obtained $=877.67 \mathrm{amu}$.

TG-C16: By the general procedure: 8-TG $(0.068 \mathrm{~g}, 0.18 \mathrm{mmol})$ in dried DCM/DMF $(8.8 \mathrm{~mL} / 8.8 \mathrm{~mL} ; 10 \mathrm{mM}), \mathrm{HBTU}$ $(0.112 \mathrm{~g}, 0.211 \mathrm{mmol}), 3 \mathrm{~b}(0.120 \mathrm{~g}, 0.18 \mathrm{mmol})$, and triethylamine $(0.076 \mathrm{~mL}, 0.53 \mathrm{mmol})$; as a light yellow solid in a $49 \%$ yield $(0.081 \mathrm{~g}) .{ }^{1} \mathrm{H}-\mathrm{NMR}\left(300 \mathrm{MHz}, \mathrm{CDCl}_{3}\right) \delta: 11.23(\mathrm{~s}, 1 \mathrm{H}), 7.87(\mathrm{~s}, 1 \mathrm{H}), 6.08(\mathrm{~d}, 1 \mathrm{H}, \mathrm{J}=8.4 \mathrm{~Hz}), 5.24(\mathrm{~s}$, $2 \mathrm{H}), 4.51(\mathrm{~m}, 1 \mathrm{H}), 4.29(\mathrm{~m}, 2 \mathrm{H}), 4.11(\mathrm{~m}, 2 \mathrm{H}), 2.87(\mathrm{t}, 2 \mathrm{H}, \mathrm{J}=7.0 \mathrm{~Hz}), 2.31(\mathrm{t}, 2 \mathrm{H}, \mathrm{J}=7.5 \mathrm{~Hz}), 2.17(\mathrm{t}, 2 \mathrm{H}, \mathrm{J}=7.6 \mathrm{~Hz})$, $1.68,1.60(\mathrm{~m}, 6 \mathrm{H}), 1.24,1.17(\mathrm{~m}, 62 \mathrm{H}), 0.87(\mathrm{t}, 6 \mathrm{H}, \mathrm{J}=7.0 \mathrm{~Hz}) .{ }^{13} \mathrm{C}-\mathrm{NMR}\left(75 \mathrm{MHz}, \mathrm{CDCl}_{3}\right): 173.9,173.5,159.4,62.8$, 47.7, 39.2, 36.6, 34.1, 31.9, 29.7, 29.6, 29.4, 29.3, 29.2, 29.1, 29.0, 29.0, 28.8, 28.4, 28.1, 25.6, 24.9, 22.7, 14.1. MS ((+) ESI): calc'd for $\mathrm{C}_{51} \mathrm{H}_{93} \mathrm{~N}_{6} \mathrm{O}_{5} \mathrm{~S}_{2}^{+}=933.66 \mathrm{amu}$, obtained $=933.73 \mathrm{amu}$.

TG-bC16: By the general procedure: 8-TG $(0.071 \mathrm{~g}, 0.19 \mathrm{mmol})$ in dried DCM/DMF $(8.8 \mathrm{~mL} / 8.8 \mathrm{~mL} ; 10 \mathrm{mM})$, HBTU $(0.080 \mathrm{~g}, 0.21), 3 \mathrm{c}(0.100 \mathrm{~g}, 0.176 \mathrm{mmol})$, and triethylamine $(0.049 \mathrm{~mL}, 0.35 \mathrm{mmol})$; as a light yellow liquid in a $37 \%$ yield $(0.061 \mathrm{~g}){ }^{1} \mathrm{H}-\mathrm{NMR}\left(300 \mathrm{MHz}, \mathrm{CDCl}_{3}\right) \delta: 7.88(\mathrm{~s}, 1 \mathrm{H}), 6.05(\mathrm{~d}, 1 \mathrm{H}, \mathrm{J}=8.2 \mathrm{~Hz}), 5.20(\mathrm{~s}, 2 \mathrm{H}), 4.50(\mathrm{~m}$, $1 \mathrm{H}), 4.30(\mathrm{~m}, 2 \mathrm{H}), 4.12(\mathrm{~m}, 2 \mathrm{H}), 2.87(\mathrm{t}, 2 \mathrm{H}, \mathrm{J}=7.1 \mathrm{~Hz}), 2.35(\mathrm{~m}, 2 \mathrm{H}), 2.15(\mathrm{t}, 2 \mathrm{H}, \mathrm{J}=7.4 \mathrm{~Hz}), 1.68,1.57,1.45(\mathrm{~m}$, $14 \mathrm{H}), 1.24,1.16(\mathrm{~m}, 50 \mathrm{H}) 0.86(\mathrm{t}, 12 \mathrm{H}, \mathrm{J}=6.5 \mathrm{~Hz}) .{ }^{13} \mathrm{C}-\mathrm{NMR}\left(75 \mathrm{MHz}, \mathrm{CDCl}_{3}\right)$ ס: 176.7, 173.3, 159.4, 62.5, 48.0, 45.7, 39.1, 36.6, 32.3, 31.8, 31.6, 29.7, 29.6, 29.4, 29.3, 29.2, 29.1,29.0, 29.0, 28.8, 28.4, 28.0, 27.4, 27.4, 25.5, 22.6, 22.6, 14.1, 14.00. MS ((+) ESI): calc'd for $\mathrm{C}_{51} \mathrm{H}_{93} \mathrm{~N}_{6} \mathrm{O}_{5} \mathrm{~S}_{2}{ }^{+}=933.66 \mathrm{amu}$, obtained $=933.60 \mathrm{amu}$. 


\section{Characterization of NHS-PEG:}

PEG-lipids have a high sensitivity to ESI-MS since the ethylene chains possess a high affinity towards positively charged species such as protons, potassium or sodium ions. Information obtained from the ESI-MS spectra allowed for the determination of the degree of polymerization $(n)$ in the starting sample (obtained from SigmaAldrich; $\mathrm{n} \sim 45$ ).

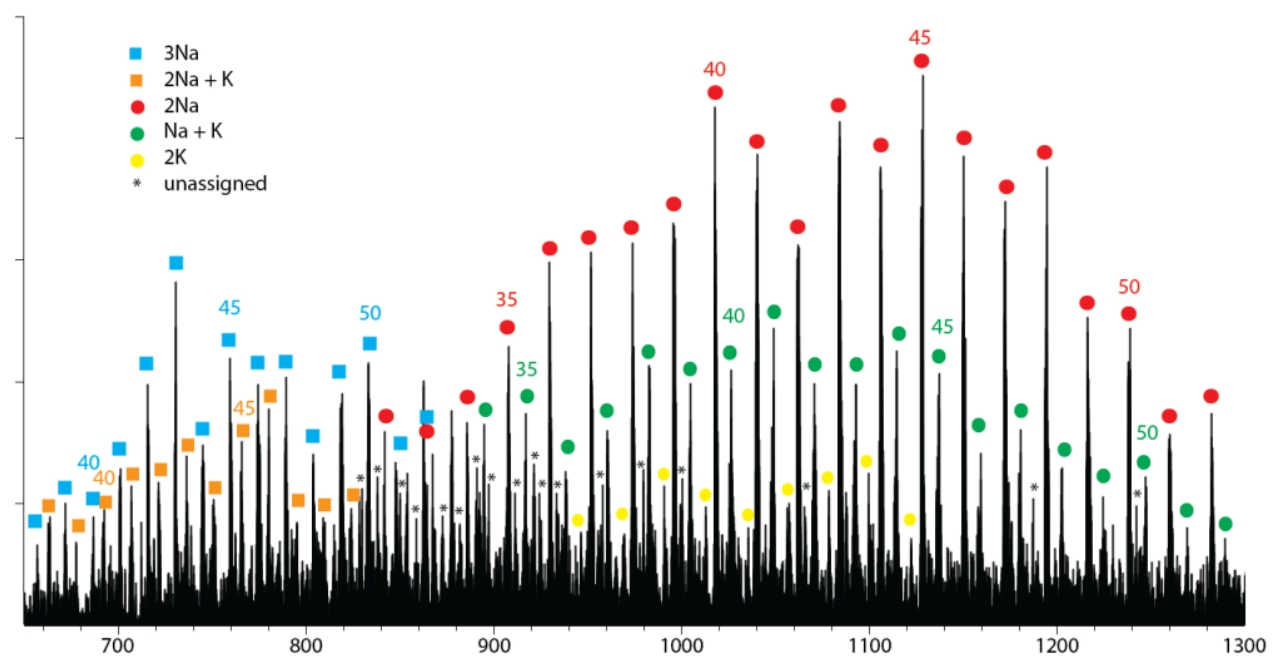

Figure S1: Mass spectrum generated from ESI-MS of the starting material NHS-PEG. The sample was treated with $0.1 \%$ TFA and $0.1 \% \mathrm{NaCl}$.

ESI-MS of the NHS-PEG starting material shows five different series for multiply-charged species (Figure S1): 1, $[\mathrm{M}+3 \mathrm{Na}+\mathrm{K}]^{3+}$ (orange squares); $2,[\mathrm{M}+3 \mathrm{Na}]^{3+}$ (blue squares); $3,[\mathrm{M}+2 \mathrm{Na}]^{2+}$ (red dots); $4,[\mathrm{M}+2 \mathrm{~K}]^{2+}$ (yellow dots); $5,[\mathrm{M}+\mathrm{Na}+\mathrm{K}]^{2+}$ (green dots). Series were identified by spacing (14.7 amu for triply charged; 22 amu for doubly charged). Figure S1 identifies 74 of the 87 ion clusters observed. Once the peaks were assigned, the intensities for a given $\mathrm{n}$ can then be summed, and the summed intensities plotted versus $\mathrm{n}$ with the data fit to a Gaussian with a high reliability (Figure S2). The experimental $n=43$ by this method.

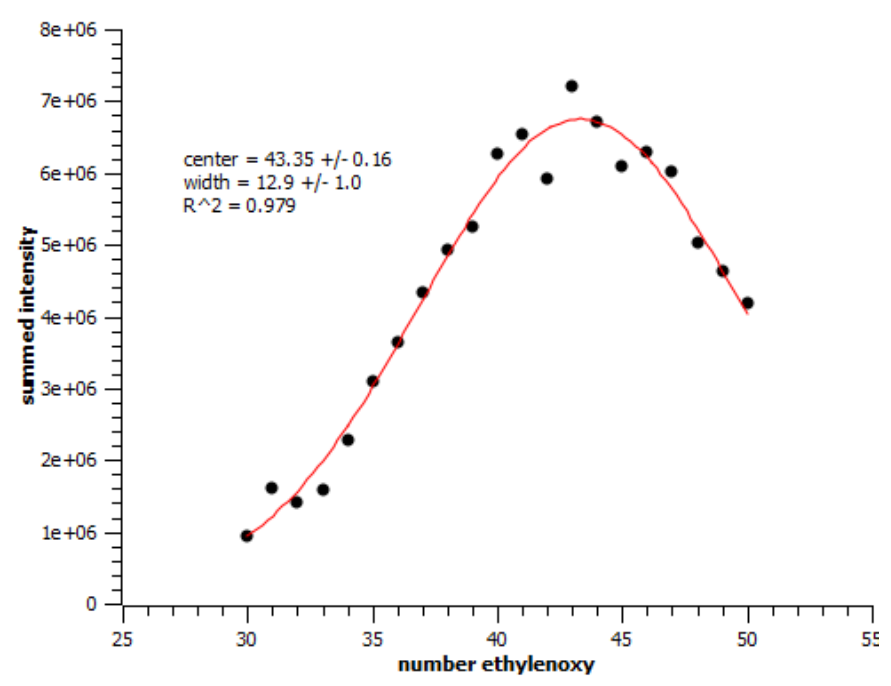

Figure S2: The intensities of a given $\mathrm{n}$ were summed and plotted versus $n$ for the NHS-PEG. The plot was then fitted to a Gaussian distribution. 
The ${ }^{1} \mathrm{H}-\mathrm{NMR}$ of the NHS-PEG (Figure S3) suggested that the starting material contained impurities. The expected integration of the ethylene protons should be $\sim 174 \mathrm{H}$; however, the observed integration was $297 \mathrm{H}$ indicating that there may be polymeric impurities present. Although there were unassigned peaks in the ESI-MS (Figure S1), these peaks were not found to correspond to a polymeric series. The singlet for the terminal methoxy shows at $3.39 \mathrm{ppm}$ with an integration of $5.2 \mathrm{H}$ which is considerably greater than the expected $3 \mathrm{H}$. Based on the integrations it is most likely that both ends terminate with methoxy groups - suggesting an MeO-PEG-OMe like structure. Assuming this is a single impurity, purity calculations based on the ethylene proton signal and methoxy proton signal give mol \% purities of $58 \%$ and $63 \%$, respectively. Averaging of these two approximations gives $61 \%$ purity for the NHS-PEG starting material.

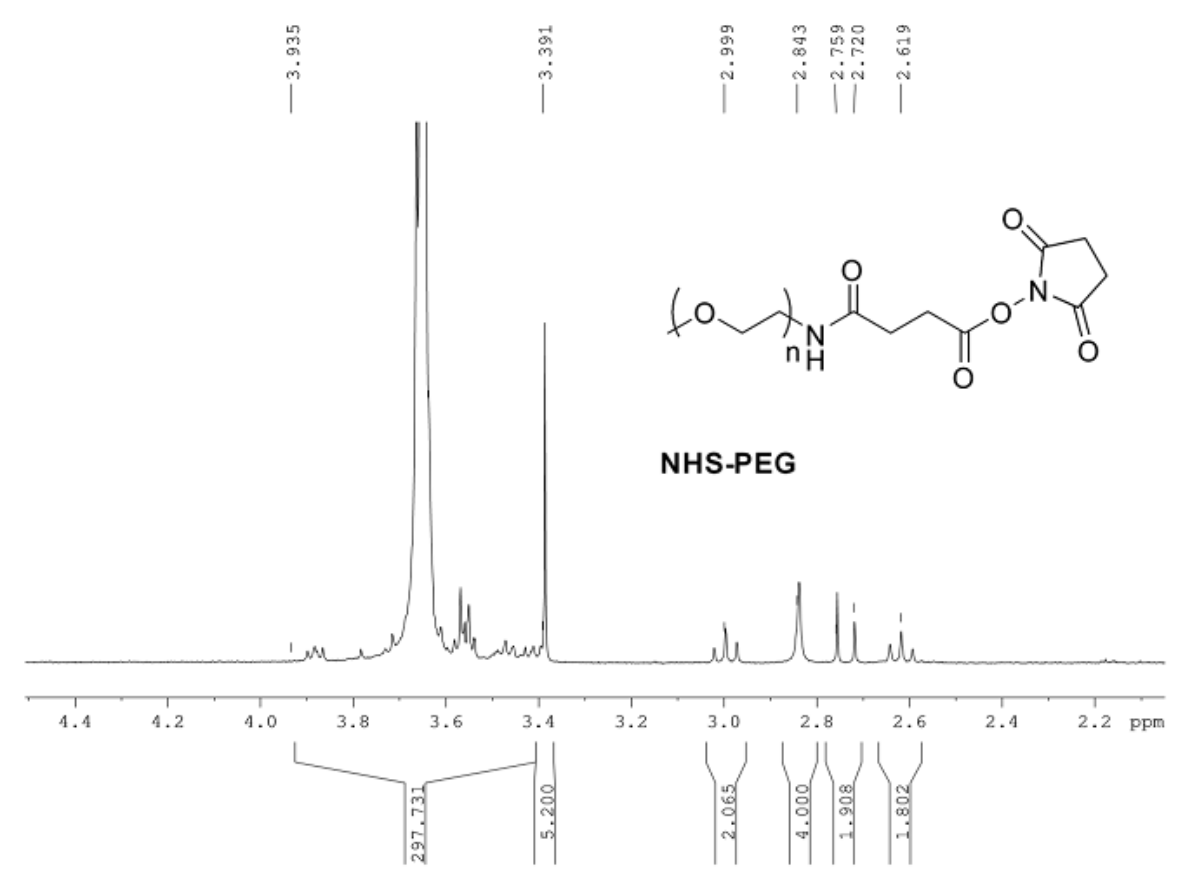

Figure S3: The integration of the methylene protons in the ${ }^{1} \mathrm{H}$ NMR $(300 \mathrm{MHz})$ of NHS-PEG, in $\mathrm{CDCl}_{3}$, was significantly different than the expected values.

\section{Synthesis of PEG-Lipids:}

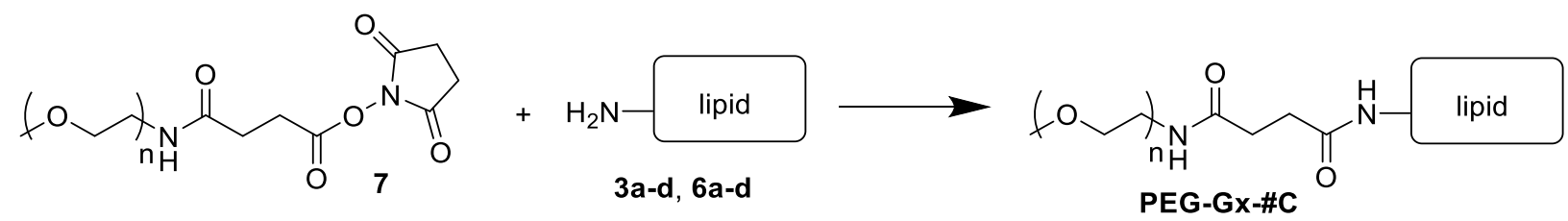

Scheme S3: Amide formation between PEG-NHS and the free primary amine on the synthetic lipids

General Procedure: An equimolar mixture of 7 and the amino-lipid was made to a concentration of $0.12 \mathrm{M}$ in pyridine in a vial. The vial was then sealed, the solution heated to $55^{\circ} \mathrm{C}$ and stirred for 48 hours. Following completion, as monitored by TLC (silica gel, $\mathrm{MeOH} / \mathrm{DCM}$ as eluent, visualised by iodine), the pyridine was removed on a roto-evaporator and the resulting mixture was purified by flash column chromatography on silica gel, using $\mathrm{MeOH} / \mathrm{DCM}$ as the eluent. 
PEG-G1-C14: By the general procedure: $7(0.111 \mathrm{~g}, 0.0488 \mathrm{mmol})$ and $3 \mathbf{a}(0.025 \mathrm{~g}, 0.049 \mathrm{mmol})$; as a white solid in a 70\% yield $(0.087 \mathrm{~g}) .{ }^{1} \mathrm{H}-\mathrm{NMR}\left(300 \mathrm{MHz}, \mathrm{CDCl}_{3}\right) \delta: 4.41(\mathrm{~m}, 1 \mathrm{H}), 4.16(\mathrm{~m}, 2 \mathrm{H}), 4.07(\mathrm{~m}, 2 \mathrm{H}), 3.40-3.90(\mathrm{~m}$, $174 \mathrm{H}), 3.38(\mathrm{~s}, 3 \mathrm{H}), 2.51(\mathrm{~s}, 4 \mathrm{H}), 2.31(\mathrm{t}, 4 \mathrm{H}, 7.8 \mathrm{~Hz}), 1.59(\mathrm{~m}, 4 \mathrm{H}), 1.24(\mathrm{~s}, 40 \mathrm{H}), 0.87(\mathrm{t}, 6 \mathrm{H}, \mathrm{J}=7.0 \mathrm{~Hz})$. ESI-MS see Figure S4.

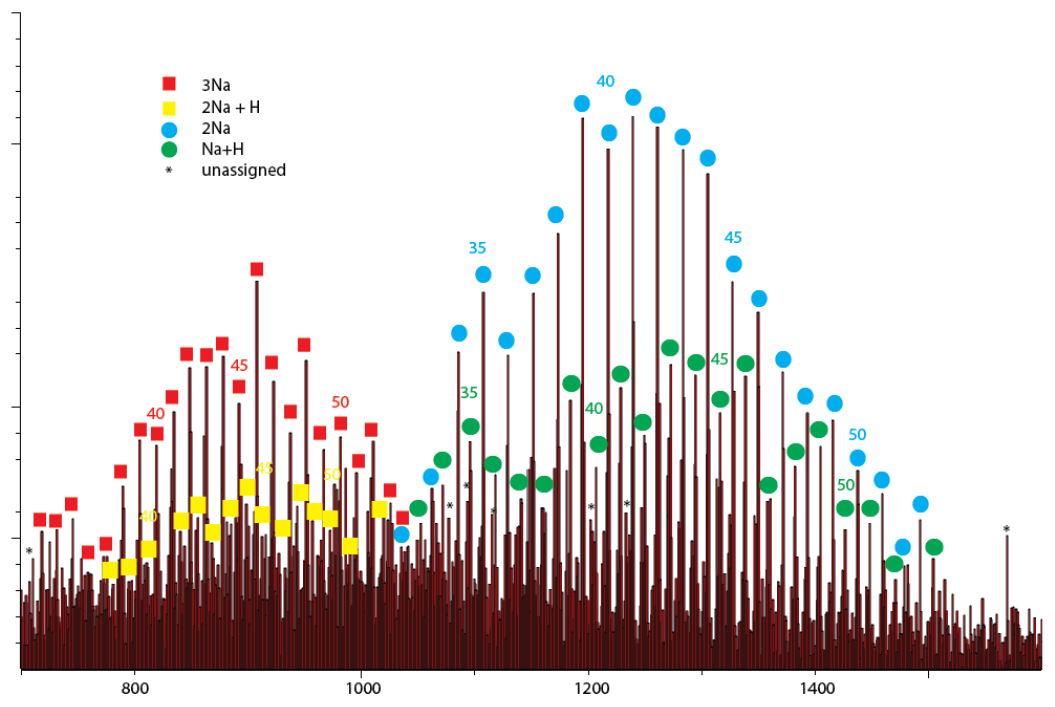

Figure S4: Mass spectrum generated from ESI-MS of PEG-G $\mathbf{H}_{1}$-C14. Sample was treated with $0.1 \%$ TFA and $0.1 \% \mathrm{NaCl}$. The compound shows $[\mathrm{M}+3 \mathrm{Na}]^{3+}$,

$[\mathrm{M}+2 \mathrm{Na}+\mathrm{H}]^{3+},[\mathrm{M}+2 \mathrm{Na}]^{2+}$, and $[\mathrm{M}+\mathrm{Na}+\mathrm{H}]^{2+}$ ion series where 78 of the 85 observed ion clusters are assigned.

PEG-G1-C16: By the general procedure: 7 (0.100 g, $0.0440 \mathrm{mmol})$ and $3 \mathbf{b}(0.025 \mathrm{~g}, 0.44 \mathrm{mmol})$; as a white solid in a $66 \%$ yield $(0.076 \mathrm{~g}) .{ }^{1} \mathrm{H}-\mathrm{NMR}\left(300 \mathrm{MHz}, \mathrm{CDCl}_{3}\right) \delta: 6.66(\mathrm{~d}, 1 \mathrm{H}, \mathrm{J}=8.3 \mathrm{~Hz}), 6.53(\mathrm{~m}, 1 \mathrm{H}), 4.40(\mathrm{~m}, 1 \mathrm{H}), 4.16(\mathrm{~m}$, $2 \mathrm{H}), 4.07(\mathrm{~m}, 2 \mathrm{H}), 3.38-3.90(\mathrm{~m}, 174 \mathrm{H}), 3.37(\mathrm{~s}, 3 \mathrm{H}), 2.51(\mathrm{~s}, 4 \mathrm{H}), 2.31(\mathrm{t}, 4 \mathrm{H}, \mathrm{J}=7.5 \mathrm{~Hz}), 1.59(\mathrm{~m}, 4 \mathrm{H}), 1.25(\mathrm{~s}, 48 \mathrm{H})$, $0.87(\mathrm{t}, 6 \mathrm{H}, \mathrm{J}=6.8 \mathrm{~Hz})$. ESI-MS see Figure $\mathrm{S} 5$

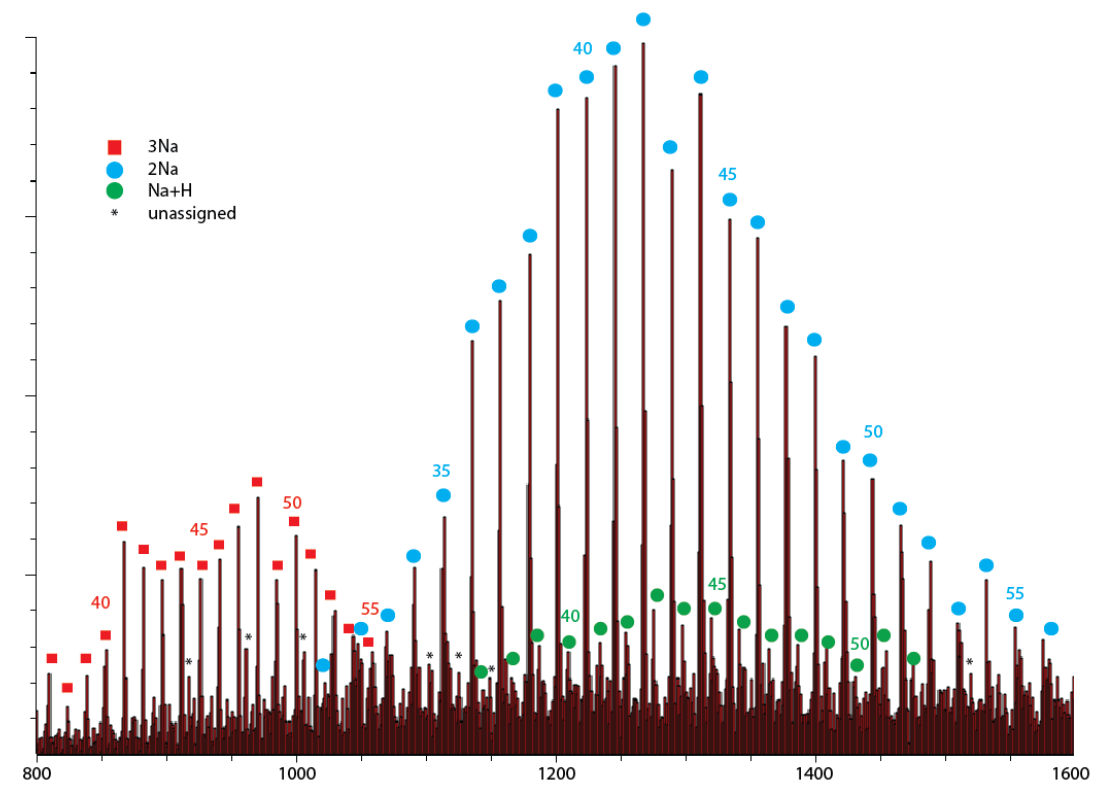

Figure S5: Mass spectrum generated from ESI-MS of PEG-G $\mathbf{G}_{1}-\mathbf{C 1 6}$. Sample was treated with $0.1 \%$ TFA and $0.1 \%$ $\mathrm{NaCl}$. The compound shows $[\mathrm{M}+3 \mathrm{Na}]^{3+}$, $[\mathrm{M}+2 \mathrm{Na}]^{2+}$, and $[\mathrm{M}+\mathrm{Na}+\mathrm{H}]^{2+}$ ion series where 60 of the 67 observed ion clusters are assigned. 
PEG-G1-bC16: By the general procedure: $7(0.100 \mathrm{~g}, 0.0440 \mathrm{mmol})$ and $3 \mathrm{c}(0.025 \mathrm{~g}, 0.044 \mathrm{mmol})$. A gel-like colorless solid was isolated in a $68 \%$ yield $(0.087 \mathrm{~g}) .{ }^{1} \mathrm{H}-\mathrm{NMR}\left(300 \mathrm{MHz}, \mathrm{CDCl}_{3}\right) \delta: 4.40(\mathrm{~m}, 1 \mathrm{H}), 4.19(\mathrm{~m}, 2 \mathrm{H}), 4.04$ $(\mathrm{m}, 2 \mathrm{H}), 3.39-3.90(\mathrm{~m}, 174), 3.38(\mathrm{~s}, 3 \mathrm{H}), 2.51(\mathrm{~s}, 4 \mathrm{H}), 2.34(\mathrm{~m}, 2 \mathrm{H}), 1.57,1.45(\mathrm{~m}, 8 \mathrm{H}), 1.25(\mathrm{~s}, 40 \mathrm{H}), 0.87(\mathrm{t}, 12 \mathrm{H}$, $\mathrm{J}=7.2 \mathrm{~Hz}$ ). ESI-MS see Figure $\mathrm{S6}$.

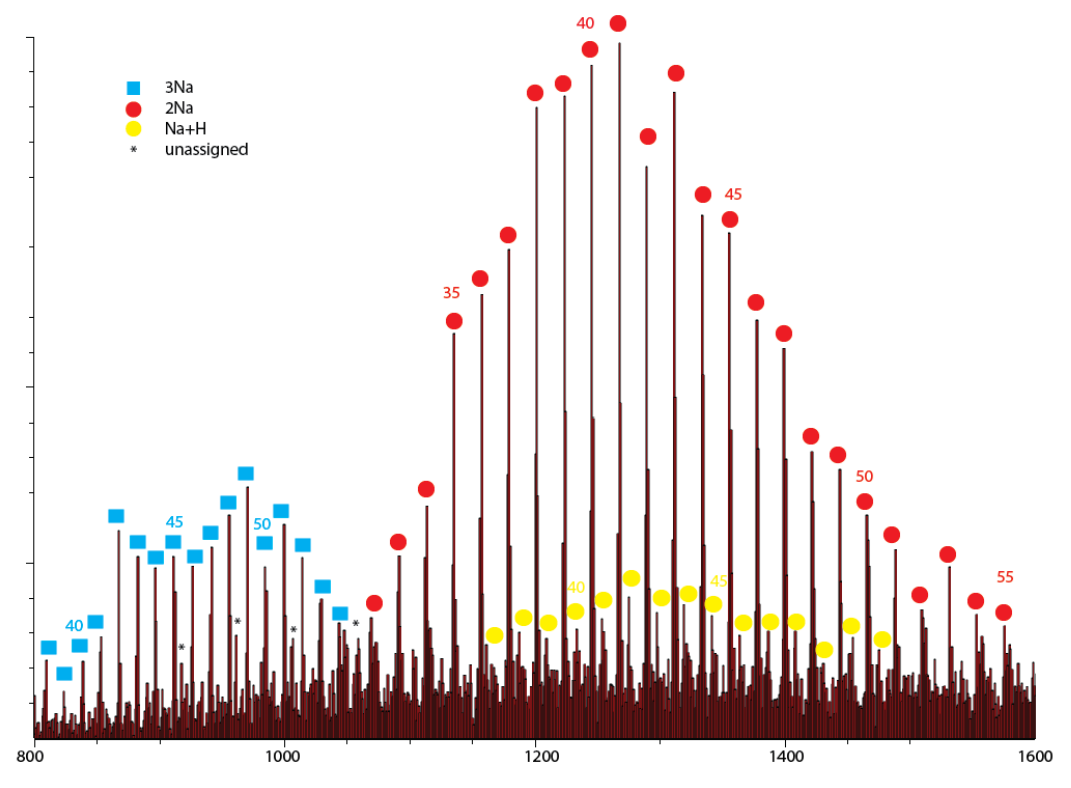

Figure S6: Mass spectrum generated from ESI-MS of PEG-G1-bC16. Sample was treated with $0.1 \%$ TFA and $0.1 \%$ $\mathrm{NaCl}$. The compound shows $[\mathrm{M}+3 \mathrm{Na}]^{3+}$, $[\mathrm{M}+2 \mathrm{Na}]^{2+}$, and $[\mathrm{M}+\mathrm{Na}+\mathrm{H}]^{2+}$ ion series where 56 of the 60 ion clusters are assigned.

PEG-G1-C18: By the general procedure: 7 (0.091 $\mathrm{g} 0.040 \mathrm{mmol})$ and $3 \mathbf{d}(0.025 \mathrm{~g}, 0.040 \mathrm{mmol})$; as a white solid in a $67 \%$ yield $(0.071 \mathrm{~g}) .{ }^{1} \mathrm{H}-\mathrm{NMR}\left(300 \mathrm{MHz}, \mathrm{CDCl}_{3}\right) \delta: 6.69(\mathrm{~d}, 1 \mathrm{H}, \mathrm{J}=8.9 \mathrm{~Hz}), 6.61(\mathrm{~m}, 1 \mathrm{H}), 4.41(\mathrm{~m}, 1 \mathrm{H}),, 4.16(\mathrm{~m}$, $2 \mathrm{H}), 4.07(\mathrm{~m}, 2 \mathrm{H}), 3.38-3.90(\mathrm{~m}, 174 \mathrm{H}), 3.37(\mathrm{~s}, 3 \mathrm{H}), 2.51(\mathrm{~s}, 4 \mathrm{H}), 2.31(\mathrm{t}, 4 \mathrm{H}, \mathrm{J}=7.8 \mathrm{~Hz}), 1.61(\mathrm{~m}, 4 \mathrm{H}), 1.24(\mathrm{~s}, 56 \mathrm{H})$, $0.87(\mathrm{t}, 6 \mathrm{H}, \mathrm{J}=7 \mathrm{~Hz})$. ESI-MS see Figure $\mathrm{S7}$.

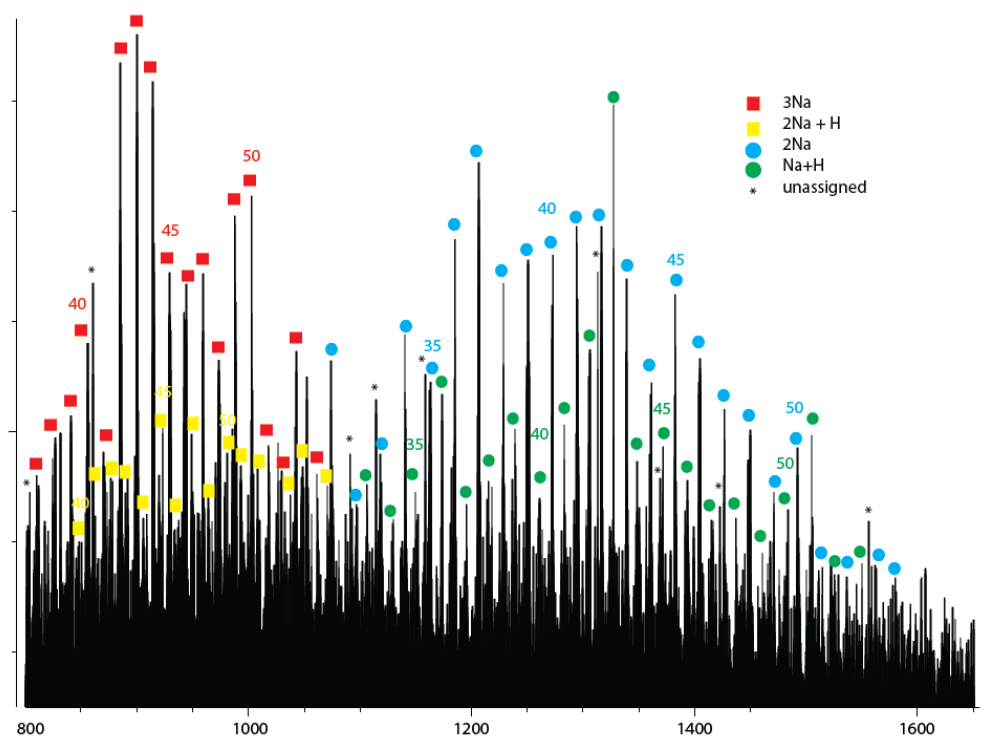

Figure S7: Mass spectrum generated from ESIMS of PEG-G1-C18. Sample was treated with $0.1 \%$ TFA and $0.1 \% \mathrm{NaCl}$. The compound shows $[\mathrm{M}+3 \mathrm{Na}]^{3+},[\mathrm{M}+2 \mathrm{Na}+\mathrm{H}]^{3+},[\mathrm{M}+2 \mathrm{Na}]^{2+}$, and $[\mathrm{M}+\mathrm{Na}+\mathrm{H}]^{2+}$ ion series where 79 of the 87 observed ion clusters are assigned. 
PEG-G2-C14: By the general procedure: $7(0.098 \mathrm{~g}, 0.043 \mathrm{mmol})$ and crude $6 \mathrm{a}(0.051 \mathrm{~g}, 0.043 \mathrm{mmol})$; as a white solid in a $52 \%$ yield $(0.072 \mathrm{~g}) .{ }^{1} \mathrm{H}-\mathrm{NMR}\left(300 \mathrm{MHz}, \mathrm{CDCl}_{3}\right) \delta: 4.39(\mathrm{~m}, 2 \mathrm{H})$ 3.89-4.26 $(\mathrm{m}, 14 \mathrm{H}), 3.37-3.90(\mathrm{~m}, 174 \mathrm{H})$, $3.36(\mathrm{~s}, 3 \mathrm{H}), 2.52(\mathrm{~m}, 4 \mathrm{H}), 2.31(\mathrm{t}, 4 \mathrm{H}, \mathrm{J}=7.6 \mathrm{~Hz}), 2.29(\mathrm{t}, 4 \mathrm{H}, \mathrm{J}=7.6 \mathrm{~Hz}), 1.24(\mathrm{~s}, 80 \mathrm{H}), 0.86(\mathrm{t}, 12 \mathrm{H}, 6.8 \mathrm{~Hz})$. ESI-MS see Figure $\mathrm{S} 8$.

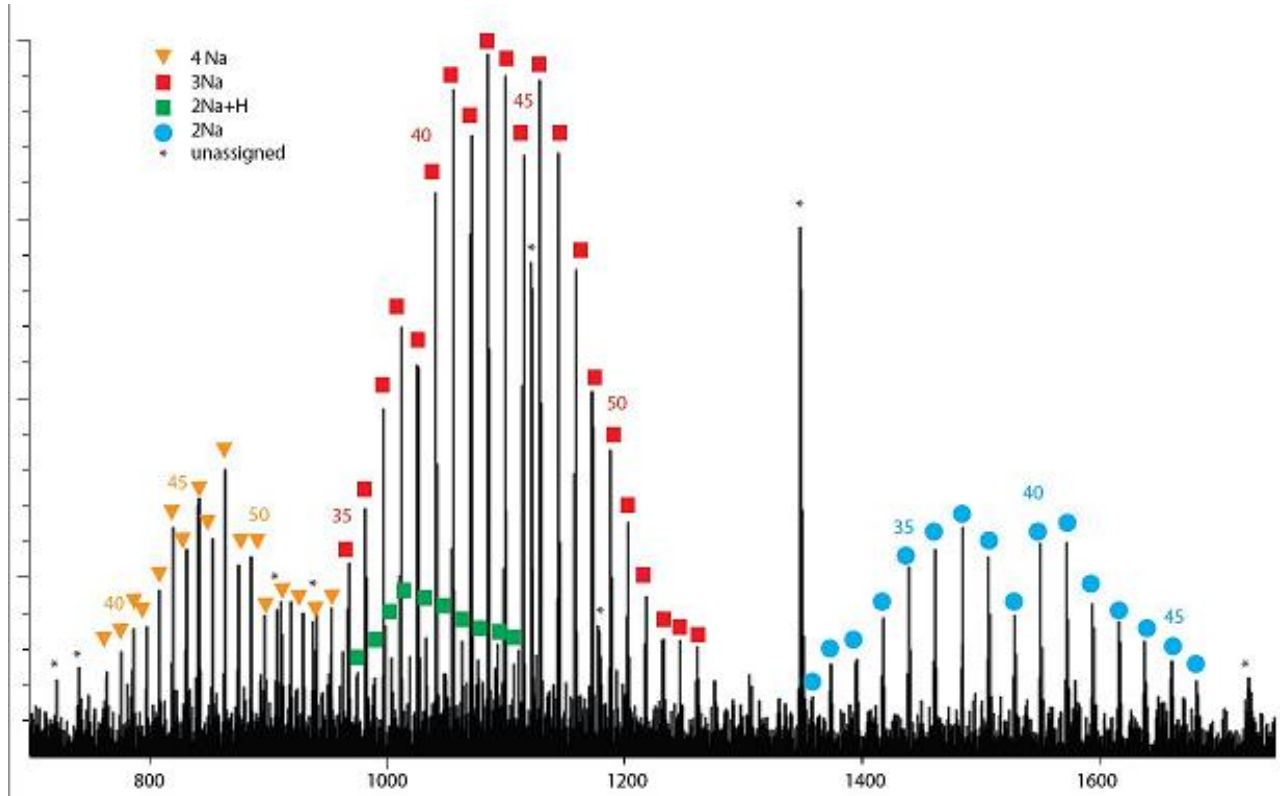

Figure S8: Mass spectrum generated from ESI-MS of PEG-G2-C14. Sample was treated with $0.1 \%$ TFA and $0.1 \% \mathrm{NaCl}$. The compound shows $[\mathrm{M}+4 \mathrm{Na}]^{4+}$, $[\mathrm{M}+3 \mathrm{Na}]^{3+},[\mathrm{M}+2 \mathrm{Na}+\mathrm{H}]^{3+}$, and $[\mathrm{M}+2 \mathrm{Na}]^{2+}$ ion series where 64 of the 71 observed ion clusters are assigned.

PEG-G2-C16: By the general procedure: 7 (0.123 g, $0.054 \mathrm{mmol})$ and crude $6 \mathrm{~b}(0.070 \mathrm{~g}, 0.054 \mathrm{mmol})$; as a white solid in $54 \%$ yield $(0.096 \mathrm{~g}){ }^{1} \mathrm{H}-\mathrm{NMR}\left(300 \mathrm{MHz}, \mathrm{CDCl}_{3}\right) \delta: 4.38(\mathrm{~m}, 2 \mathrm{H}), 3.88-4.26(\mathrm{~m}, 14 \mathrm{H}), 3.37-3.87(\mathrm{~m}, 174 \mathrm{H})$, $3.36(\mathrm{~s}, 3 \mathrm{H}), 2.53(\mathrm{~m}, 4 \mathrm{H}), 2.31(\mathrm{t}, 4 \mathrm{H}, \mathrm{J}=7.6 \mathrm{~Hz}), 2.29(\mathrm{t}, 4 \mathrm{H}, \mathrm{J}=7.6 \mathrm{~Hz}), 1.24(\mathrm{~s}, 96 \mathrm{H}), 0.86(\mathrm{t}, 12 \mathrm{H}, \mathrm{J}=7.0 \mathrm{~Hz})$. ESIMS see Figure $\mathrm{S9}$. 


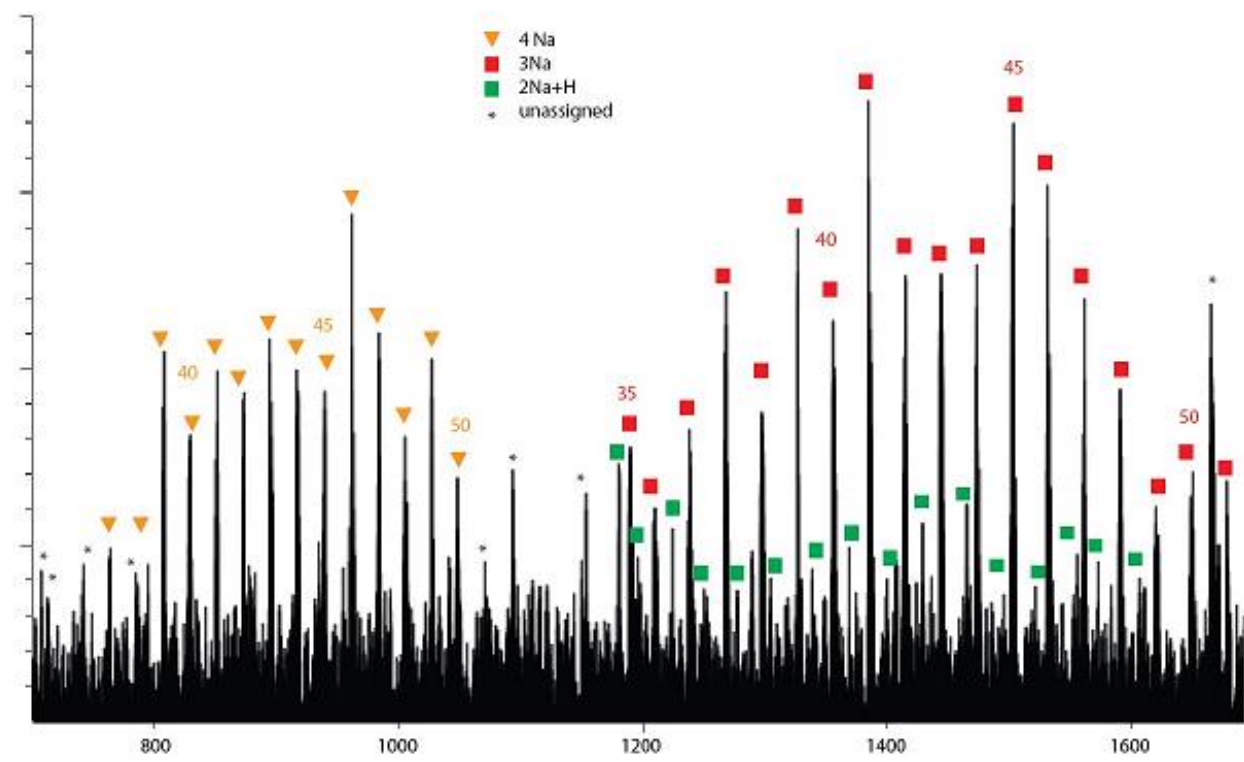

Figure S9: Mass spectrum generated from ESI-MS of PEG-G2-C16. Sample was treated with $0.1 \%$ TFA and $0.1 \% \mathrm{NaCl}$. The compound shows $[\mathrm{M}+4 \mathrm{Na}]^{4+},[\mathrm{M}+3 \mathrm{Na}]^{3+}$, and $[\mathrm{M}+2 \mathrm{Na}+\mathrm{H}]^{3+}$ ion series where 48 of the 56 observed ion clusters are assigned.

PEG-G2-bC16: By the general procedure: $7(0.130 \mathrm{~g}, 0.0572 \mathrm{mmol})$ and crude $6 \mathrm{c}(0.074 \mathrm{~g}, 0.057 \mathrm{mmol})$; as a white solid in 33\% yield (0.061 g). ${ }^{1} \mathrm{H}-\mathrm{NMR}\left(300 \mathrm{MHz}, \mathrm{CDCl}_{3}\right) \delta: 6.74(\mathrm{~s}, 1 \mathrm{H}), 6.66(\mathrm{~d}, 1 \mathrm{H}, \mathrm{J}=7.5 \mathrm{H}), 6.47(\mathrm{~s}, 1 \mathrm{H})$, $4.40(\mathrm{~m}, 2 \mathrm{H}), 3.87-4.22(\mathrm{~m}, 14 \mathrm{H}), 3.36-3.87(\mathrm{~m}, 174 \mathrm{H}), 3.35(\mathrm{~s}, 3 \mathrm{H}), 2.51(\mathrm{~m}, 4 \mathrm{H}), 2.31(\mathrm{~m}, 4 \mathrm{H}), 1.42,1.53(\mathrm{~m}$, $16 \mathrm{H}), 1.22(\mathrm{~s}, 80 \mathrm{H}), 0.85(\mathrm{t}, 24 \mathrm{H}, \mathrm{J}=7.0 \mathrm{~Hz})$. ESI-MS see Figure $\mathrm{S} 10$.

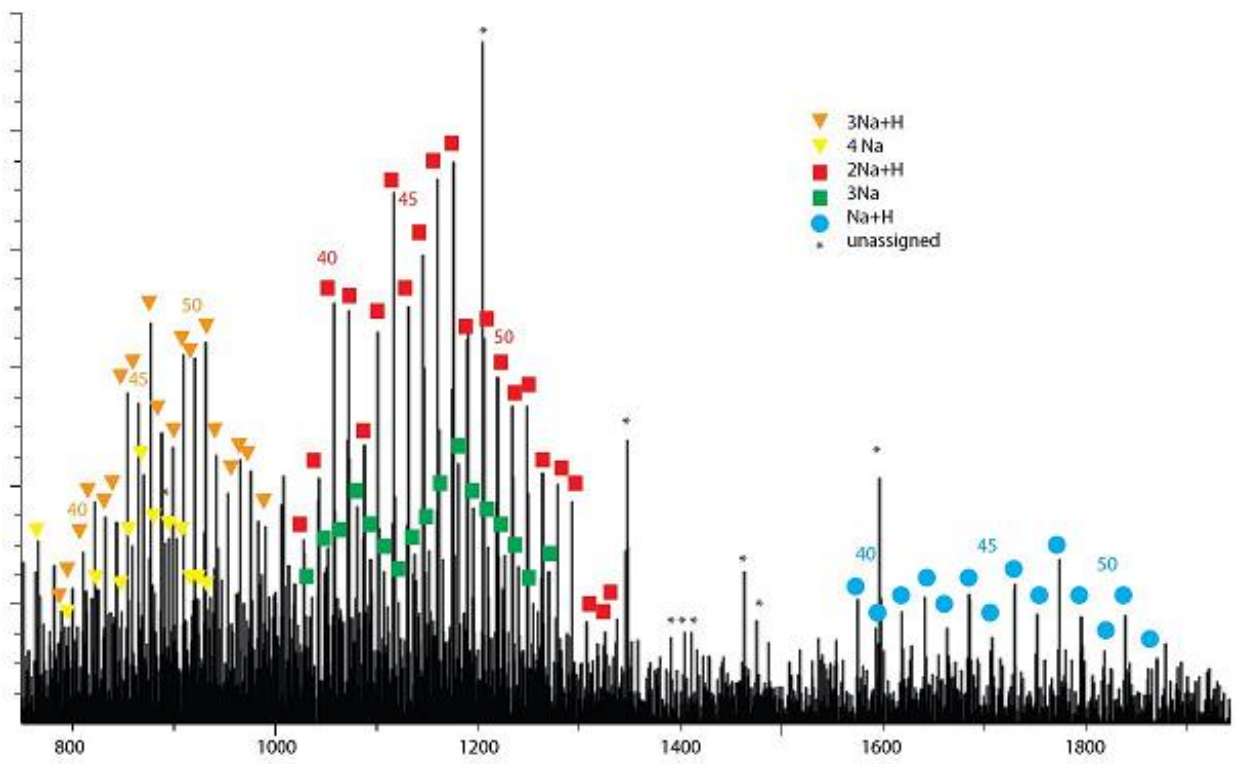

Figure S10: Mass spectrum generated from ESI-MS OF PEG-G2-bC16. Sample was treated with $0.1 \%$ TFA and $0.1 \% \mathrm{NaCl}$. The compound shows $[\mathrm{M}+4 \mathrm{Na}]^{4+}$, $[\mathrm{M}+3 \mathrm{Na}+\mathrm{H}]^{4+},[\mathrm{M}+3 \mathrm{Na}]^{3+}$, $[\mathrm{M}+2 \mathrm{Na}+\mathrm{H}]^{3+}$, and $[\mathrm{M}+\mathrm{Na}+\mathrm{H}]^{2+}$ ion series where 83 of the 92 observed ion clusters are assigned.

PEG-G2-C18: By the general procedure: $7(0.065 \mathrm{~g}, 0.029 \mathrm{mmol})$ and crude $6 \mathrm{~d}(0.040 \mathrm{~g}, 0.029 \mathrm{mmol}, 1.0$ equiv.); as a gel-like solid in $47 \%$ yield $(0.047 \mathrm{~g}) .{ }^{1} \mathrm{H}-\mathrm{NMR}\left(300 \mathrm{MHz}, \mathrm{CDCl}_{3}\right) \delta: 4.39(\mathrm{~m}, 2 \mathrm{H}), 3.89-4.27(\mathrm{~m}, 14 \mathrm{H}), 3.38-3.89$ $(\mathrm{m}, 174 \mathrm{H}), 3.37(\mathrm{~s}, 3 \mathrm{H}), 2.53(\mathrm{~m}, 4 \mathrm{H}), 2.32(\mathrm{t}, 4 \mathrm{H}, \mathrm{J}=7.4 \mathrm{~Hz}), 2.29(\mathrm{t}, 4 \mathrm{H}, \mathrm{J}=7.4 \mathrm{~Hz}), 1.24(\mathrm{~s}, 112 \mathrm{H}), 0.87(\mathrm{t}, 12 \mathrm{H}$, $\mathrm{J}=7.0 \mathrm{~Hz})$. ESI-MS see Figure S11. 


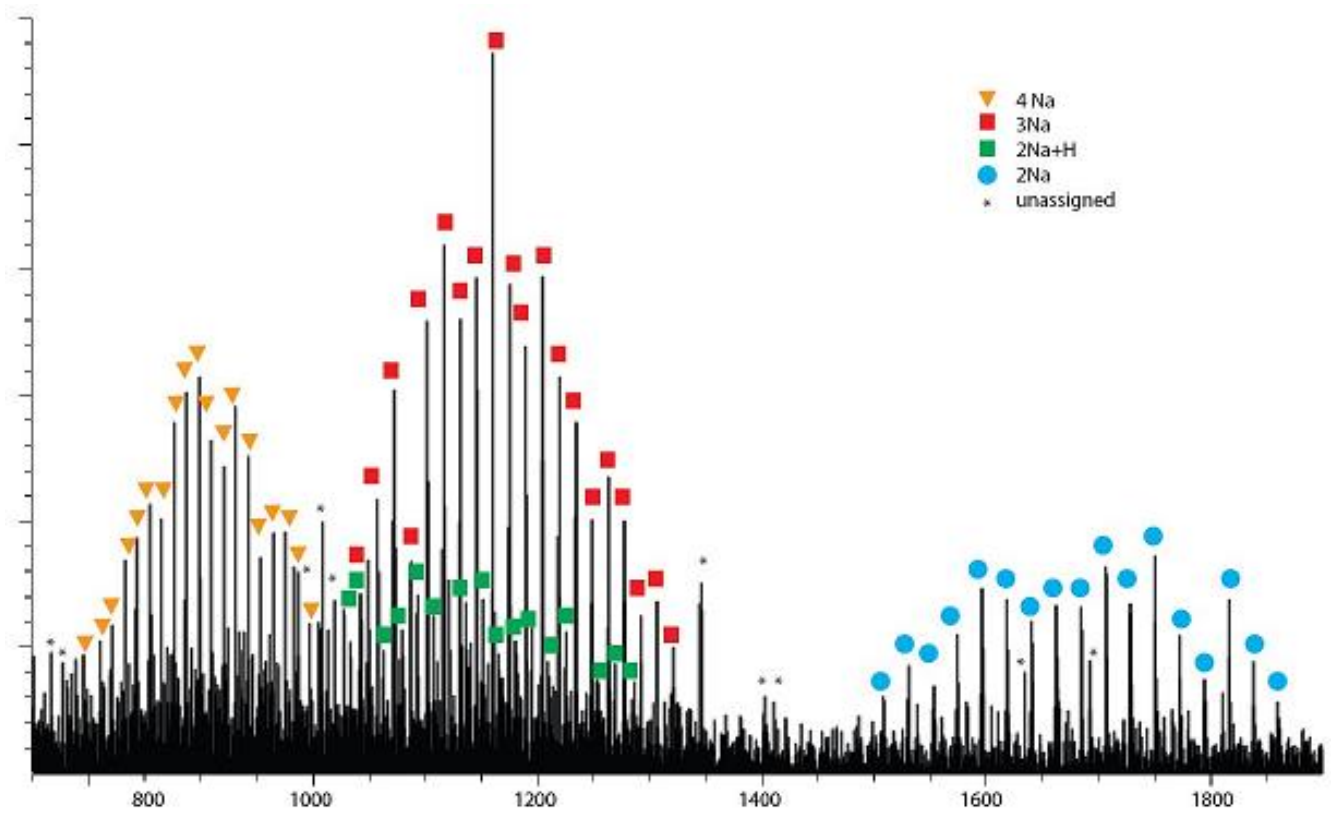

Figure S11: Mass spectrum generated from ESI-MS of PEG-G2C18. Sample was treated with $0.1 \%$ TFA and $0.1 \%$ $\mathrm{NaCl}$. The compound shows $[\mathrm{M}+4 \mathrm{Na}]^{4+}$, $[\mathrm{M}+3 \mathrm{Na}]^{3+}$, $[\mathrm{M}+2 \mathrm{Na}+\mathrm{H}]^{3+}$, and $[\mathrm{M}+2 \mathrm{Na}]^{2+}$ ion series where 73 of the 83 observed ion clusters ware assigned.

Table SO Summary of Gaussian fitting to the sum of intensities observed for a given $\mathrm{N}$

\begin{tabular}{clrr} 
& \multicolumn{1}{l}{ center } & \multicolumn{1}{l}{ width } & \multicolumn{1}{l}{$\mathrm{R}^{2}$} \\
$\mathbf{7}$ & $43.5 \pm 0.12$ & $14 \pm 1.5$ & 0.9796 \\
PEG-G1-C14 & $43.0 \pm 0.22$ & $24 \pm 15$ & 0.912 \\
PEG-G1-C16 & $43.6 \pm 0.27$ & $17 \pm 4.5$ & 0.9068 \\
PEG-G1-brC16 & $43.1 \pm 0.38$ & $14 \pm 3.9$ & 0.829 \\
PEG-G1-C18 & $43.7 \pm 0.17$ & $12 \pm 1.1$ & 0.9642 \\
PEG-G2-C14 & $43.1 \pm 0.14$ & $11 \pm 1.7$ & 0.969 \\
PEG-G2-C16 & $43.0 \pm 0.17$ & $9.0 \pm 1.3$ & 0.9517 \\
PEG-G2-brC16 & $46.5 \pm 0.8$ & $17 \pm 16$ & 0.6933 \\
PEG-G2-C18 & $43.0 \pm 0.24$ & $9.5 \pm 2.1$ & 0.9081
\end{tabular}

\section{Preparation of LNPS}

LNPs were prepared using a NanoAssemblr ${ }^{\mathrm{TM}}$ microfluidic mixer by the controlled mixing of ethanol solutions of lipid mixture with aqueous buffer.

For the preparation on LNPs from LDC, stock solutions of lipids were made up in ethanol with a lipid concentration of $10 \mathrm{mM}$. In an eppendorf tube, the lipid mixture of PEG-lipid (5.00 mol \%), DMPC (5.00 mol \%), and LDC ( $90.00 \mathrm{~mol} \%)$ was made up to a total volume of $0.25 \mathrm{~mL}$ with additional ethanol. The mixture was then heated to $43^{\circ} \mathrm{C}$ for 5 minutes in the oven. The volumetric compositions are given in Table S1 


\begin{tabular}{|l|l|l|l|l|l|}
\hline LDC & $\begin{array}{l}\text { LDC } \\
{[\text { Stock }]} \\
(\mathrm{mg} / \mathrm{mL})\end{array}$ & $\begin{array}{l}\text { Volume of } \\
\text { LDC Added } \\
(\mathrm{uL})\end{array}$ & $\begin{array}{l}\text { Volume of } \\
\text { DSPE-PEG } \\
\text { Added (uL) }\end{array}$ & $\begin{array}{l}\text { Volume of } \\
\text { DMPC Added } \\
(\mathrm{uL})\end{array}$ & $\begin{array}{l}\text { Volume of EtOH } \\
\text { Added (uL) }\end{array}$ \\
\hline MP-C14 & 10.0 & 204.0 & 36.9 & 8.9 & 0.0 \\
\hline MP-C16 & 10.4 & 199.0 & 35.0 & 8.5 & 7.9 \\
\hline MP-bC16 & 10.0 & 206.5 & 35.0 & 8.5 & 0.0 \\
\hline MP-C18 & 12.4 & 168.9 & 33.4 & 8.1 & 39.7 \\
\hline TG-C14 & 17.7 & 115.7 & 36.4 & 8.8 & 89.1 \\
\hline TG-C16 & 13.7 & 151.1 & 34.6 & 8.4 & 55.9 \\
\hline TG-bC16 & 16.6 & 124.8 & 34.6 & 8.4 & 82.3 \\
\hline
\end{tabular}

Table S1: LDC composition with stock [DSPE-PEG] $=10.0 \mathrm{mg} / \mathrm{mL}$ and stock [DMPC] $=10.0 \mathrm{mg} / \mathrm{mL}$ where the composition was 90: 5: 5 (LDC monomer: DMPC: DSPE-PEG). The drug loading was 15 wt\%.

For the evaluation of PEG-lipid in LNPs, the molar composition used in formulation was 1.5:10:38.5:50 (PEGLipid: Neutral Lipid: Cholesterol: Cationic/Ionizable Lipid) and with a lipid concentration of $12.5 \mathrm{mM}$. Stock solutions were made up in ethanol and the required amounts were transferred and then made to the required concentration by dilution with ethanol. The lipid mixture was made up in an Eppendorf tube from stock solutions of PEG-lipid (see Table A2.1), DSPC ( $20 \mathrm{mg} / \mathrm{mL}$; volume added: $27.2 \mathrm{uL}$ ), DOTMA (40 mg/mL; volume added: $57.6 \mathrm{uL}$ ), and cholesterol $(20 \mathrm{mg} / \mathrm{mL}$; volume added: $51.2 \mathrm{uL})$. The final volume was made up to a total volume of $0.55 \mathrm{~mL}$ with additional ethanol (see Table S2). The mixture was then sealed and heated to $43^{\circ} \mathrm{C}$ for 5 minutes in the oven. A stock solution of the dsDNA oligomer (5'-CGC GCG TAT ATA CGC GCG-3'; $50.00 \mathrm{mg} / \mathrm{mL}$ ) was made up in PBS buffer $\left(10.0 \mathrm{mM} \mathrm{Na}_{3} \mathrm{PO}_{4}, 0.154 \mathrm{M} \mathrm{NaCl}\right.$, and made to $\mathrm{pH} 7.4$ using concentrated $\mathrm{HCl}$, in Millipore water). To a glass vial, $6.0 \mathrm{uL}$ of the dsDNA stock solution was added and made up to a volume of 2.244 $\mathrm{mL}$ with sodium acetate buffer $(25.00 \mathrm{mM}$; $\mathrm{pH} 4$ using concentrated $\mathrm{HCl}$ to adjust the $\mathrm{pH}$ ).

\begin{tabular}{|l|l|l|l|}
\hline PEG-Lipid & $\begin{array}{l}\text { PEG-Lipids } \\
\text { Stock] }(\mathrm{mg} / \mathrm{mL})\end{array}$ & $\begin{array}{l}\text { Volume of PEG- } \\
\text { Lipid Added (uL) }\end{array}$ & $\begin{array}{l}\text { Volume of EtOH } \\
\text { Added (uL) }\end{array}$ \\
\hline PEG-G1-C14 & 19.2 & 13.6 & 400.4 \\
\hline PEG-G1-C16 & 18.6 & 14.4 & 399.6 \\
\hline PEG-G1-bC16 & 17.4 & 15.4 & 398.6 \\
\hline PEG-G1-C18 & 17.9 & 15.3 & 398.8 \\
\hline PEG-G2-C14 & 30.6 & 10.8 & 403.3 \\
\hline PEG-G2-C16 & 29.6 & 11.6 & 402.5 \\
\hline PEG-G2-bC16 & 29.0 & 11.8 & 402.3 \\
\hline PEG-G2-C18 & 30.3 & 11.7 & 402.4 \\
\hline DSG-PEG & 20.0 & 13.5 & 400.5 \\
\hline DSPE-PEG & 10.0 & 28.9 & 385.1 \\
\hline
\end{tabular}

Table S2: Concentrations of PEG-lipid solutions and required volumes for formulations containing 1.5 mol\% of different PEG-lipids. The composition for these SLNPs was 50: 10: 38.5: 1.5 (DOTMA: DSPC: Cholesterol: PEGlipid) with a DNA loading of $5.1 \mathrm{wt} \%$.

Prior to using, the NanoAssemblr ${ }^{\mathrm{TM}}$ cartridge was washed with PBS buffer in a $3 \mathrm{~mL}$ syringe (left port) and ethanol in a $3 \mathrm{~mL}$ syringe (right port) with a $12 \mathrm{~mL} / \mathrm{min}$. flow rate and a 1:1 (aqueous:ethanol ratio) flow ratio. A total of $4 \mathrm{~mL}$ of wash was collected and discarded. The NanoAssemblr ${ }^{\mathrm{TM}}$ microfluidic mixer was then used to make the LNPs. In a $3 \mathrm{~mL}$ syringe (left port) was loaded $2 \mathrm{~mL}$ PBS buffer and in another $3 \mathrm{~mL}$ syringe (right port) 
was loaded the $0.25 \mathrm{~mL}$ lipid mixture. The flow rate was set to $4 \mathrm{~mL} / \mathrm{min}$., the flow ratio to 3:1 (aqueous:EtOH), and the total volume collected $1 \mathrm{~mL}$ with the initial $300 \mathrm{uL}$ at the beginning and $50 \mathrm{uL}$ at the end being discarded.

For LNP containing LDCs, an aliquot $(0.50 \mathrm{~mL})$ of the collected formulation was then diluted to $0.40 \mathrm{mM}$ (thiopurine) with PBS buffer, transferred to a $3 \mathrm{~mL}$ Slide-A-Lyzer Dialysis Cassette G2 (10,000 molecular weight cutoff) and dialyzed against PBS buffer for 5 hours. The PBS was refreshed after 3 hours and the removal of ethanol from the formulation was monitored using potassium dichromate.

For PEG-lipid LNPs containing dsDNA, The collected formulation was immediately transferred to a $3 \mathrm{~mL}$ Slide-ALyzer ${ }^{\circledast}$ Dialysis Cassette $G 2$ (10,000 molecular weight cutoff) and dialyzed against PBS buffer for 6 hours. The PBS was refreshed after 3 hours and the removal of ethanol from the formulation was monitored using potassium dichromate (stable orange colour without greenish reaction indicated EtOH removal).

$Z_{\text {avg }}$ diameters of the LNPs were determined by dynamic light scattering (DLS) experiments (Brookhaven Instrument, ZetaPALS particle sizing software). The LNPs were then stored at $4{ }^{\circ} \mathrm{C}$.

\section{Particle stability assay methods}

The stability of the formulated LNPs was assessed under 6 conditions: LNPs were stored at 3 different temperatures; $4{ }^{\circ} \mathrm{C}, \mathrm{RT}$, and $37^{\circ} \mathrm{C}$, and incubated in 2 different media; PBS buffer and PBS buffer with $10 \%$ serum (by volume). To a polystyrene cuvette, $200 \mathrm{uL}$ of LNPs solution and $2.30 \mathrm{~mL}$ of PBS buffer were added and the diameters measured in a DLS experiment. Cuvettes were then sealed and incubated under the indicated conditions. Diameter measurements were taken regularly. To a polystyrene cuvette, $200 \mathrm{uL}$ of LNPs solution, $2.05 \mathrm{~mL}$ of PBS buffer, and $0.25 \mathrm{~mL}$ of serum were added and the diameters measured in a DLS experiment. Cuvettes were sealed and incubated under the indicated conditions.

Serum proteins scatter light and therefore interfere with direct determination of LNP diameter and PDI. The DLS values for LNPs together with serum represent a weighted size average between the LNPs and the serum particles. The weighted average is sample specific; changes of a given sample with time have relevance to the particle stability, but comparisons between samples is not possibile. 


\section{LDC particle stability}

\begin{tabular}{|l|l|l|l|}
\hline LDC & $\begin{array}{l}\text { LDC Diameter } \\
(\mathrm{nm})\end{array}$ & Polydispersity (PDI) & $\begin{array}{l}\text { Volume of PBS } \\
\text { buffer Added } \\
(\mathrm{mL})\end{array}$ \\
\hline MP-C14 & $227.5 \pm 11.3$ & $0.346 \pm 0.009$ & 2.59 \\
\hline MP-C16 & $216.7 \pm 7.7$ & $0.288 \pm 0.004$ & 2.44 \\
\hline MP-bC16 & $80.9 \pm 0.9$ & $0.277 \pm 0.003$ & 2.44 \\
\hline MP-C18 & $560.3 \pm 10.1$ & $0.358 \pm 0.010$ & 2.30 \\
\hline TG-C14 & $489.7 \pm 14.1$ & $0.343 \pm 0.007$ & 2.55 \\
\hline TG-C16 & $102.2 \pm 2.2$ & $0.237 \pm 0.006$ & 2.40 \\
\hline TG-bC16 & $64.8 \pm 1.2$ & $0.220 \pm 0.007$ & 2.40 \\
\hline
\end{tabular}

Table S3: Physical Characterizations of LDC formulations immediately following dialysis where the composition was 90: 5: 5 (LDC monomer: DMPC: DSPE-PEG). The drug loading was 15 wt\%.

\begin{tabular}{|l|l|l|l|}
\hline & $\begin{array}{l}\text { Day 1 } \\
\text { Size(nm); PDI }\end{array}$ & $\begin{array}{l}\text { Day 30 } \\
\text { Size(nm); PDI }\end{array}$ & $\begin{array}{l}\text { Day 60 } \\
\text { Size(nm); PDI }\end{array}$ \\
\hline MP-C14 & $227.5 \pm 11.3 ; 0.346 \pm$ & $438.4 \pm 5.4 ;$ & -- \\
& 0.009 & $0.267 \pm 0.004$ & \\
\hline MP-C16 & $216.7 \pm 7.7 ;$ & $220.2 \pm 2.3 ;$ & $205.4 \pm 2.1 ;$ \\
& $0.288 \pm 0.004$ & $0.209 \pm 0.013$ & $0.231 \pm 0.014$ \\
\hline MP-bC16 & $80.9 \pm 0.9 ;$ & $90.1 \pm 0.5 ;$ & $100.5 \pm 0.6 ;$ \\
& $0.277 \pm 0.003$ & $0.284 \pm 0.002$ & $0.296 \pm 0.002$ \\
\hline MP-C18 & $560.3 \pm 10.1 ; 0.358 \pm$ & $807.4 \pm 10.8 ; 0.371 \pm$ & -- \\
& 0.010 & 0.003 & -- \\
\hline TG-C14 & $489.7 \pm 14.1 ;$ & $1013.7 \pm 85.4 ;$ & \\
& $0.343 \pm 0.007$ & $0.427 \pm 0.036$ & $113.9 \pm 0.3 ;$ \\
\hline TG-C16 & $102.2 \pm 2.2 ;$ & $101.6 \pm 0.3 ;$ & $0.279 \pm 0.010$ \\
\hline TG-bC16 & $0.237 \pm 0.006$ & $0.215 \pm 0.008$ & $93.8 \pm 1.7 ;$ \\
& $64.8 \pm 1.2 ;$ & $73.8 \pm 1.3 ;$ & $0.314 \pm 0.012$ \\
\hline
\end{tabular}

Table S4: LDC storage stability at $4{ }^{\circ} \mathrm{C}$ in PBS buffer where the composition was 90: 5: 5 (LDC monomer: DMPC: DSPE-PEG). The drug loading was $15 \mathrm{wt} \%$. 


\begin{tabular}{|l|l|l|}
\hline LDC & $\begin{array}{l}\text { Day 1 } \\
\text { Size }(\mathrm{nm}) ; \text { PDI }\end{array}$ & $\begin{array}{l}\text { Day 30 } \\
\text { Size }(\mathrm{nm}) ; \text { PDI }\end{array}$ \\
\hline MP-C16 & $160.7 \pm 3.7 ;$ & $162.1 \pm 4.8 ;$ \\
& $0.399 \pm 0.004$ & $0.401 \pm 0.007$ \\
\hline MP-bC16 & $126.8 \pm 2.3 ;$ & $128.7 \pm 2.6 ;$ \\
& $0.406 \pm 0.002$ & $0.396 \pm 0.003$ \\
\hline TG-C16 & $134.8 \pm 3.1 ;$ & $138.4 \pm 1.7 ;$ \\
& $0.408 \pm 0.002$ & $0.405 \pm 0.004$ \\
\hline TG-bC16 & $128.6 \pm 7.4 ;$ & $131.7 \pm 2.5 ;$ \\
& $0.413 \pm 0.004$ & $0.405 \pm 0.003$ \\
\hline
\end{tabular}

Table S5: LDC storage stability at $4{ }^{\circ} \mathrm{C}$ in PBS buffer with 10\% serum where the composition was 90: 5: 5 (LDC monomer: DMPC: DSPE-PEG). The drug loading was $15 \mathrm{wt} \%$.

\begin{tabular}{|l|l|l|l|}
\hline LDC & $\begin{array}{l}\text { Day 1 } \\
\text { Size }(\mathrm{nm}) ; \text { PDI }\end{array}$ & $\begin{array}{l}\text { Day 30 } \\
\text { Size }(\mathrm{nm}) ; \text { PDI }\end{array}$ & $\begin{array}{l}\text { Day 60 } \\
\text { Size }(\mathrm{nm}) ; \text { PDI }\end{array}$ \\
\hline MP-C16 & $216.7 \pm 7.7 ;$ & $205.2 \pm 7.3 ;$ & $220.5 \pm 7.8 ;$ \\
& $0.288 \pm 0.004$ & $0.224 \pm 0.003$ & $0.212 \pm 0.003$ \\
\hline MP-bC16 & $80.9 \pm 0.9 ;$ & $83.3 \pm 0.9 ;$ & $81.3 \pm 0.9 ;$ \\
& $0.277 \pm 0.003$ & $0.259 \pm 0.003$ & $0.261 \pm 0.003$ \\
\hline TG-C16 & $102.2 \pm 2.2 ;$ & $121.6 \pm 2.6 ;$ & $124.4 \pm 2.7 ;$ \\
& $0.237 \pm 0.006$ & $0.147 \pm 0.004$ & $0.164 \pm 0.004$ \\
\hline TG-bC16 & $64.8 \pm 1.2 ;$ & $73.8 \pm 1.4 ;$ & $69.5 \pm 1.3 ;$ \\
& $0.220 \pm 0.007$ & $0.275 \pm 0.009$ & $0.219 \pm 0.007$ \\
\hline
\end{tabular}

Table S6: LDC storage stability at RT in PBS buffer where the composition was 90: 5: 5 (LDC monomer: DMPC: DSPE-PEG). The drug loading was $15 \mathrm{wt} \%$.

\begin{tabular}{|l|l|l|l|}
\hline LDC & $\begin{array}{l}\text { Day 1 } \\
\text { Size }(\mathrm{nm}) ; \text { PDI }\end{array}$ & $\begin{array}{l}\text { Day 30 } \\
\text { Size }(\mathrm{nm}) ; \text { PDI }\end{array}$ & $\begin{array}{l}\text { Day 60 } \\
\text { Size }(\mathrm{nm}) ; \text { PDI }\end{array}$ \\
\hline MP-C16 & $160.7 \pm 3.7 ;$ & $230.8 \pm 5.3 ;$ & $309.0 \pm 7.1 ;$ \\
& $0.399 \pm 0.004$ & $0.413 \pm 0.004$ & $0.418 \pm 0.004$ \\
\hline MP-bC16 & $126.8 \pm 2.3 ;$ & $163.5 \pm 3.0 ;$ & $123.3 \pm 2.3 ;$ \\
& $0.406 \pm 0.002$ & $0.397 \pm 0.002$ & $0.353 \pm 0.002$ \\
\hline TG-C16 & $134.8 \pm 3.1 ;$ & $134.4 \pm 3.1 ;$ & $139.6 \pm 3.2 ;$ \\
& $0.408 \pm 0.002$ & $0.407 \pm 0.002$ & $0.413 \pm 0.002$ \\
\hline TG-bC16 & $128.6 \pm 7.4 ;$ & $126.8 \pm 7.3 ;$ & $199.8 \pm 11.5 ; 0.433 \pm$ \\
& $0.413 \pm 0.004$ & $0.401 \pm 0.004$ & 0.004 \\
\hline
\end{tabular}

Table S7: LDC storage stability at RT in PBS buffer with 10\% serum where the composition was 90: $5: 5$ (LDC monomer: DMPC: DSPE-PEG). The drug loading was 15 wt\%. 


\begin{tabular}{|l|l|l|l|}
\hline LDC & $\begin{array}{l}\text { Day 1 } \\
\text { Size(nm); PDI }\end{array}$ & $\begin{array}{l}\text { Day 2 } \\
\text { Size }(\mathrm{nm}) ; \text { PDI }\end{array}$ & $\begin{array}{l}\text { Day 3 } \\
\text { Size }(\mathrm{nm}) ; \text { PDI }\end{array}$ \\
\hline MP-C16 & $216.7 \pm 7.7 ;$ & $262.1 \pm 5.0 ;$ & $260.5 \pm 2.5 ; 0.221 \pm$ \\
& $0.288 \pm 0.004$ & $0.208 \pm 0.007$ & 0.010 \\
\hline MP-bC16 & $80.9 \pm 0.9 ;$ & $101.5 \pm 4.2 ;$ & $95.0 \pm 4.1 ;$ \\
& $0.277 \pm 0.003$ & $0.315 \pm 0.007$ & $0.309 \pm 0.008$ \\
\hline TG-C16 & $102.2 \pm 2.2 ;$ & $189.3 \pm 5.7 ;$ & $182.5 \pm 0.7 ;$ \\
& $0.237 \pm 0.006$ & $0.208 \pm 0.011$ & $0.173 \pm 0.005$ \\
\hline TG-bC16 & $64.8 \pm 1.2 ;$ & $72.9 \pm 1.9 ;$ & $76.3 \pm 1.8 ;$ \\
& $0.220 \pm 0.007$ & $0.251 \pm 0.003$ & $0.258 \pm 0.013$ \\
\hline
\end{tabular}

Table S8: LDC storage stability at $37^{\circ} \mathrm{C}$ in PBS buffer where the composition was 90: 5: 5 (LDC monomer: DMPC: DSPE-PEG). The drug loading was 15 wt\%.

\begin{tabular}{|l|l|l|l|}
\hline LDC & Day 1 & $\begin{array}{l}\text { Day 2 } \\
\text { Size }(\mathrm{nm}) ; \text { PDI }\end{array}$ & $\begin{array}{l}\text { Day 3 } \\
\text { Size }(\mathrm{nm}) ; \text { PDI }\end{array}$ \\
\hline MP-C16 & $160.7 \pm 3.7 ;$ & $189.3 \pm 2.3 ;$ & $186.0 \pm 1.3 ;$ \\
& $0.399 \pm 0.004$ & $0.393 \pm 0.003$ & $0.402 \pm 0.008$ \\
\hline MP-bC16 & $126.8 \pm 2.3 ;$ & $139.6 \pm 16.8 ; 0.379 \pm$ & $134.4 \pm 2.6 ;$ \\
& $0.406 \pm 0.002$ & 0.001 & $0.388 \pm 0.005$ \\
\hline TG-C16 & $134.8 \pm 3.1 ;$ & $143.3 \pm 7.1 ;$ & $139.2 \pm 3.0 ;$ \\
& $0.408 \pm 0.002$ & $0.378 \pm 0.008$ & $0.388 \pm 0.004$ \\
\hline TG-bC16 & $128.6 \pm 7.4 ;$ & $154.3 \pm 15.1 ; 0.387 \pm$ & $155.4 \pm 2.8 ;$ \\
& $0.413 \pm 0.004$ & 0.001 & $0.375 \pm 0.004$ \\
\hline
\end{tabular}

Table S9: LDC storage stability at $37^{\circ} \mathrm{C}$ in PBS buffer with 10\% serum where the composition was 90: 5: 5 (LDC monomer: DMPC: DSPE-PEG). The drug loading was 15 wt\%.

\section{PEG-lipid particle stability}

\begin{tabular}{|l|l|l|}
\hline PEG-Lipids & SNP Diameter $(\mathrm{nm})$ & Polydispersity \\
\hline PEG-G1-C14 & $104.5 \pm 1.5$ & $0.307 \pm 0.006$ \\
\hline PEG-G1-C16 & $76.9 \pm 0.7$ & $0.259 \pm 0.004$ \\
\hline PEG-G1-bC16 & $156.1 \pm 1.9$ & $0.248 \pm 0.008$ \\
\hline PEG-G1-C18 & $146.1 \pm 1.4$ & $0.335 \pm 0.003$ \\
\hline PEG-G2-C14 & $181.4 \pm 21.4$ & $0.280 \pm 0.137$ \\
\hline PEG-G2-C16 & $211.1 \pm 4.8$ & $0.373 \pm 0.016$ \\
\hline PEG-G2-bC16 & $119.5 \pm 7.1$ & $0.351 \pm 0.025$ \\
\hline PEG-G2-C18 & $165.6 \pm 11.3$ & $0.394 \pm 0.031$ \\
\hline DSG-PEG & $80.5 \pm 0.9$ & $0.268 \pm 0.005$ \\
\hline DSPE-PEG & $89.0 \pm 0.4$ & $0.297 \pm 0.002$ \\
\hline
\end{tabular}

Table S10: Physical characterization of the LNP formulations immediately following preparation for formulations containing 1.5 mol\% of different PEG-lipids. The composition for these SLNPs was 50: 10: 38.5: 1.5 (DOTMA: DSPC: Cholesterol: PEG-lipid) with a DNA loading of $5.1 \mathrm{wt} \%$. 


\begin{tabular}{|l|l|l|}
\hline Formulation & $\begin{array}{l}\text { Day } 1 \\
\text { Size }(\mathrm{nm}) ; \text { PDI }\end{array}$ & $\begin{array}{l}\text { Day } 8 \\
\text { Size }(\mathrm{nm}) ; \text { PDI }\end{array}$ \\
\hline PEG-G1-C14 & $104.5 \pm 1.5 ; 0.307 \pm 0.006$ & $166.7 \pm 2.4 ; 0.222 \pm 0.004$ \\
\hline PEG-G1-C16 & $76.9 \pm 0.7 ; 0.259 \pm 0.004$ & $144.2 \pm 1.3 ; 0.212 \pm 0.003$ \\
\hline PEG-G1-bC16 & $156.1 \pm 1.9 ; 0.248 \pm 0.008$ & $176.6 \pm 2.1 ; 0.232 \pm 0.007$ \\
\hline PEG-G1-C18 & $146.1 \pm 1.4 ; 0.335 \pm 0.003$ & $172.0 \pm 1.6 ; 0.280 \pm 0.002$ \\
\hline DSG-PEG & $80.5 \pm 0.9 ; 0.268 \pm 0.005$ & $118.2 \pm 1.3 ; 0.268 \pm 0.005$ \\
\hline DSPE-PEG & $89.0 \pm 0.4 ; 0.297 \pm 0.002$ & $126.2 \pm 0.6 ; 0.293 \pm 0.002$ \\
\hline
\end{tabular}

Table S11: LNP storage stability at RT in PBS buffer for formulations containing 1.5 mol\% of different PEG-lipids. The composition for these SLNPs was 50: 10: 38.5: 1.5 (DOTMA: DSPC: Cholesterol: PEG-lipid) with a DNA loading of $5.1 \mathrm{wt} \%$.

\begin{tabular}{|l|l|l|}
\hline Formulation & $\begin{array}{l}\text { Day } 1 \\
\text { Size }(\mathrm{nm}) ; \text { PDI }\end{array}$ & $\begin{array}{l}\text { Day } 8 \\
\text { Size }(\mathrm{nm}) ; \text { PDI }\end{array}$ \\
\hline PEG-G1-C14 & $134.1 \pm 1.1 ; 0.350 \pm 0.001$ & $197.1 \pm 1.6 ; 0.345 \pm 0.001$ \\
\hline PEG-G1-C16 & $129.6 \pm 1.4 ; 0.340 \pm 0.000$ & $225.1 \pm 2.4 ; 0.381 \pm 0.005$ \\
\hline PEG-G1-bC16 & $178.3 \pm 5.5 ; 0.337 \pm 0.005$ & $249.1 \pm 7.7 ; 0.338 \pm 0.005$ \\
\hline PEG-G1-C18 & $166.8 \pm 4.4 ; 0.345 \pm 0.003$ & $290.7 \pm 7.7 ; 0.376 \pm 0.003$ \\
\hline DSG-PEG & $117.9 \pm 2.2 ; 0.357 \pm 0.004$ & $223.7 \pm 4.2 ; 0.383 \pm 0.004$ \\
\hline DSPE-PEG & $129.0 \pm 4.8 ; 0.349 \pm 0.005$ & $212.3 \pm 7.9 ; 0.389 \pm 0.006$ \\
\hline
\end{tabular}

Table S12: SLNP storage stability at RT in PBS buffer with $10 \%$ serum for formulations containing 1.5 mol\% of different PEG-lipids. The composition for these SLNPs was 50: 10: 38.5: 1.5 (DOTMA: DSPC: Cholesterol: PEGlipid) with a DNA loading of $5.1 \mathrm{wt} \%$.

\begin{tabular}{|l|l|l|l|}
\hline Formulation & $\begin{array}{l}\text { Day } 1 \\
\text { Size }(\mathrm{nm}) ; \text { PDI }\end{array}$ & $\begin{array}{l}\text { Day } 2 \\
\text { Size }(\mathrm{nm}) ; \text { PDI }\end{array}$ & $\begin{array}{l}\text { Day } 5 \\
\text { Size }(\mathrm{nm}) ; \text { PDI }\end{array}$ \\
\hline PEG-G1-C14 & $104.5 \pm 1.5 ; 0.307 \pm 0.006$ & $175.2 \pm 1.1 ; 0.194 \pm 0.010$ & $228.6 \pm 1.4 ; 0.164 \pm 0.008$ \\
\hline PEG-G1-C16 & $76.9 \pm 0.7 ; 0.259 \pm 0.004$ & $157.2 \pm 1.4 ; 0.204 \pm 0.002$ & $194.3 \pm 1.7 ; 0.131 \pm 0.001$ \\
\hline PEG-G1-bC16 & $156.1 \pm 1.9 ; 0.248 \pm 0.008$ & $198.7 \pm 7.5 ; 0.088 \pm 0.042$ & $180.5 \pm 6.8 ; 0.142 \pm 0.012$ \\
\hline PEG-G1-C18 & $146.1 \pm 1.4 ; 0.335 \pm 0.003$ & $173.0 \pm 1.4 ; 0.245 \pm 0.008$ & $216.3 \pm 1.8 ; 0.204 \pm 0.007$ \\
\hline DSG-PEG & $80.5 \pm 0.9 ; 0.268 \pm 0.005$ & $299.1 \pm 16.0 ; 0.230 \pm 0.004$ & $192.1 \pm 10.3 ; 0.187 \pm 0.003$ \\
\hline DSPE-PEG & $89.0 \pm 0.4 ; 0.297 \pm 0.002$ & $136.5 \pm 1.7 ; 0.255 \pm 0.005$ & $175.4 \pm 2.2 ; 0.146 \pm 0.003$ \\
\hline
\end{tabular}

Table S13: LNP stability at $37^{\circ} \mathrm{C}$ in PBS buffer for formulations containing $1.5 \mathrm{~mol} \%$ of different PEG-lipids. The composition for these SLNPs was 50: 10: 38.5: 1.5 (DOTMA: DSPC: Cholesterol: PEG-lipid) with a DNA loading of $5.1 \mathrm{wt} \%$. 


\begin{tabular}{|l|l|l|l|}
\hline Formulation & $\begin{array}{l}\text { Day } 1 \\
\text { Size }(\mathrm{nm}) ; \mathrm{PDI}\end{array}$ & $\begin{array}{l}\text { Day } 2 \\
\text { Size }(\mathrm{nm}) ; \text { PDI }\end{array}$ & $\begin{array}{l}\text { Day } 5 \\
\text { Size }(\mathrm{nm}) ; \text { PDI }\end{array}$ \\
\hline PEG-G1-C14 & $134.1 \pm 1.1 ; 0.350 \pm 0.001$ & $190.1 \pm 2.8 ; 0.291 \pm 0.012$ & $194.8 \pm 2.9 ; 0.349 \pm 0.014$ \\
\hline PEG-G1-C16 & $129.6 \pm 1.4 ; 0.340 \pm 0.000$ & $171.5 \pm 1.9 ; 0.344 \pm 0.005$ & $165.3 \pm 1.8 ; 0.351 \pm 0.005$ \\
\hline PEG-G1-bC16 & $178.3 \pm 5.5 ; 0.337 \pm 0.005$ & $211.1 \pm 16.8 ; 0.194 \pm 0.069$ & $220.0 \pm 14.4 ; 0.363 \pm 0.047$ \\
\hline PEG-G1-C18 & $166.8 \pm 4.4 ; 0.345 \pm 0.003$ & $237.9 \pm 23.3 ; 0.320 \pm 0.042$ & $204.2 \pm 19.9 ; 0.363 \pm 0.048$ \\
\hline DSG-PEG & $117.9 \pm 2.2 ; 0.357 \pm 0.004$ & $167.9 \pm 9.4 ; 0.366 \pm 0.002$ & $120.5 \pm 6.7 ; 0.356 \pm 0.002$ \\
\hline DSPE-PEG & $129.0 \pm 4.8 ; 0.349 \pm 0.005$ & $149.4 \pm 6.0 ; 0.370 \pm 0.005$ & $197.8 \pm 7.9 ; 0.365 \pm 0.005$ \\
\hline
\end{tabular}

Table S14: LNP stability at $37^{\circ} \mathrm{C}$ in PBS buffer with $10 \%$ serum for formulations containing $1.5 \mathrm{~mol} \%$ of different PEG-lipids. The composition for these SLNPs was 50: 10: 38.5: 1.5 (DOTMA: DSPC: Cholesterol: PEG-lipid) with a DNA loading of $5.1 \mathrm{wt} \%$.

\begin{tabular}{|l|l|l|}
\hline PEG-Lipid & $\begin{array}{l}\text { Particle Diameter } \\
(\mathrm{nm})\end{array}$ & PDI \\
\hline DMG-PEG & 65.2 & 0.249 \\
\hline PEG-G2-bC16 & 73.6 & 0.112 \\
\hline PEG-G2-C16 & 86.1 & 0.262 \\
\hline
\end{tabular}

Table S15: The physical characterizations of formulations using the proprietary lipid mix are shown above. The DNA loading was 5.1\% with a corresponding charge ratio of 5 . The composition of the commercial mix was 50 : 10: 38: 1.5 (Ionisable lipid: DSPC: Cholesterol: PEG-lipid) where the final 0.5 mol\% was a fluorescent lipidmarker.

\begin{tabular}{|l|l|l|}
\hline PEG-Lipid & $\begin{array}{l}\text { Day } 1 \\
\text { Size }(\mathrm{nm}) ; \text { PDI }\end{array}$ & $\begin{array}{l}\text { Day 8 } \\
\text { Size }(\mathrm{nm}) ; \\
\text { PDI }\end{array}$ \\
\hline DMG-PEG & $65.1 ; 0.248$ & $304.0 ; 0.263$ \\
\hline PEG-G2-bC16 & $74.2 ; 0.122$ & $124.3 ; 0.183$ \\
\hline PEG-G2-C16 & $86.1 ; 0.262$ & $259.1 ; 0.183$ \\
\hline
\end{tabular}

Table S16: LNP stability at $37^{\circ} \mathrm{C}$ in PBS buffer for formulations using the proprietary lipid mix. The DNA loading was $5.1 \%$ with a corresponding charge ratio of 5 . The composition of the commercial mix was 50: 10: $38: 1.5$ (Ionisable lipid: DSPC: Cholesterol: PEG-lipid) where the final 0.5 mol\% was a fluorescent lipid-marker. 


\section{LNP imaging}

Cryo-SEM images were prepared by applying the sample, doped with $5 \mathrm{~nm}$ gold particles for calibration, to a lacey carbon EM grid. Filter paper was then used to blot the sample and to dehydrate it. The grid was then frozen in liquid ethane. For the LNP containing LDCs, every sample preparation protocol resulted in dense particle aggregates (Figure S11). Individual particles are evident in these masses, with approximately the sizes expected from the DLS experiments; hydrophobic association apparently drives the aggregation during sample preparation.

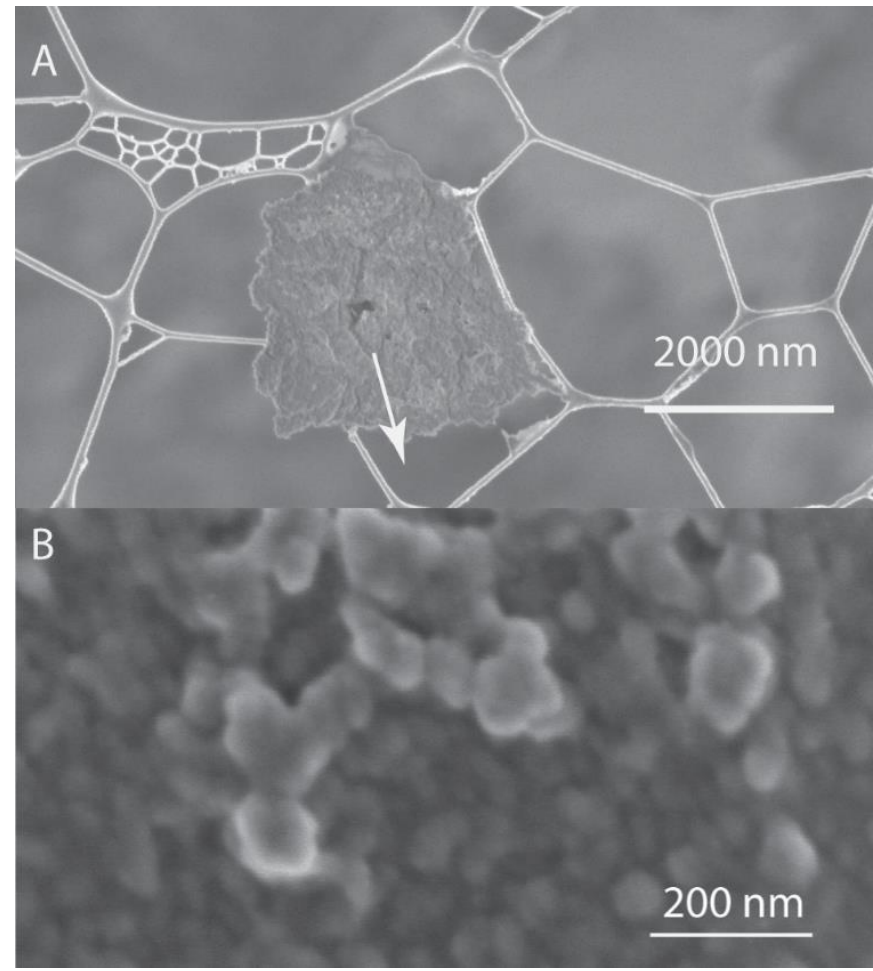

Figure S11: SEM images of LDC formulated from TGC16. An aliquot of the LDC mixture $(440 \mu \mathrm{M})$ in PBS (0.1 M NaCl; $10 \mathrm{mM}$ phosphate $\mathrm{pH} 7.4$ ) was dried on a lacey carbon grid prior to imaging. Panel $A$ shows one of the large aggregates held on the grid; panel $B$ shows an expanded section of this aggregate. 
Preparation steps also damaged the samples from PEG-lipid compositions resulting in aggregation and coalescence. Some images (Figure S12 lower) show regions that are irregular on the particle surface suggesting that particle dehydration during the preparation has fractured the particle.

Figure S12: Cryo-SEM image of PEG-G 1 -C16 sample showing particles with a generally spherical morphology.

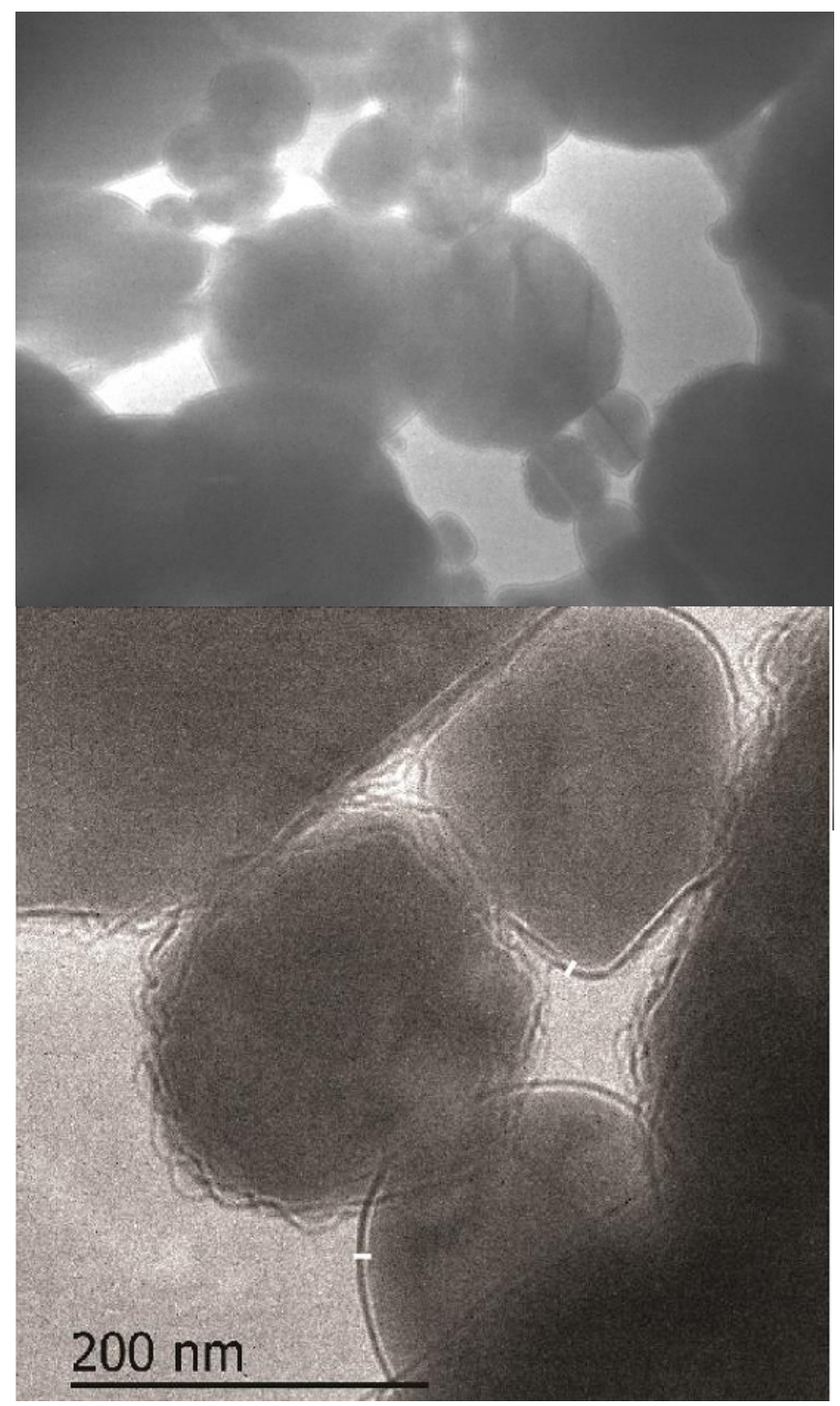




\section{Bioassay methods}

MCF7 cells were seeded on 24-well plates at $5.0 \times 10^{4}$ cells/well, $18 \mathrm{~h}$ later were treated with the indicated compounds in growth medium (Dulbecco's Modified Eagle's Medium [DMEM] supplemented with 10\% Bovine Growth Serum [BGS]). DMSO was used at a concentration of $400 \mu \mathrm{M}$ in growth medium as a control for 6-TG and 6-MP, and $\mathrm{Na}_{3} \mathrm{PO}_{4}$ buffer $\left(\mathrm{pH} 7,\left[\mathrm{Na}_{3} \mathrm{PO}_{4}\right]=10 \mathrm{mM}[\mathrm{NaCl}]=0.1 \mathrm{M}\right)$ was used at a concentration of $40 \mu \mathrm{M}$ in growth medium as a control for 6TG-C16, 6TG-bC16, 6MP-C16, and 6MP-bC16. After $72 \mathrm{~h}$ of treatment, cells were washed with phosphate-buffered saline (PBS), fixed for 10 mins with $4 \%$ paraformaldehyde in PBS, stained for 30 mins with $0.1 \%$ crystal violet in $\mathrm{H}_{2} \mathrm{O}$, washed twice with $\mathrm{H}_{2} \mathrm{O}$, and let dry. $10 \%$ acetic acid was added to wells and incubated on a shaker for 10 mins. Absorbance was measured at $590 \mathrm{~nm}$ using a PerkinElmer Victor ${ }^{3} \mathrm{~V}$ 1420 multilabel plate counter. The absorbance from stained wells without cells was subtracted from experimental values to eliminate background absorbance from crystal violet adhering to the plate. \% viability was calculated as (Absorbance compounds/Absorbance control buffer)*100.

A one-way ANOVA was performed for the experiments on MCF-7 cells: TG, $F(7,16)=243.8$ and $P<0.001 ;$ MP, $F$ $(7,16)=18.77$ and $P<0.001 ;$ TG-C16, $F(5,12)=23.58$ and $P<0.001 ;$ TG-bC16, $F(5,12)=24.97$ and $P<0.001$; MPC16, $F(5,12)=18.21$ and $P<0.001$; MP-C16, $F(5,12)=18.16$ and $P<0.001$, followed by Tukey's multiplecomparison post hoc test to determine whether there were significant differences between groups. ${ }^{*}, P<0.05$; $* *, P<0.01 ; * * *, P<0.001$. Error bars represent standard deviation.

A one-way ANOVA was also performed for the experiments on HeLa cells: TG, $F(7,16)=24.69, P<0.001 ; \mathrm{MP}, F$ $(7,16)=13.37$ and $P<0.001$; TG-C16, $F(6,14)=26.51$ and $P<0.001$; TG-bC16, $F(6,14)=2.06$ and $P>0.05$; MPC16, $F(6,17)=2.24$ and $P>0.05 ;$ MP-bC16, $F(6,14)=0.57$ and $P>0.05$, followed by Tukey's multiple-comparison post hoc test to determine whether there were significant differences between groups. ${ }^{* *}, P<0.01 ; * * *$, $P<0.001$. Error bars represent standard deviation.

\section{Cell viability data}

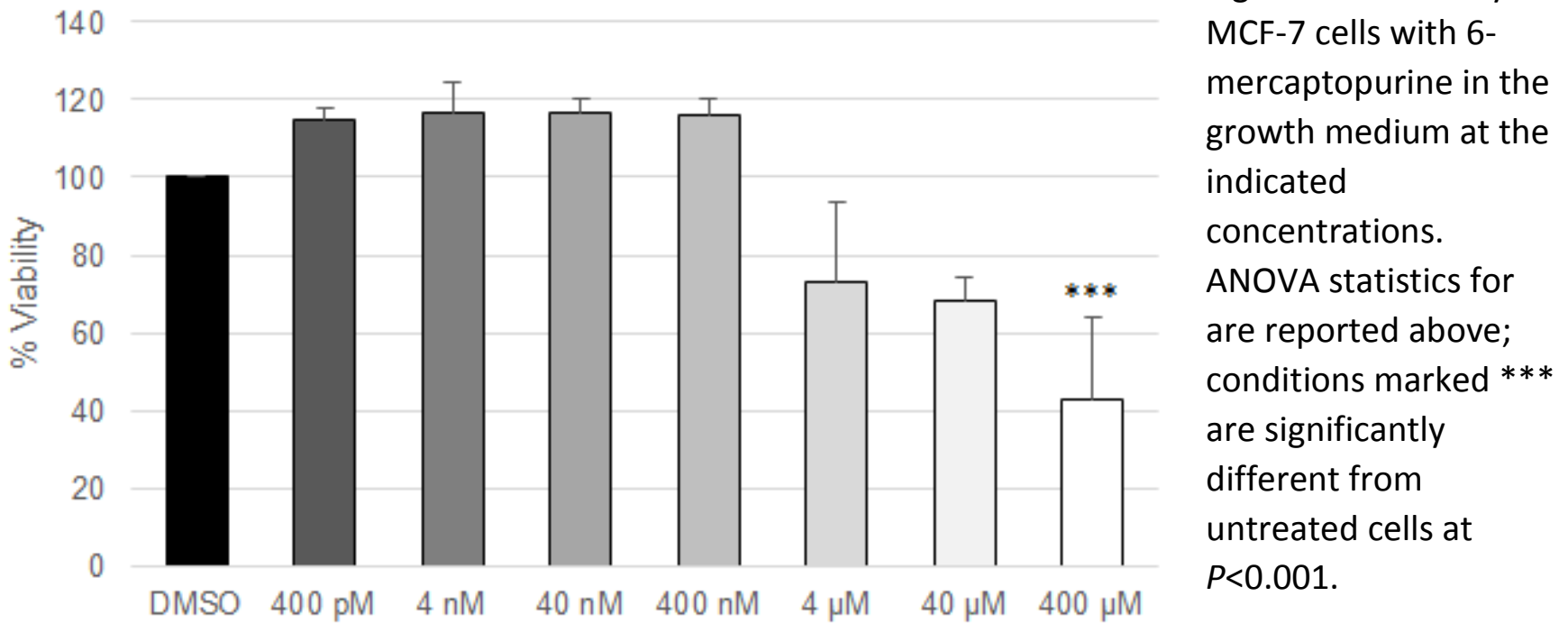




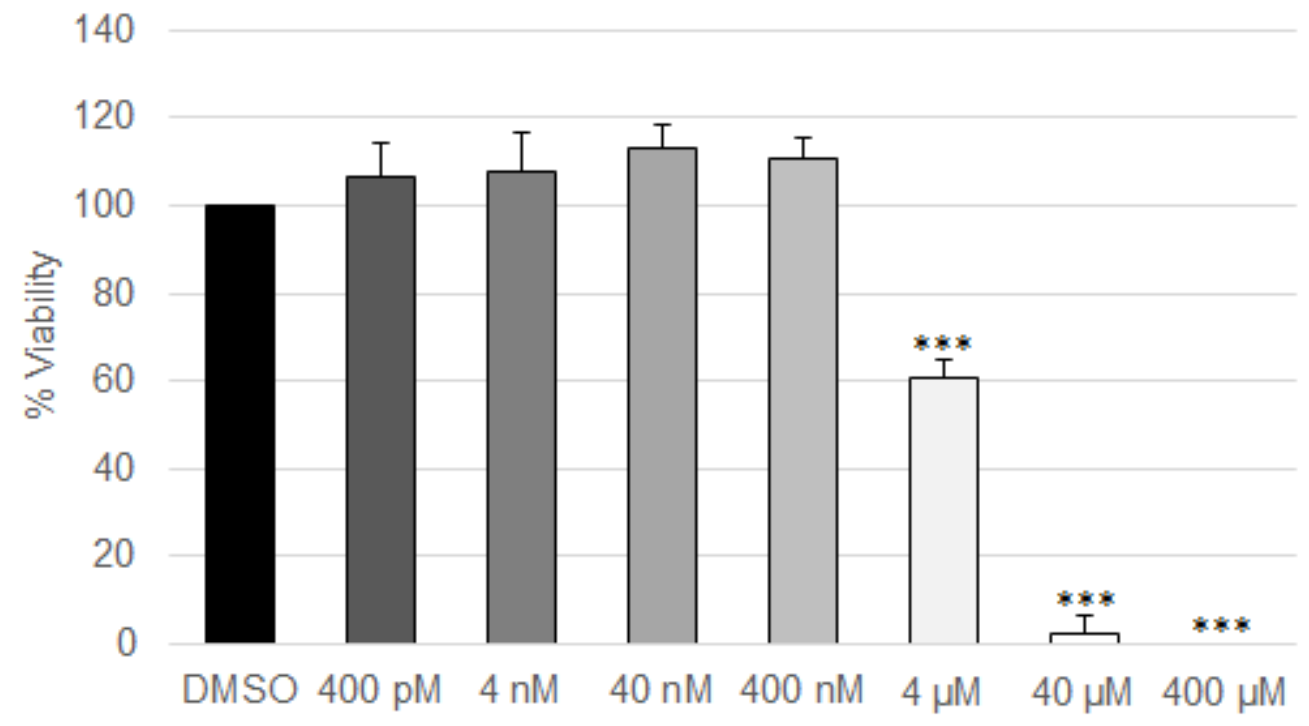

Figure S14: Viability of MCF-7 cells with 6-thioguanine in the growth medium at the indicated concentrations. ANOVA statistics for this experiment are reported above; conditions marked $* * *$ are significantly different from untreated cells at $P<0.001$.

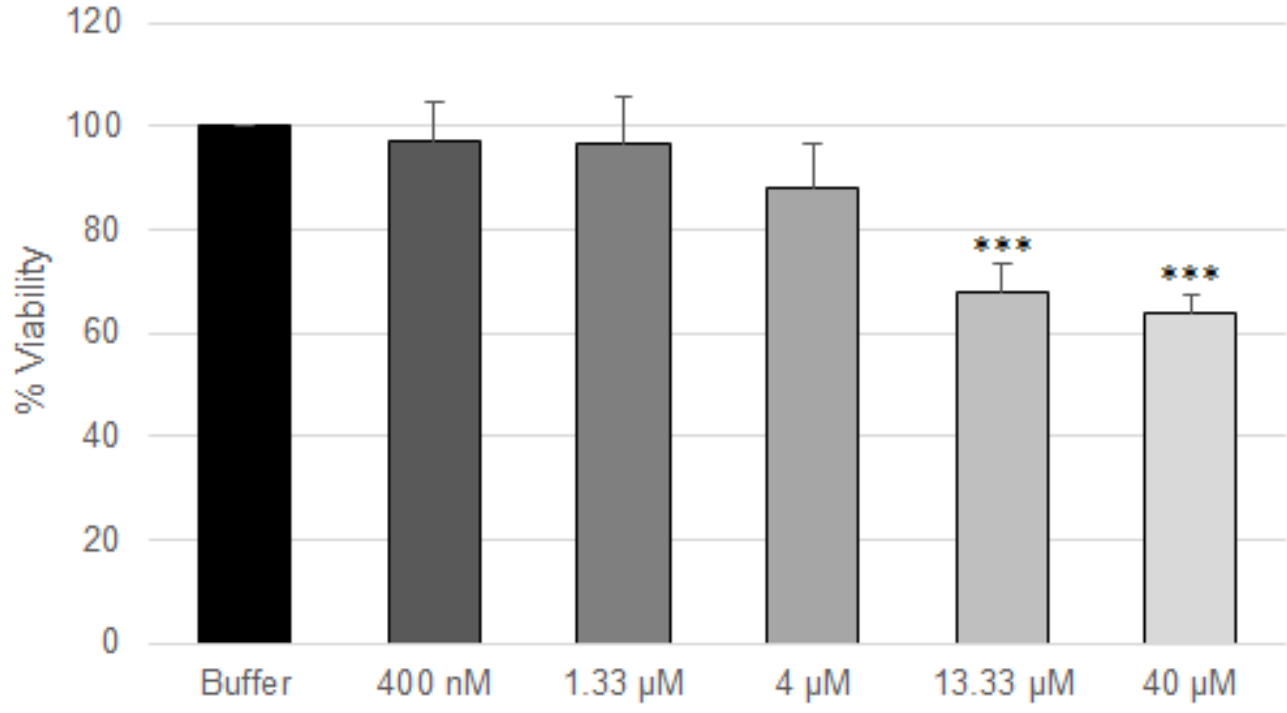

Figure S15: Viability of MCF-7 cells with MP-C16 in the growth medium at the indicated concentrations. Statistics for this experiment are reported above; conditions marked $* * *$ are significantly different from untreated cells at $P<0.001$. 


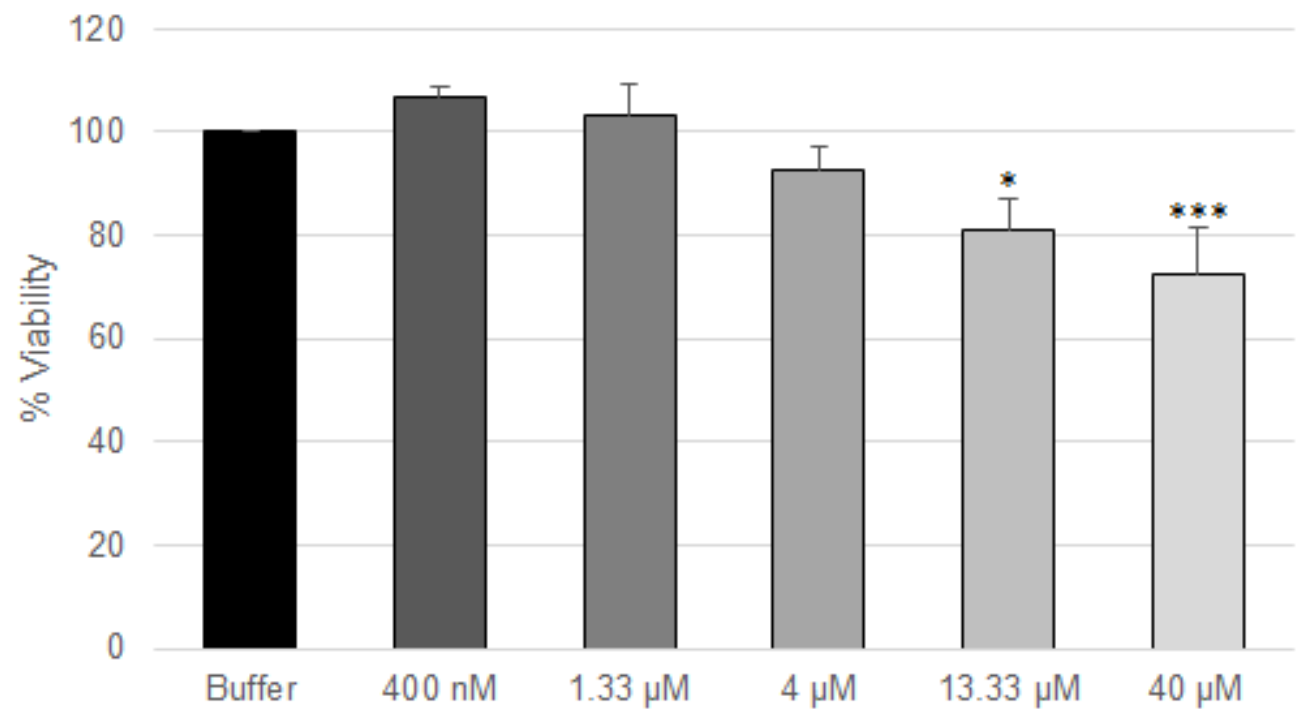

Figure S16: Viability of MCF-7 cells with MP-bC16 in the growth medium at the indicated concentrations. ANOVA statistics for this experiment are reported above; conditions marked * and *** are significantly different from untreated cells at $\mathrm{P}<0.05$ and $P<0.001$ respectively.

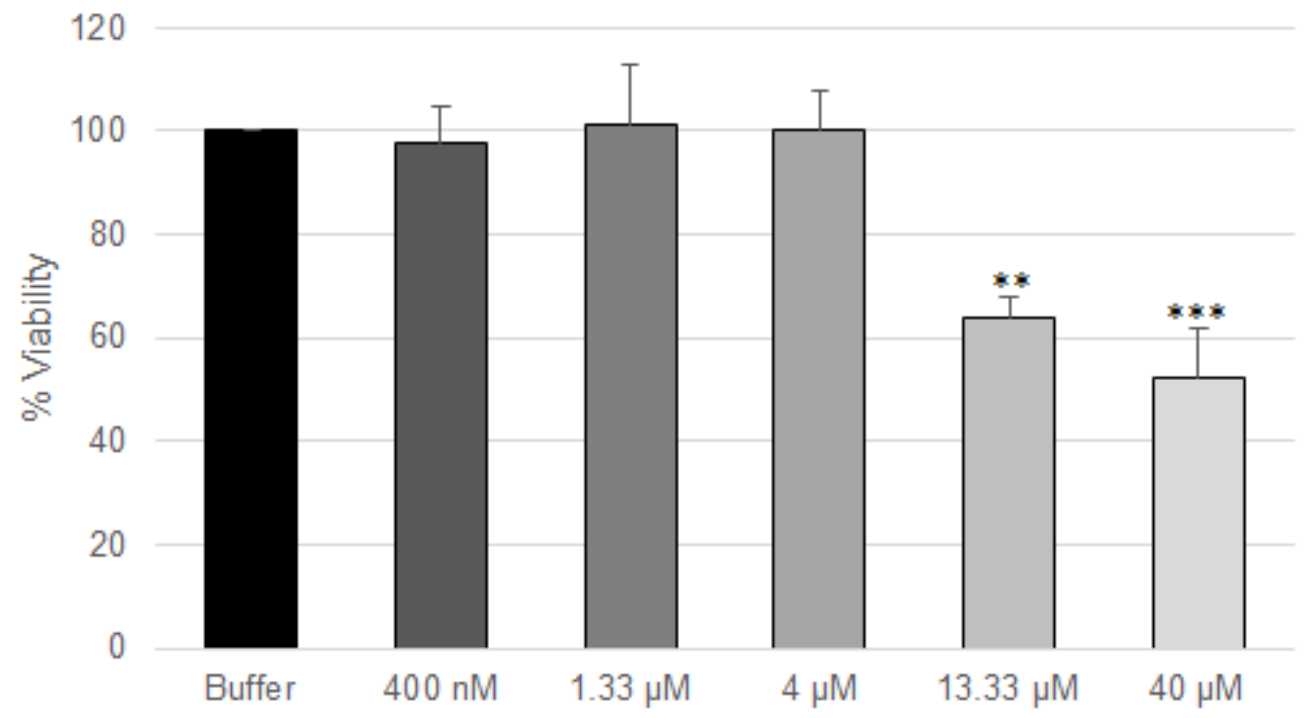

Figure S17: Viability of MCF-7 cells with TG-C16 in the growth medium at the indicated concentrations. ANOVA statistics for this experiment are reported above; conditions marked ${ }^{* *}$ and $* * *$ are significantly different from untreated cells at $P<0.01$ and $P<0.001$ respectively. 


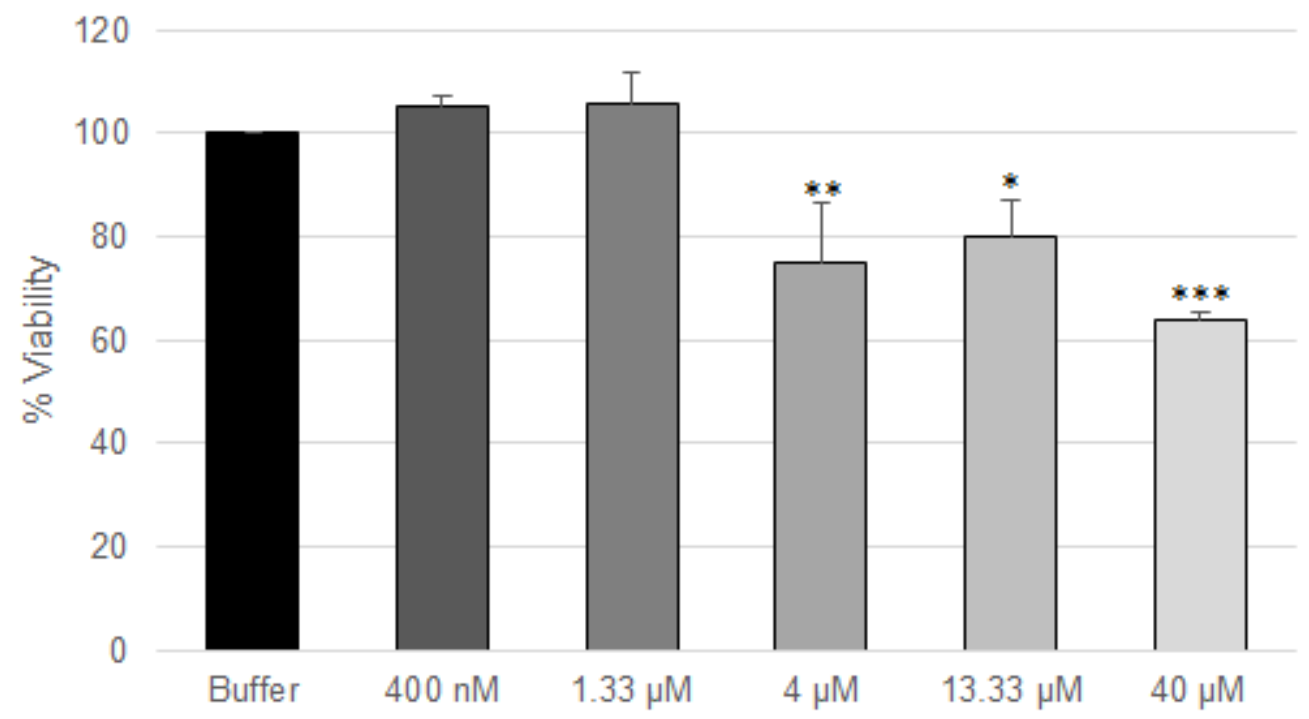

Figure S18: Viability of MCF-7 cells with TG-bC16 in the growth medium at the indicated concentrations. ANOVA statistics for this experiment are reported above; conditions marked $*, * *$ and $* * *$ are significantly different from untreated cells at $\mathrm{P}<0.05, \mathrm{P}<0.01$ and $P<0.001$ respectively.

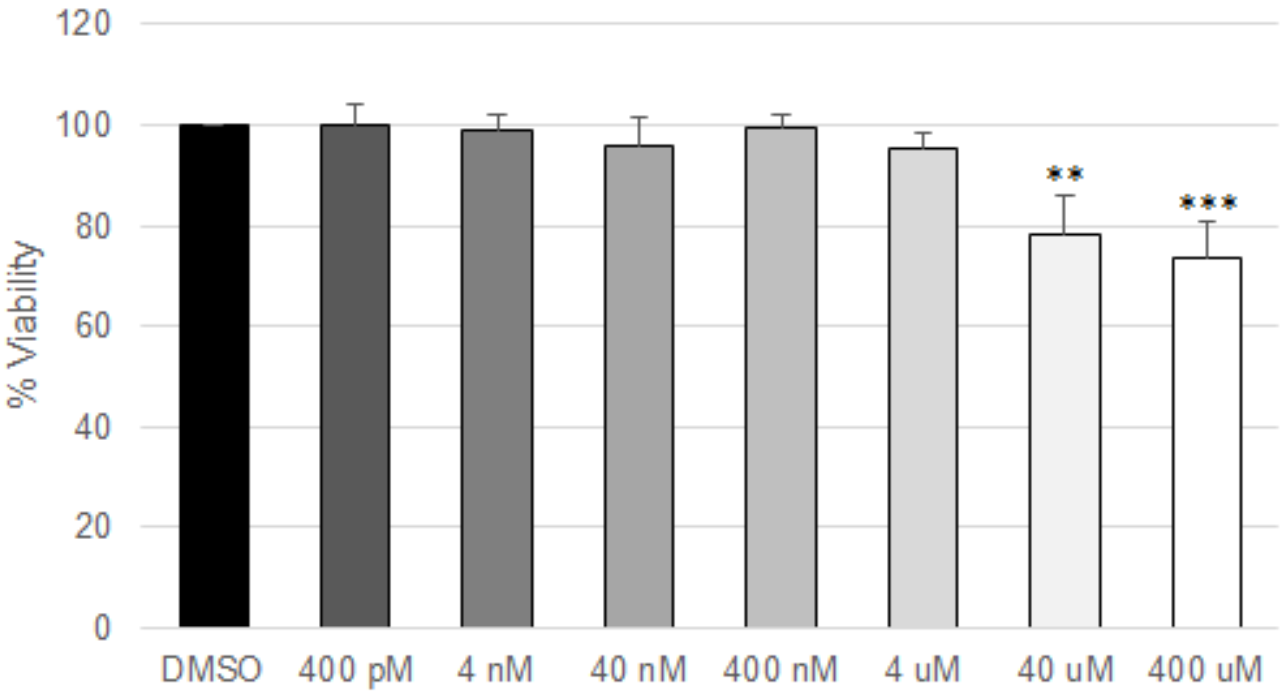

Figure S19: Viability of HeLa cells with 6-mercaptopurine in the growth medium at the indicated concentrations. ANOVA statistics for this experiment are reported above; conditions marked ${ }^{* *}$ and $* * *$ are significantly different from untreated cells at $P<0.01$ and $P<0.001$ respectively. 


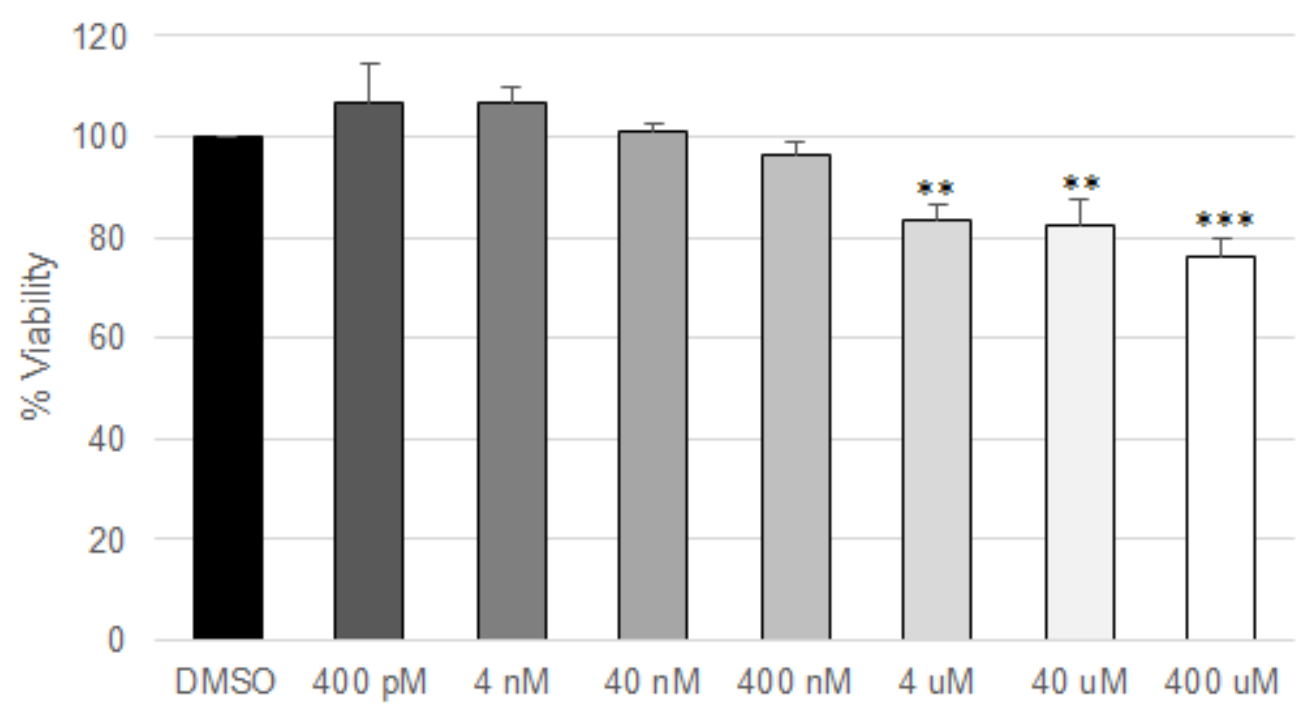

Figure S20: Viability of HeLa cells with 6-thioguanine in the growth medium at the indicated concentrations. ANOVA statistics for this experiment are reported above; conditions marked ${ }^{* *}$ and *** are significantly different from untreated cells at $P<0.01$ and $P<0.001$ respectively.

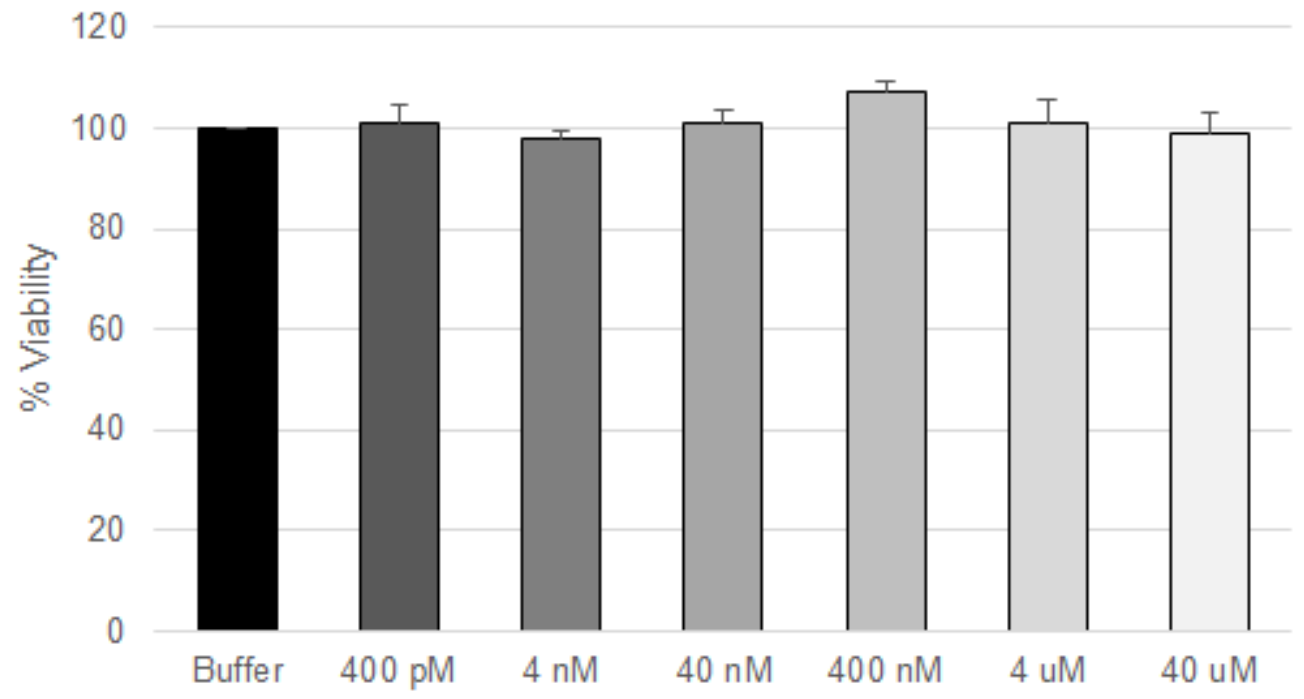

Figure S21: Viability of HeLa cells with MP-C16 in the growth medium at the indicated concentrations. ANOVA statistics for this experiment are reported above. 


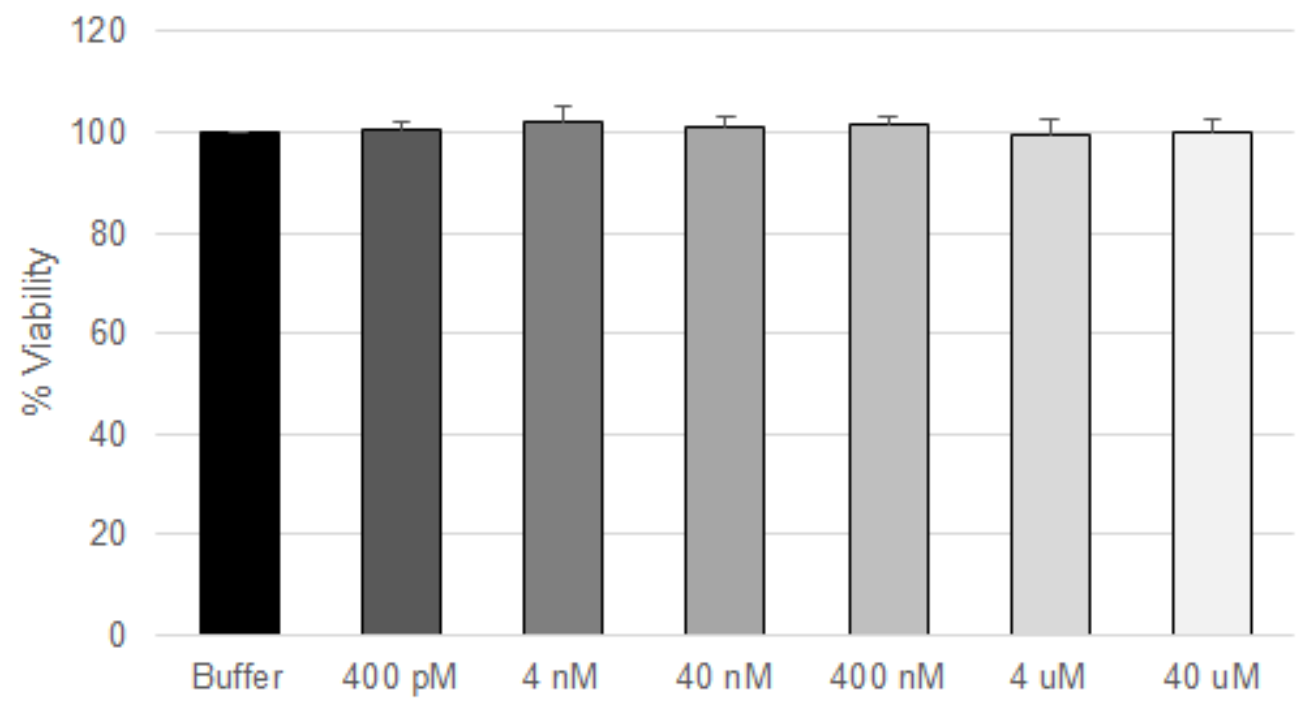

Figure S22: Viability of HeLa cells with MP-bC16 in the growth medium at the indicated concentrations. ANOVA statistics for this experiment are reported above.

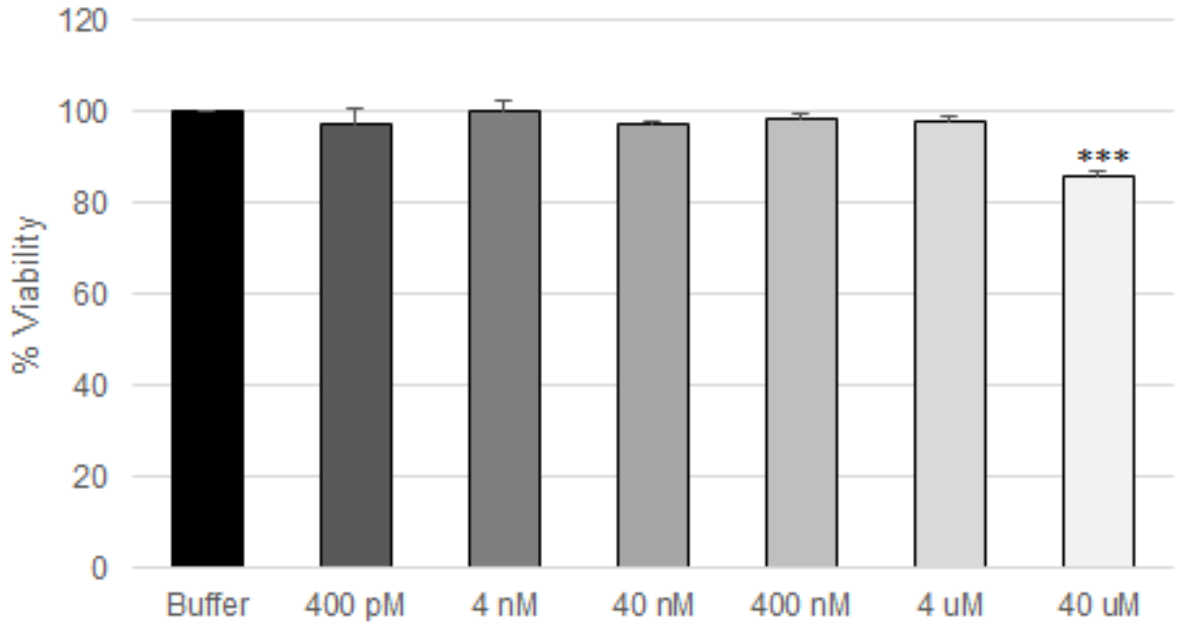

Figure S23: Viability of HeLa cells with TG-C16 in the growth medium at the indicated concentrations. ANOVA statistics for this experiment are reported above; conditions marked $* * *$ are significantly different from untreated cells at $P<0.001$. 


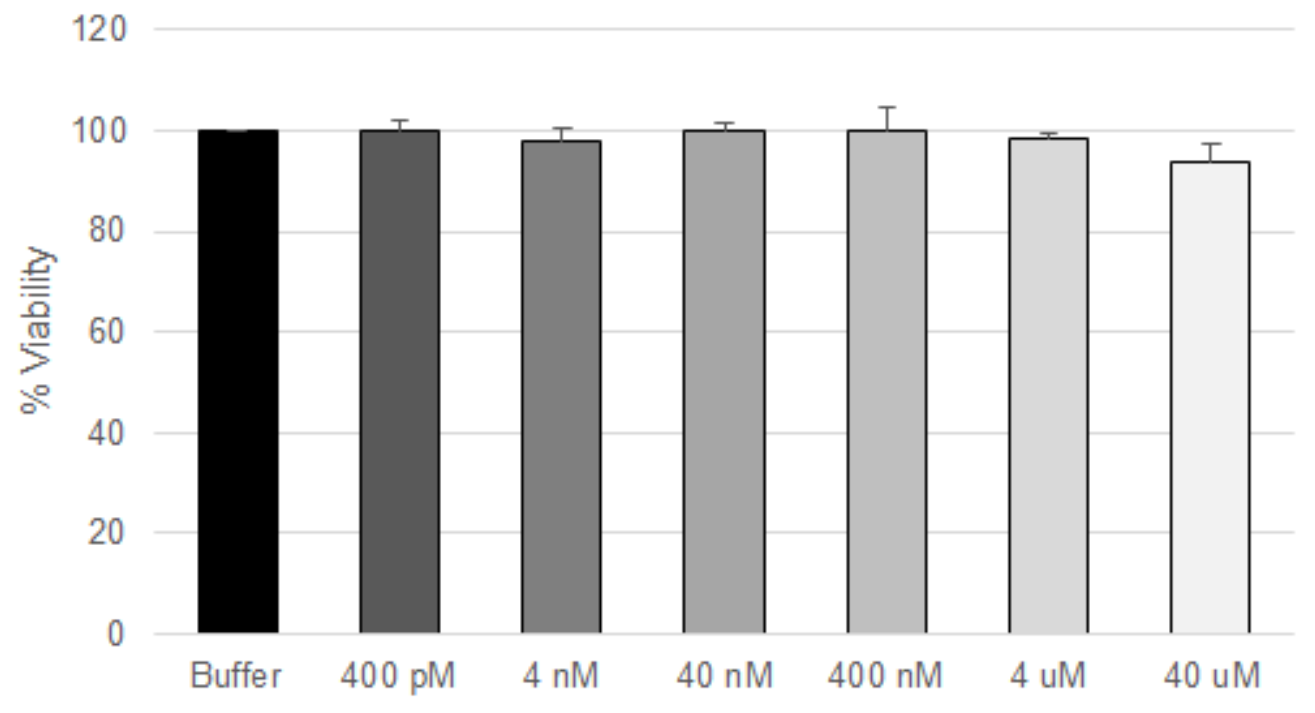

Figure S24: Viability of HeLa cells with TG-C16 in the growth medium at the indicated concentrations. ANOVA statistics for this experiment are reported above.

NMR spectra of compounds prepared 


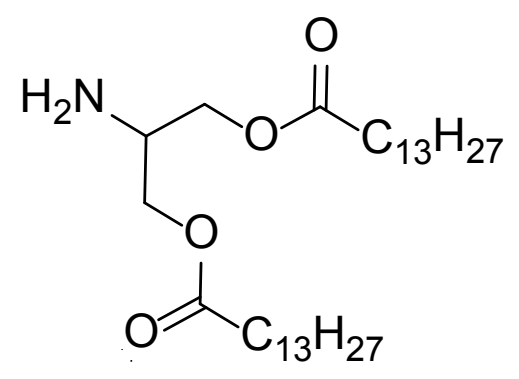

3a ${ }^{1} \mathrm{H}$ NMR $\left(\mathrm{CDCl}_{3}\right) 300 \mathrm{MHz}$

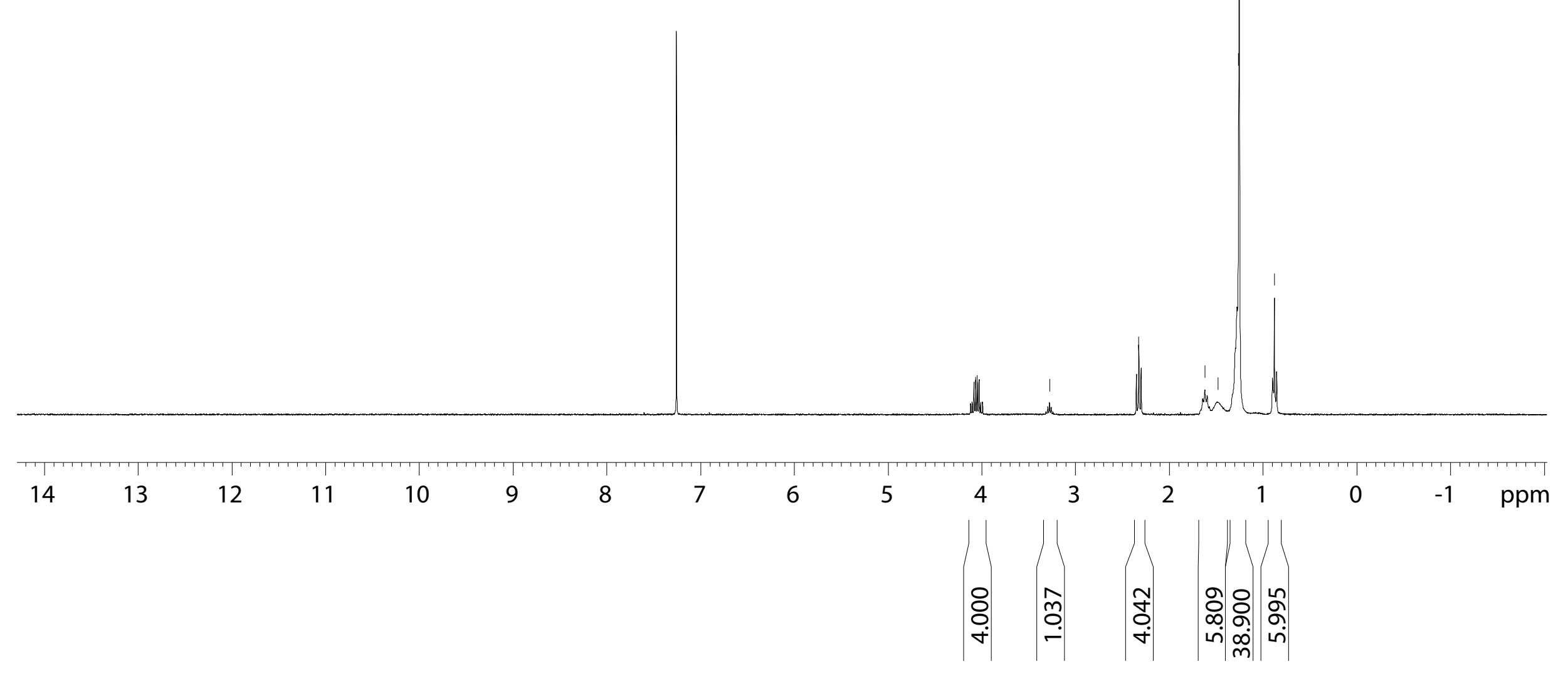




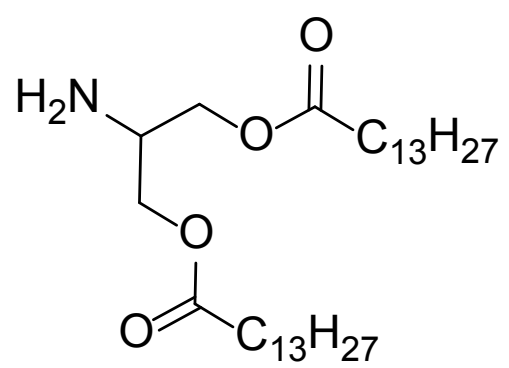

$3 a^{13} \mathrm{C} \mathrm{NMR}\left(\mathrm{CDCl}_{3}\right) 75 \mathrm{MHz}$ 


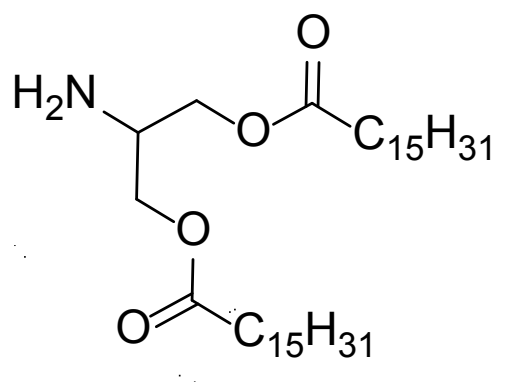

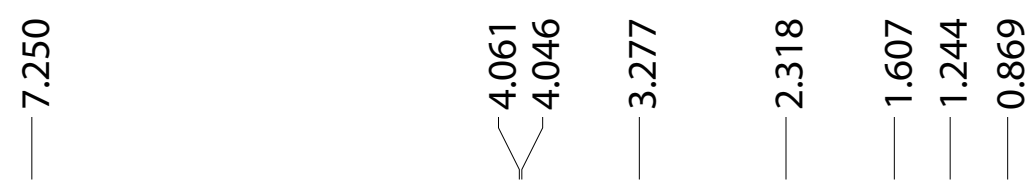

3b ${ }^{1} \mathrm{H}$ NMR $\left(\mathrm{CDCl}_{3}\right) 300 \mathrm{MHz}$

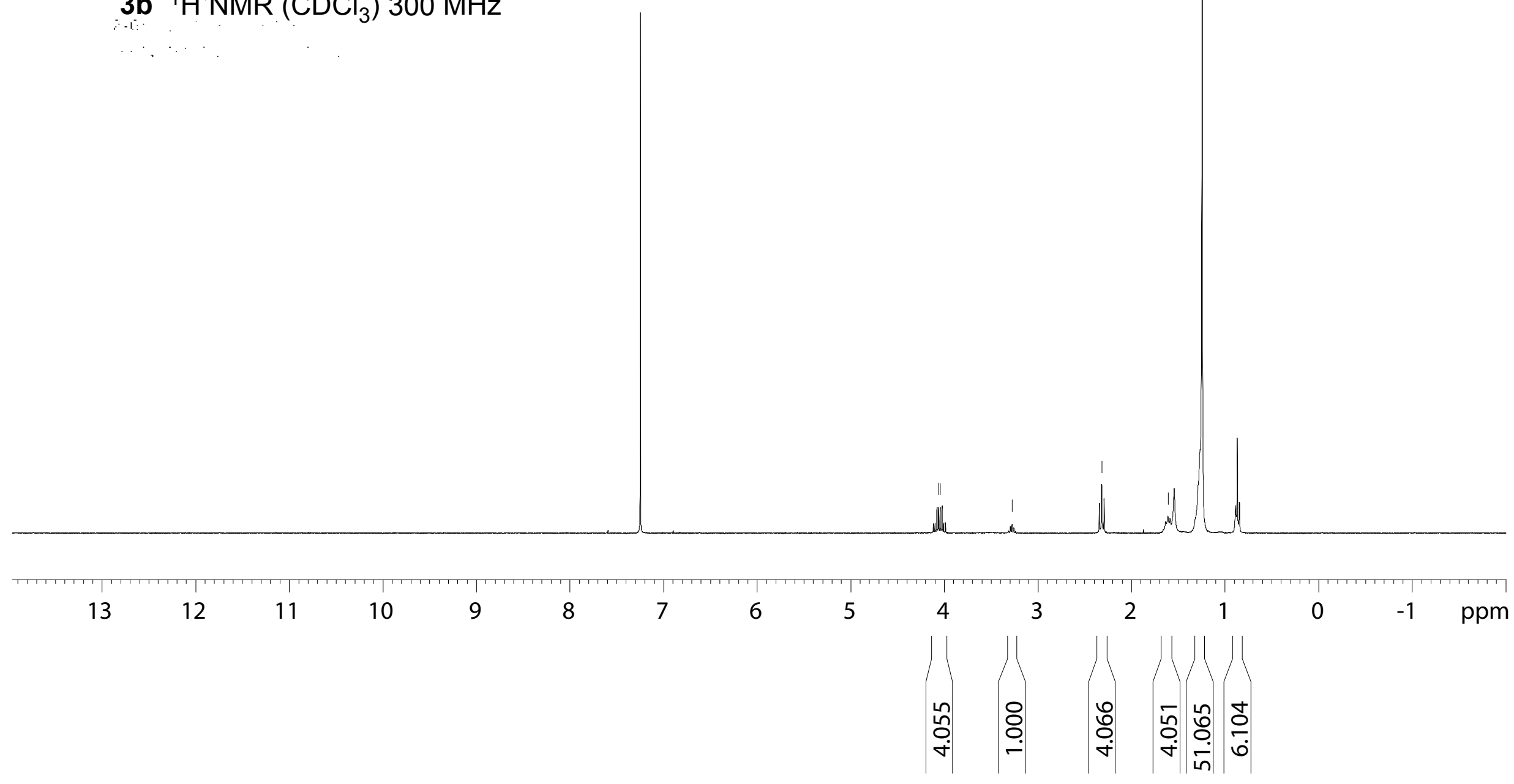




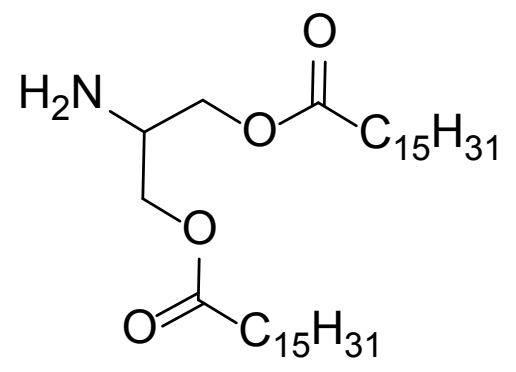

3b ${ }^{13} \mathrm{C} \mathrm{NMR}\left(\mathrm{CDCl}_{3}\right) 75 \mathrm{MHz}$ 


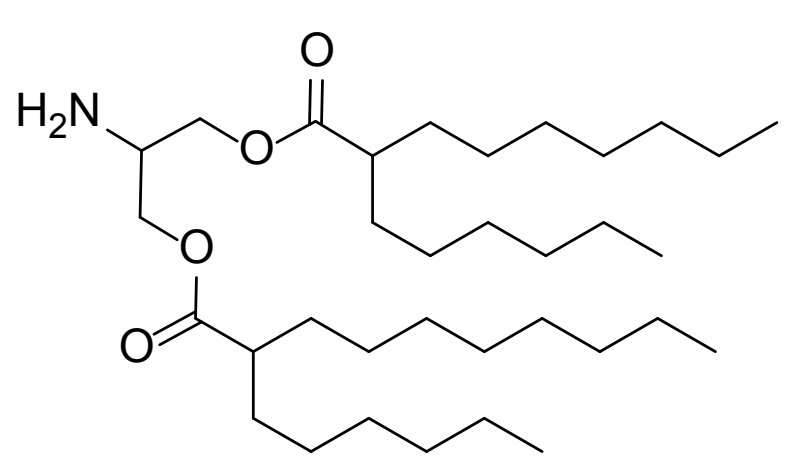

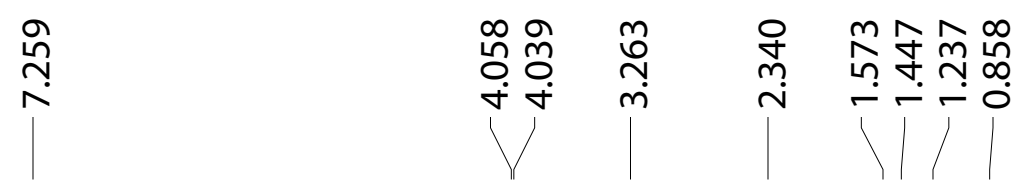

3c ${ }^{1} \mathrm{H}$ NMR $\left(\mathrm{CDCl}_{3}\right) 300 \mathrm{MHz}$

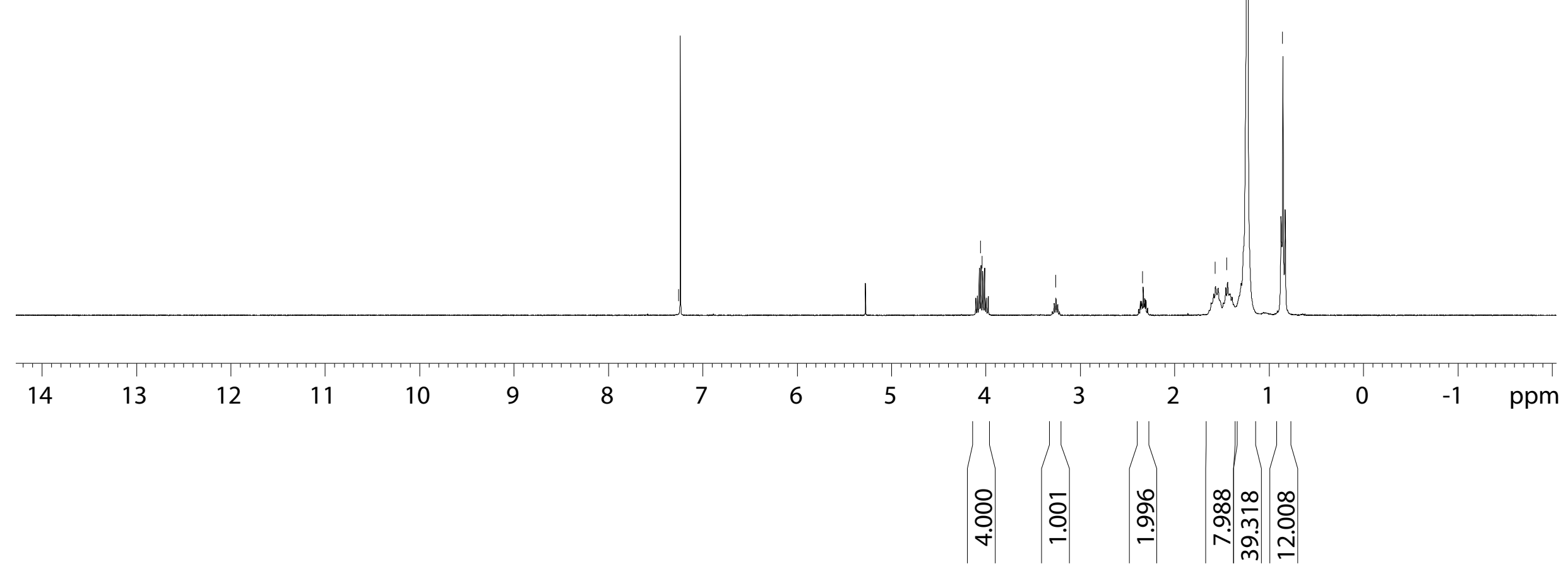




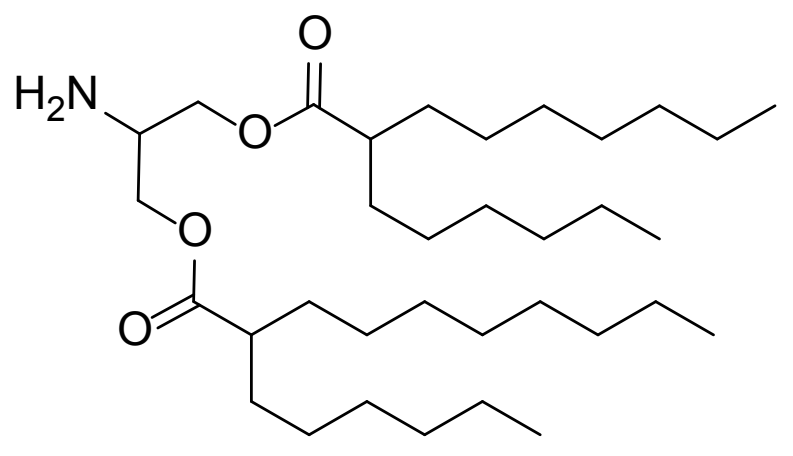

3c ${ }^{13} \mathrm{C} \mathrm{NMR}\left(\mathrm{CDCl}_{3}\right) \quad 75 \mathrm{MHz}$ 


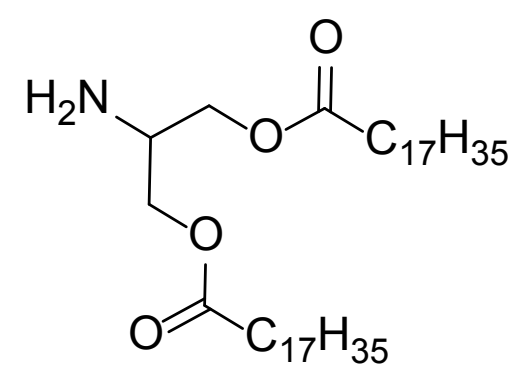

|

3d ${ }^{1} \mathrm{H}$ NMR $\left(\mathrm{CDCl}_{3}\right) 300 \mathrm{MHz}$

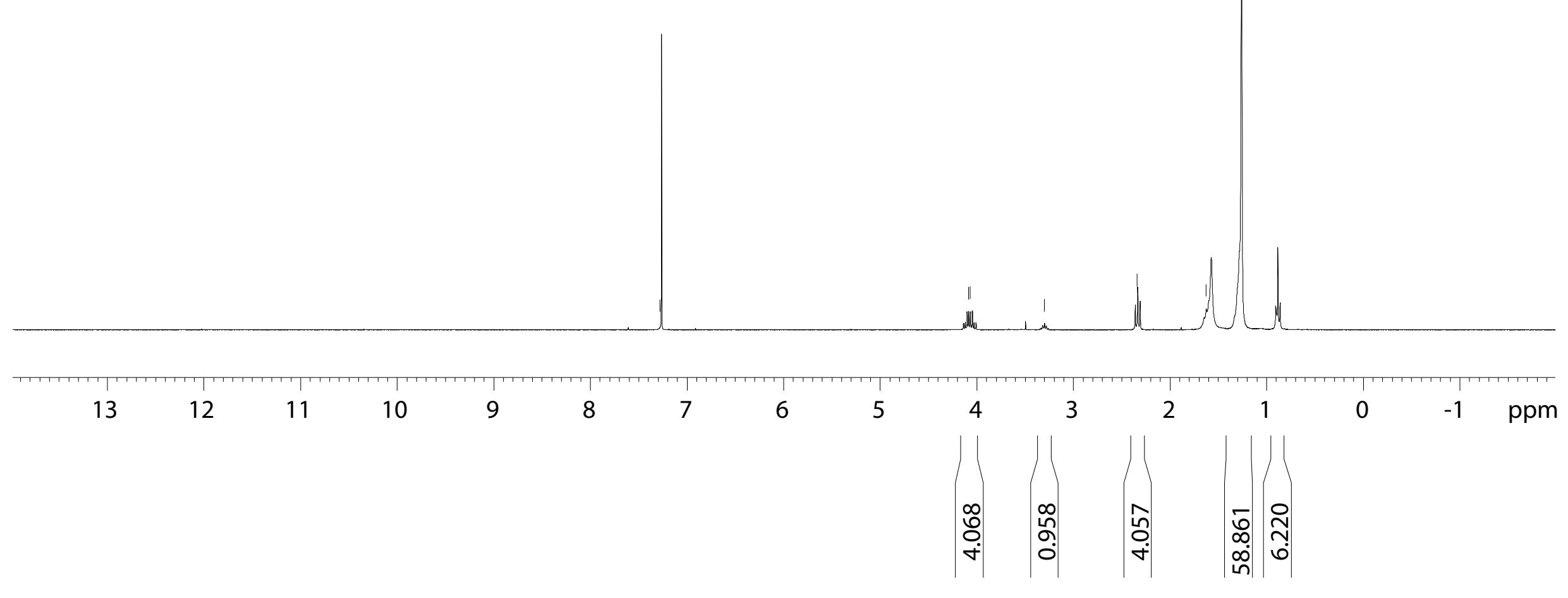


<smiles>[CH+]C(=O)OCC(N)COC(=O)C(F)(F)F</smiles>

3d ${ }^{13} \mathrm{C} \mathrm{NMR}\left(\mathrm{CDCl}_{3}\right) 75 \mathrm{MHz}$ 


\begin{tabular}{|c|c|c|c|c|c|}
\hline-6 & の & $\infty$ & $\digamma$ & \llcorner & $\infty$ \\
\hline 6 - & 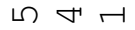 & $\sigma$ & - & $\infty$ & $\nabla$ \\
\hline N 0 & $\nabla \curvearrowright r$ & $\sim$ & $m$ & 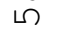 & $\sim$ \\
\hline$\dot{r}$ & $\dot{\nabla} \dot{\nabla} \dot{\gamma}$ & $\dot{m}$ & $\dot{\sim}$ & -1 & $\dot{r}$ \\
\hline
\end{tabular}

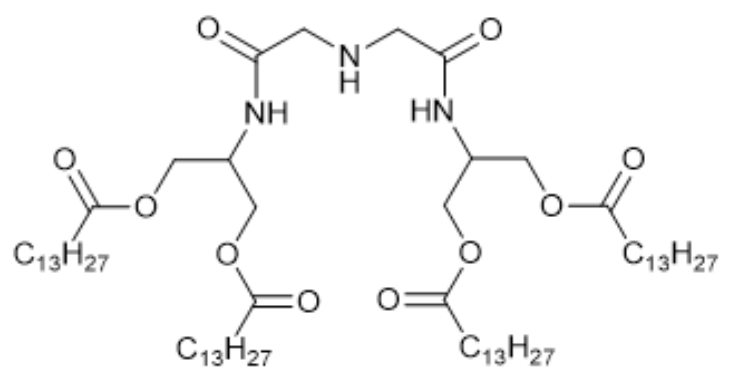

5a ${ }^{1} \mathrm{H} \mathrm{NMR}\left(\mathrm{CDCl}_{3}\right) 300 \mathrm{MHz}$

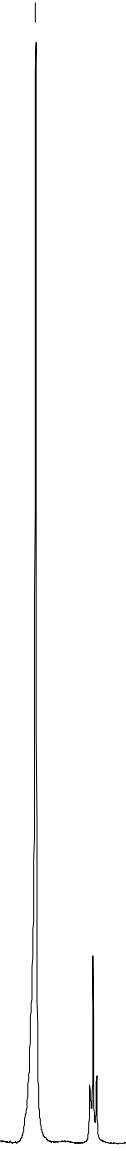

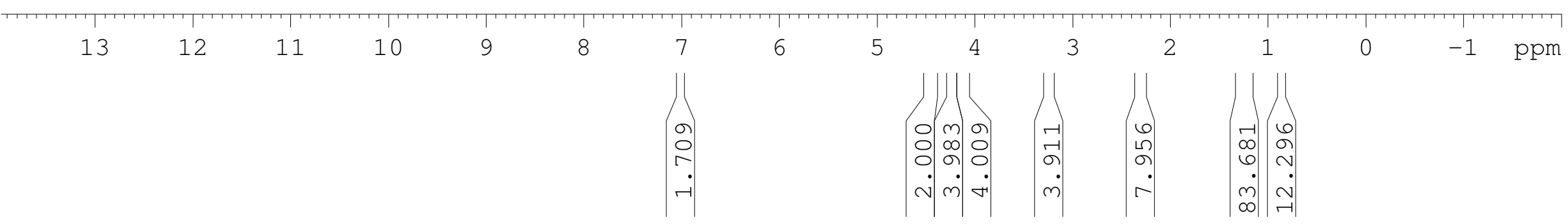




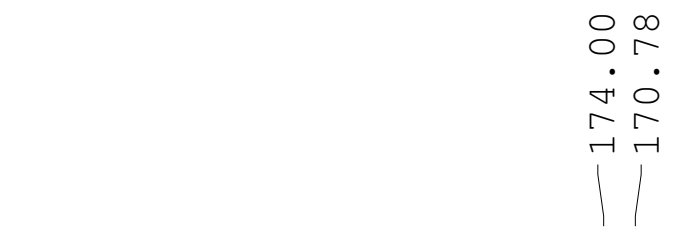

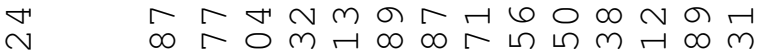

$\therefore \quad \dot{\sim} \infty \dot{\sim} \dot{\sim} \dot{\sigma} \dot{\sigma} \dot{\sigma} \dot{\sigma} \dot{\sigma} \dot{\sim} \dot{\sim}$

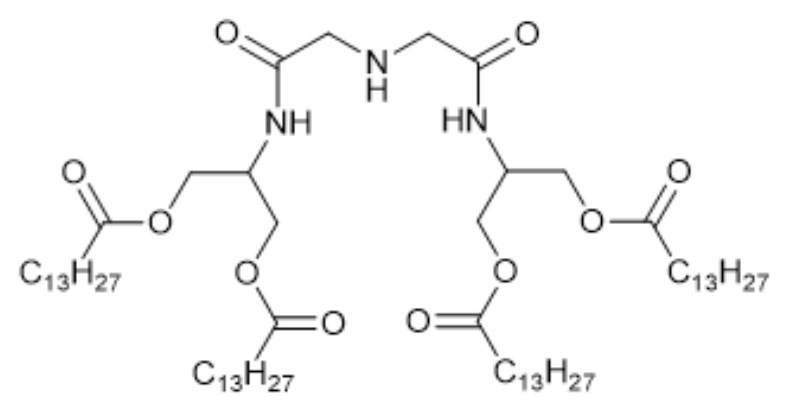

$5 \mathbf{a}^{13} \mathrm{C} \mathrm{NMR}\left(\mathrm{CDCl}_{3}\right) 75 \mathrm{MHz}$

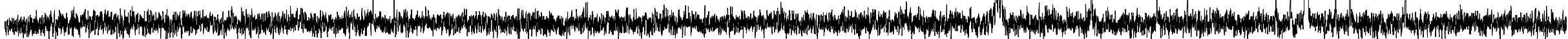

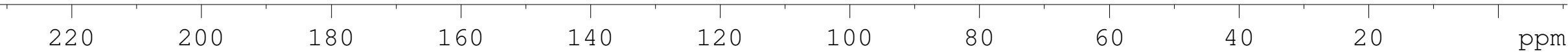




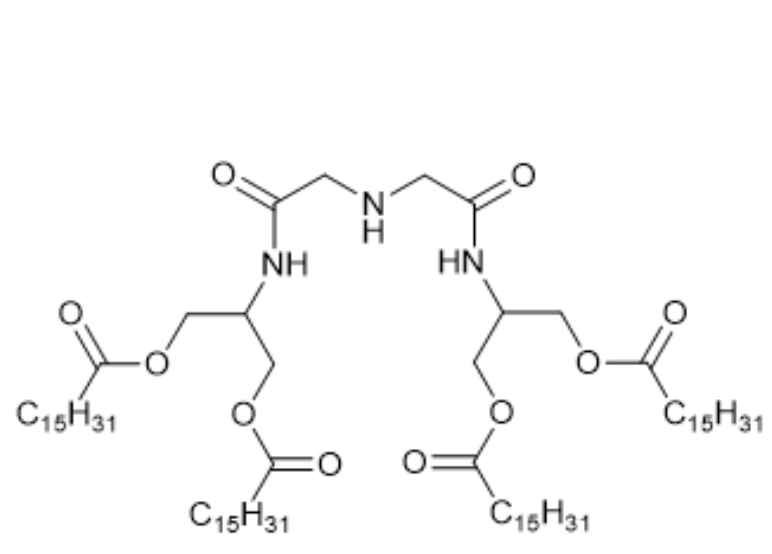

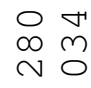

$m \sim r$

$\pi \sigma^{2}$,

r.

అ $\begin{array}{lllll}m & m & 0 & 0\end{array}$

$\sim r$

. $\$.

5b ${ }^{1} \mathrm{H}$ NMR $\left(\mathrm{CDCl}_{3}\right) 300 \mathrm{MHz}$

\section{5b $1 \mathrm{H} N \mathrm{NR}(\mathrm{CDCl}) 300 \mathrm{MHZ}$}

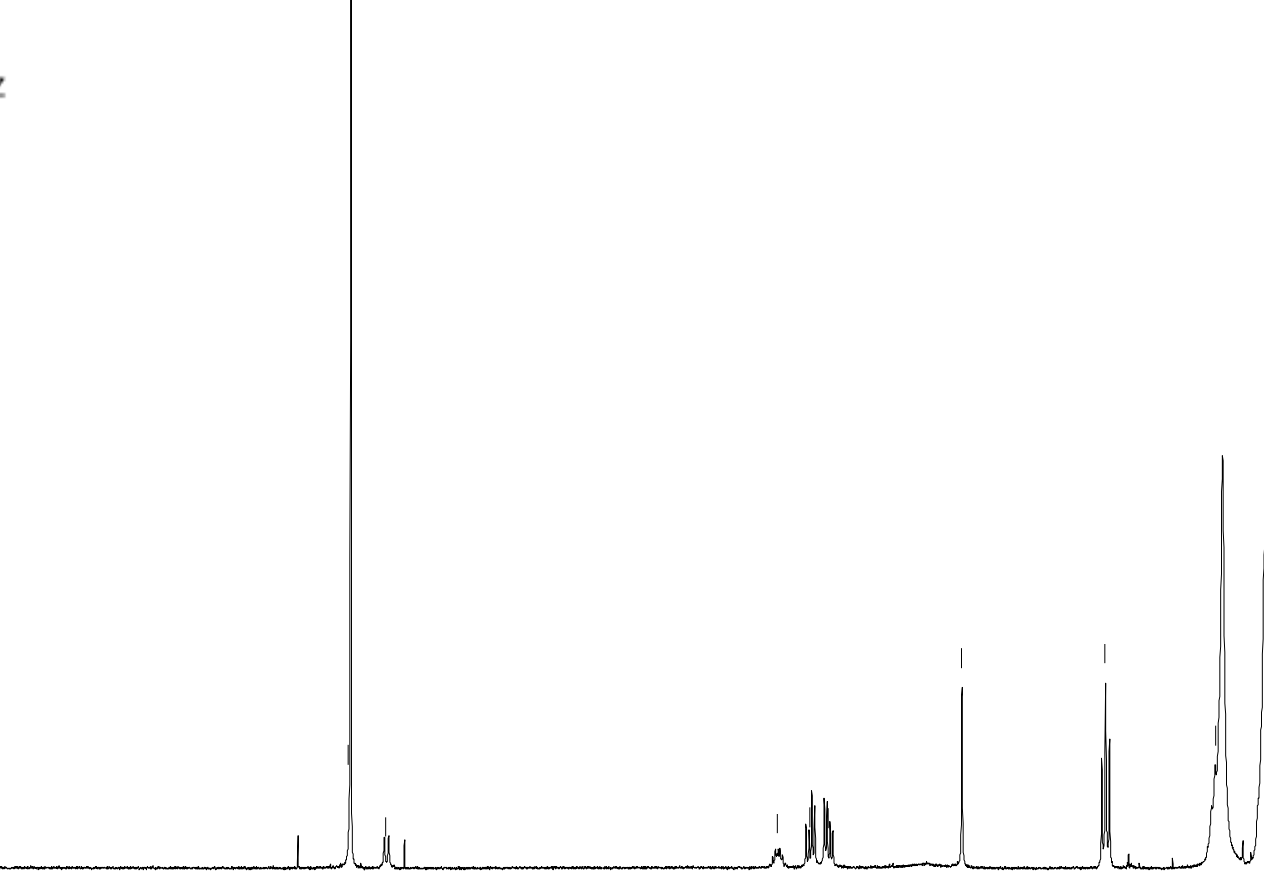

V

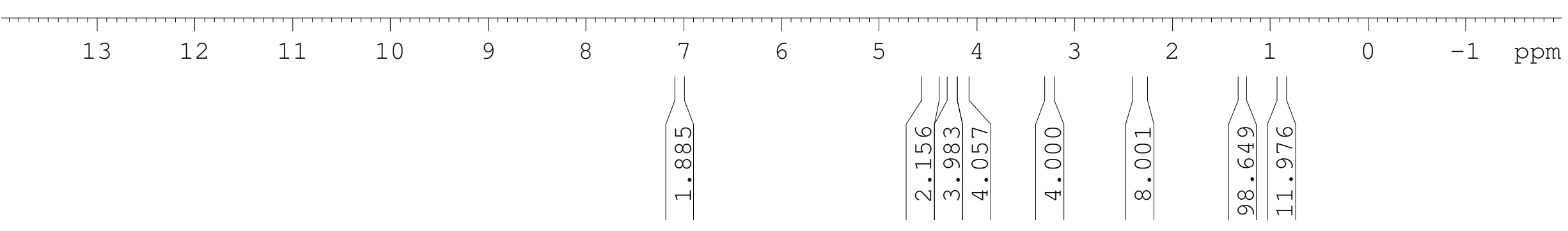




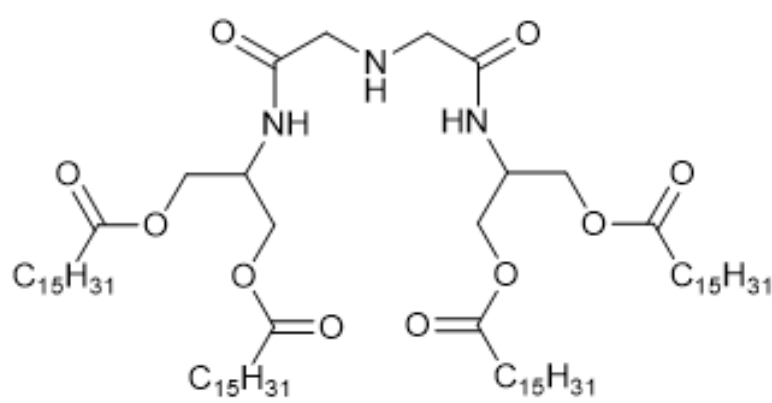

5b ${ }^{13} \mathrm{C} \mathrm{NMR}\left(\mathrm{CDCl}_{3}\right) 75 \mathrm{MHz}$

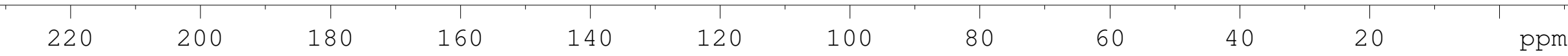




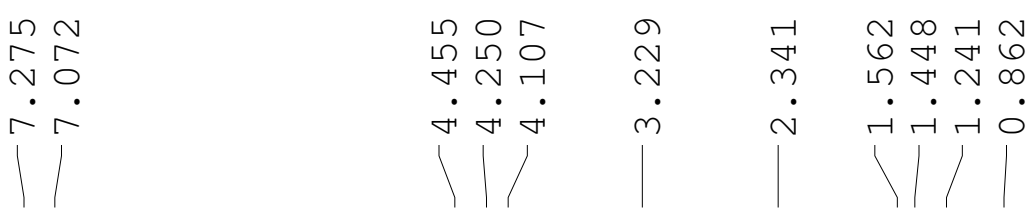

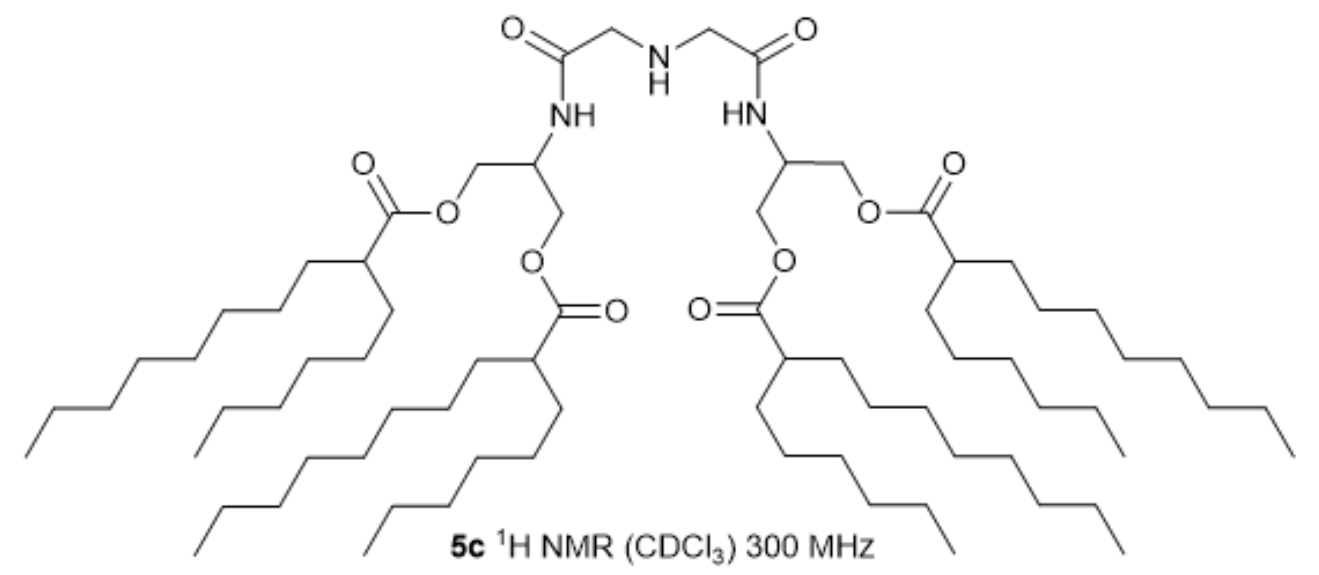

I ith M.

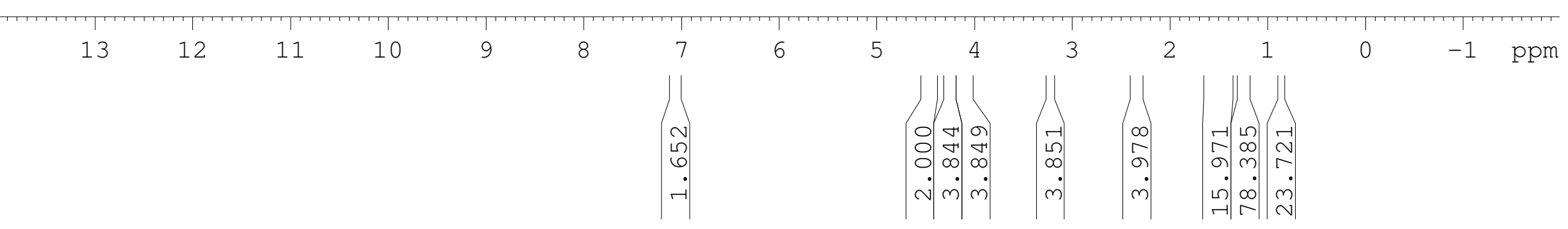



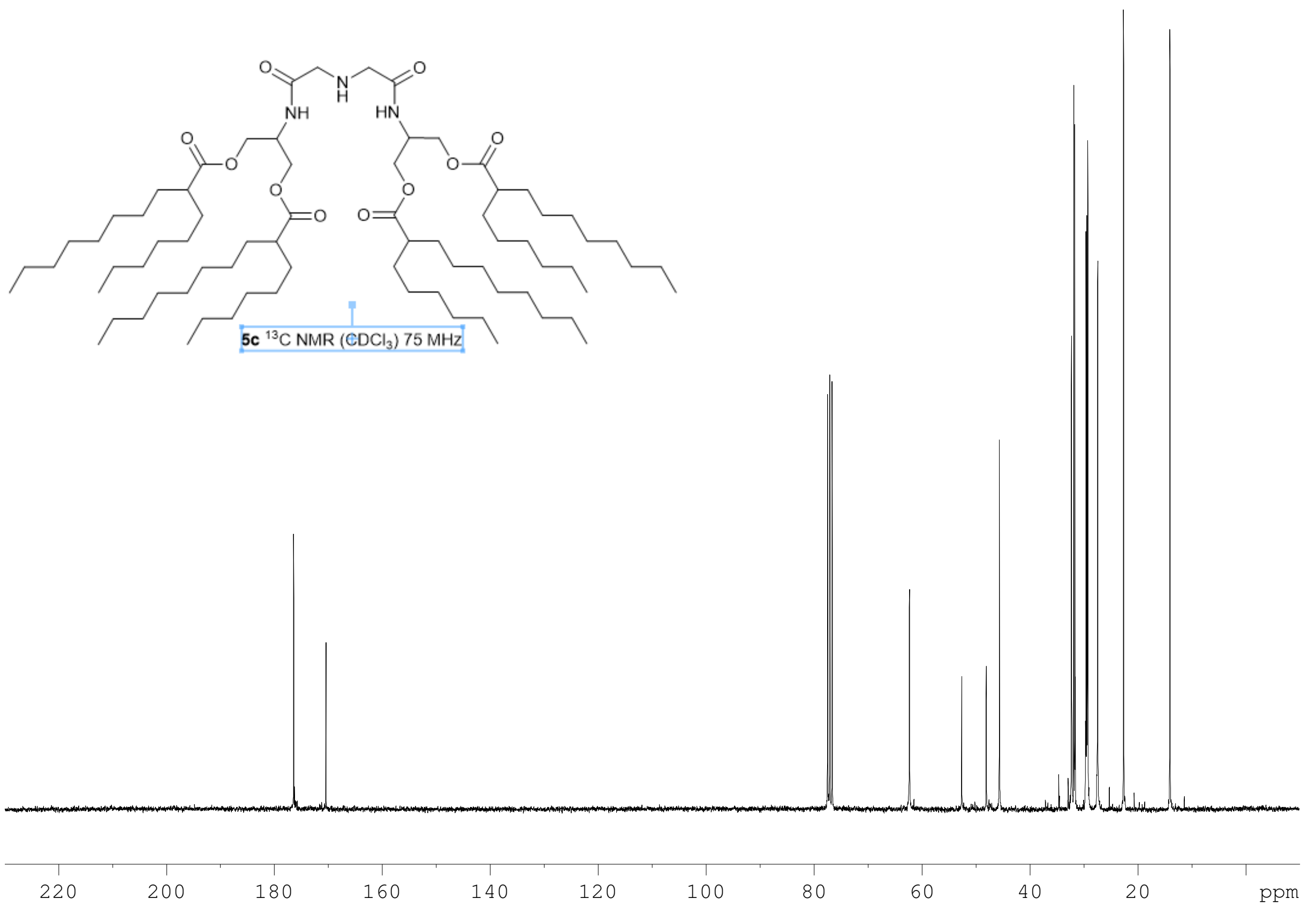


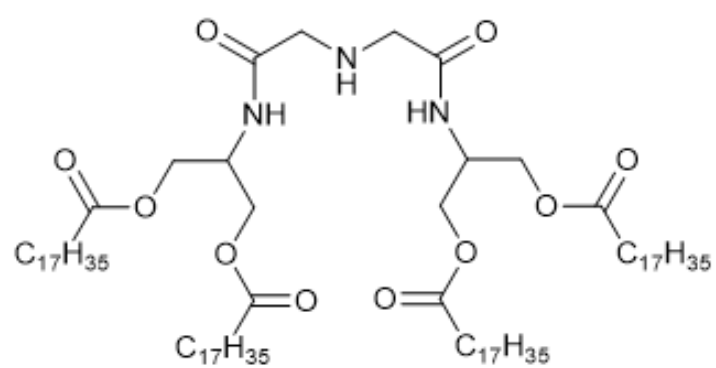

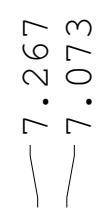

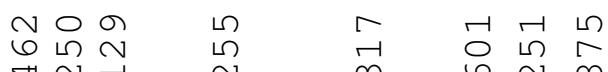

.

अं वं

-

5d ${ }^{1} \mathrm{H}$ NMR $\left(\mathrm{CDCl}_{3}\right) 300 \mathrm{MHz}$

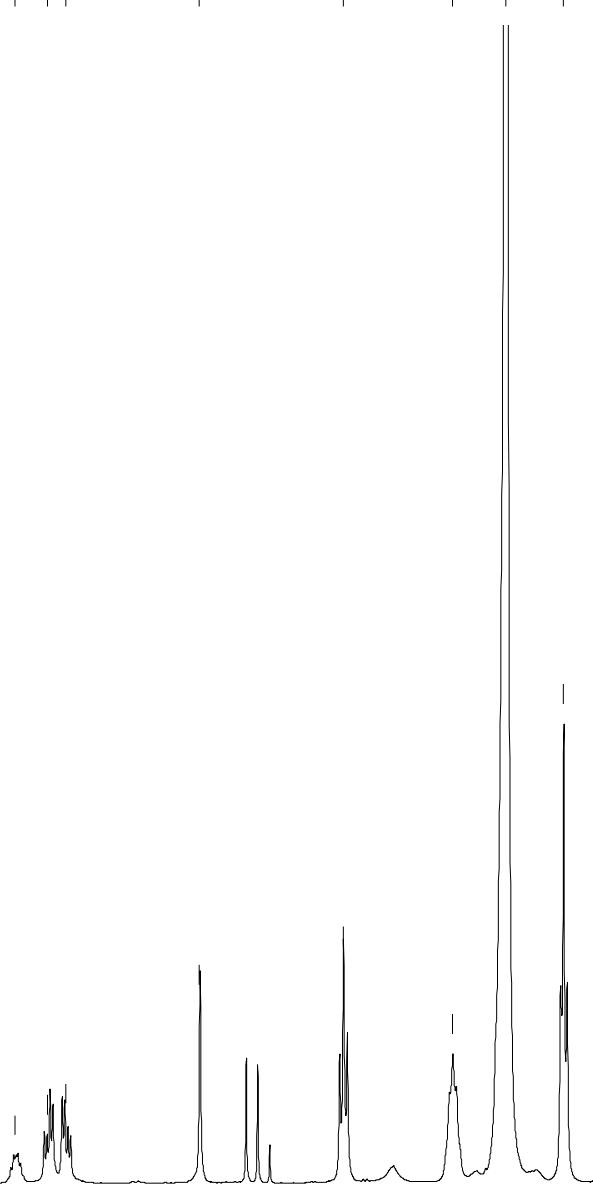

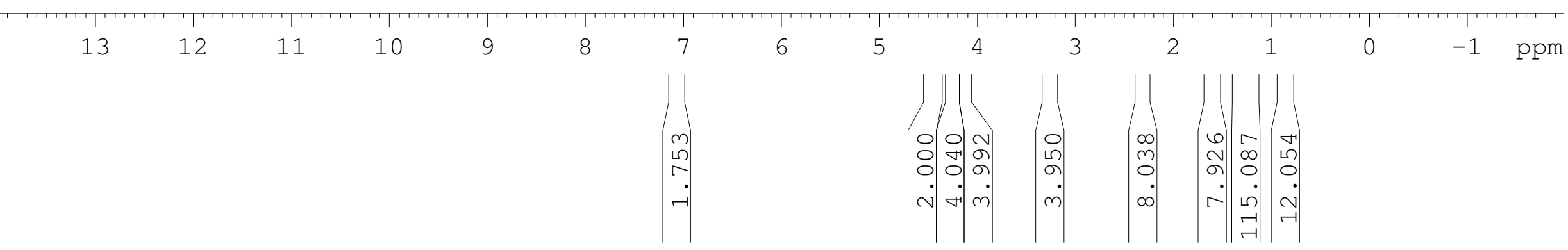




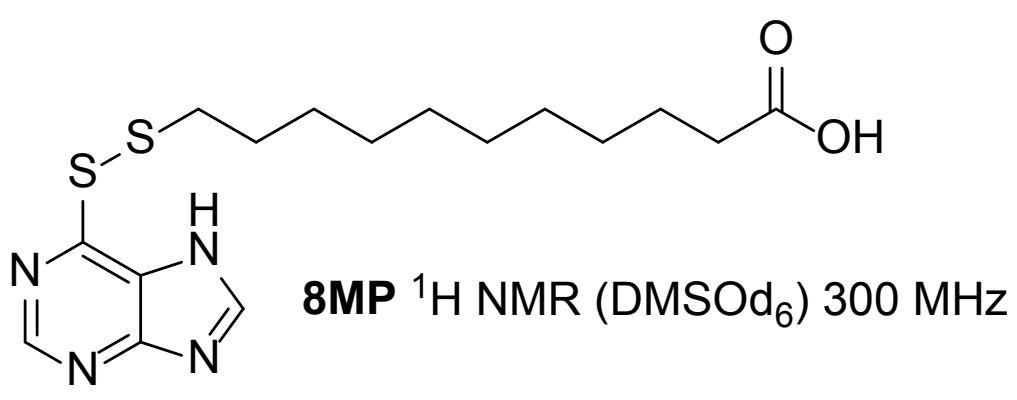


<smiles>O=C(O)CCCCCCCCCCSSc1ncnc2nc[nH]c12</smiles> 


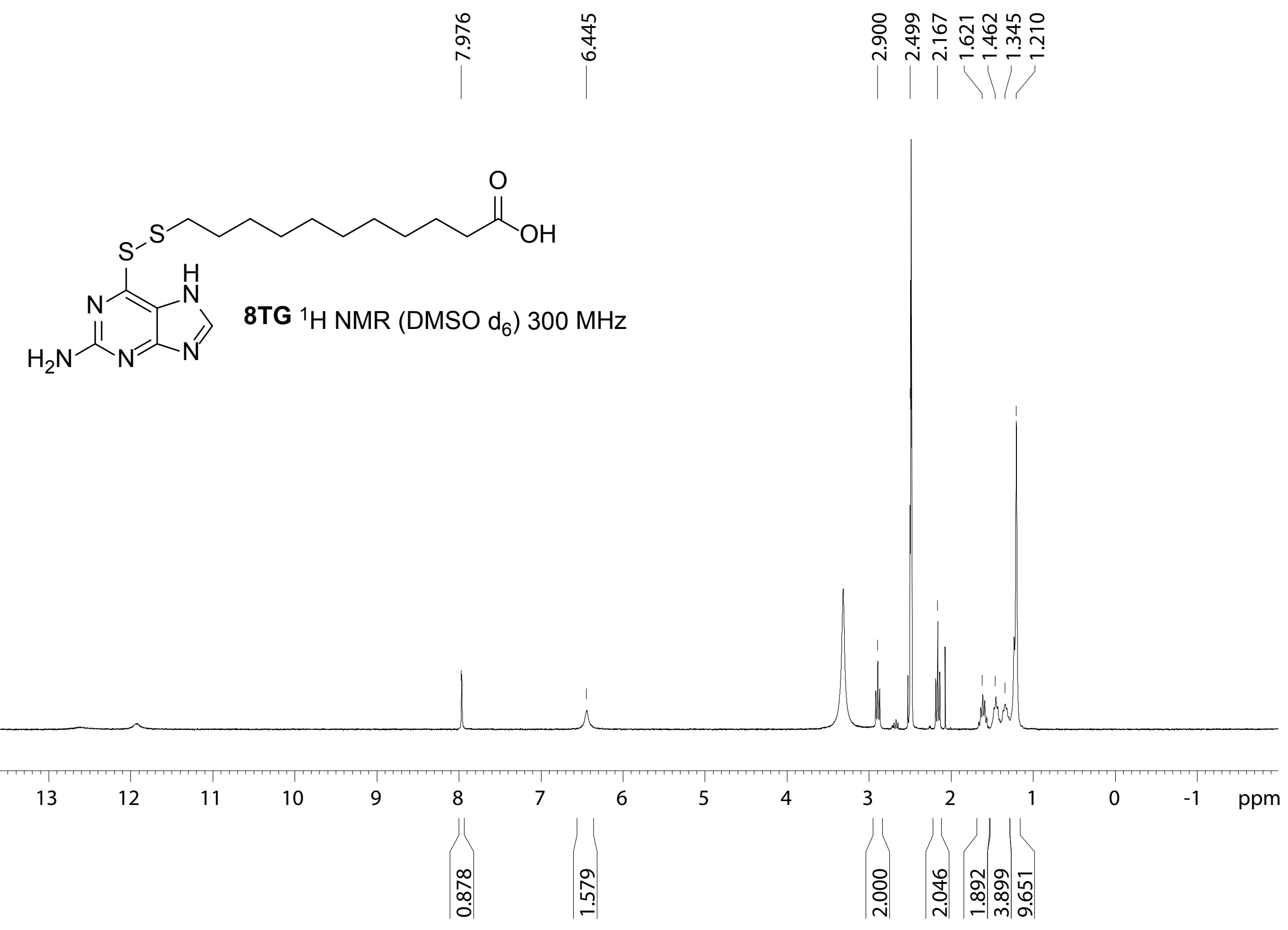




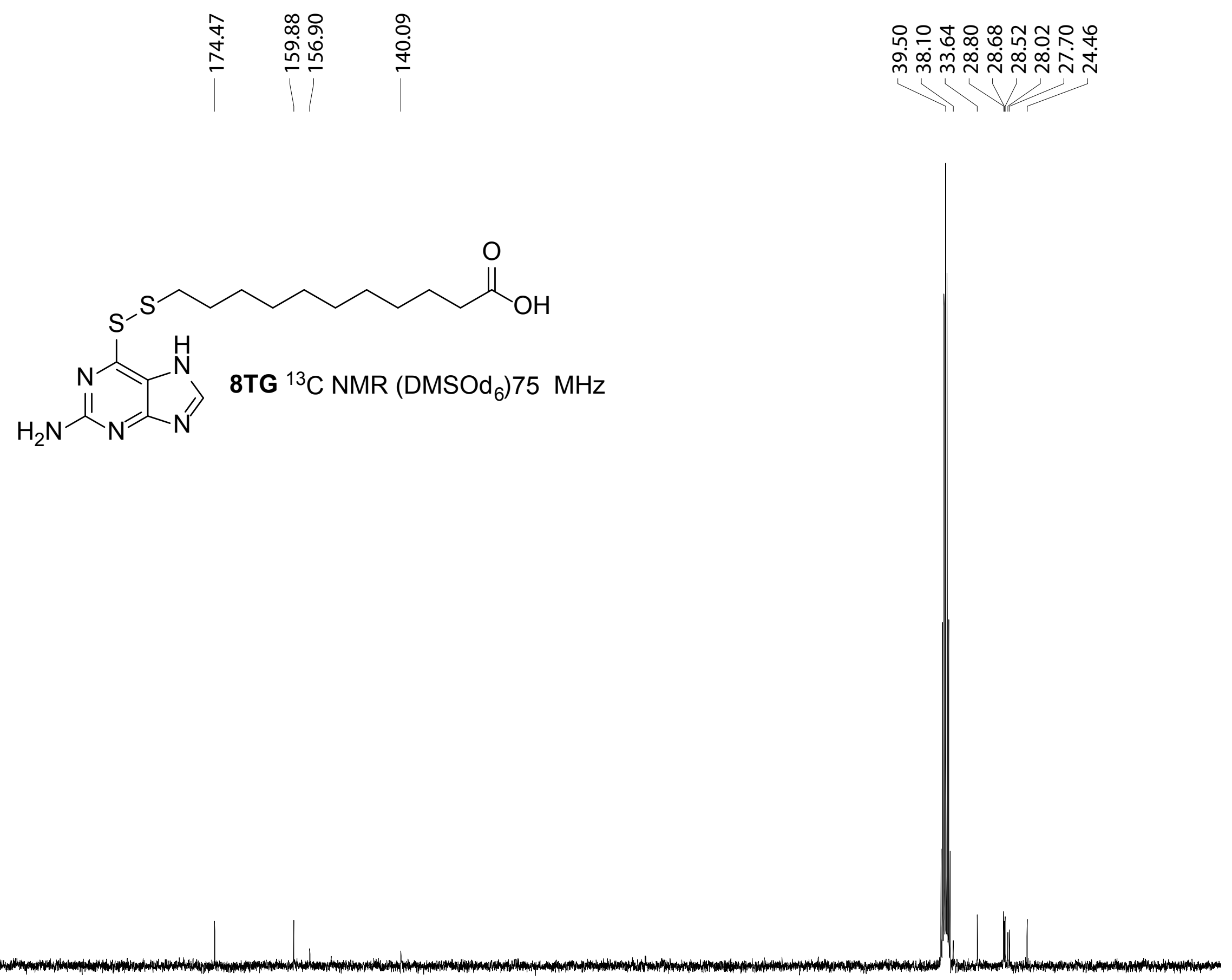



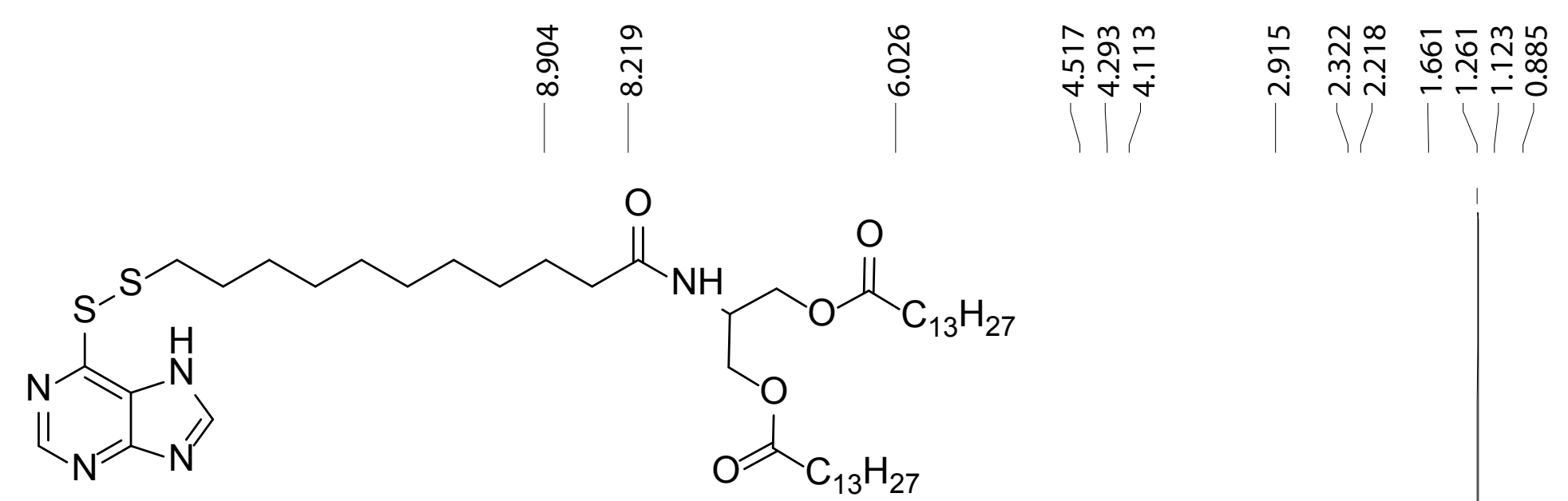

MP-C14 ${ }^{1} \mathrm{H} \mathrm{NMR}\left(\mathrm{CDCl}_{3}\right) 300 \mathrm{MHz}$

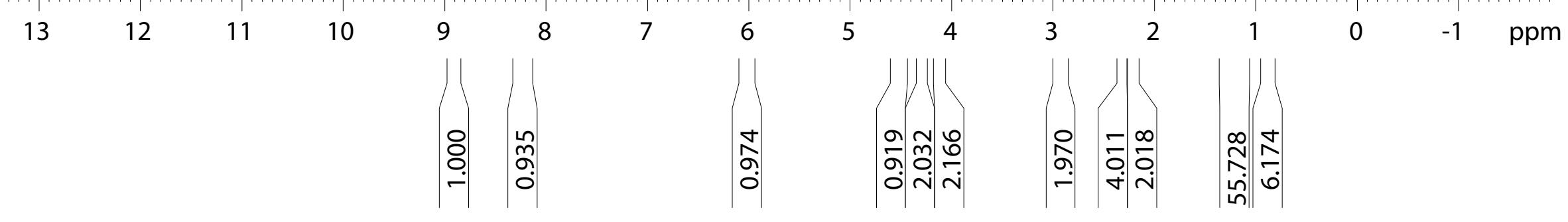



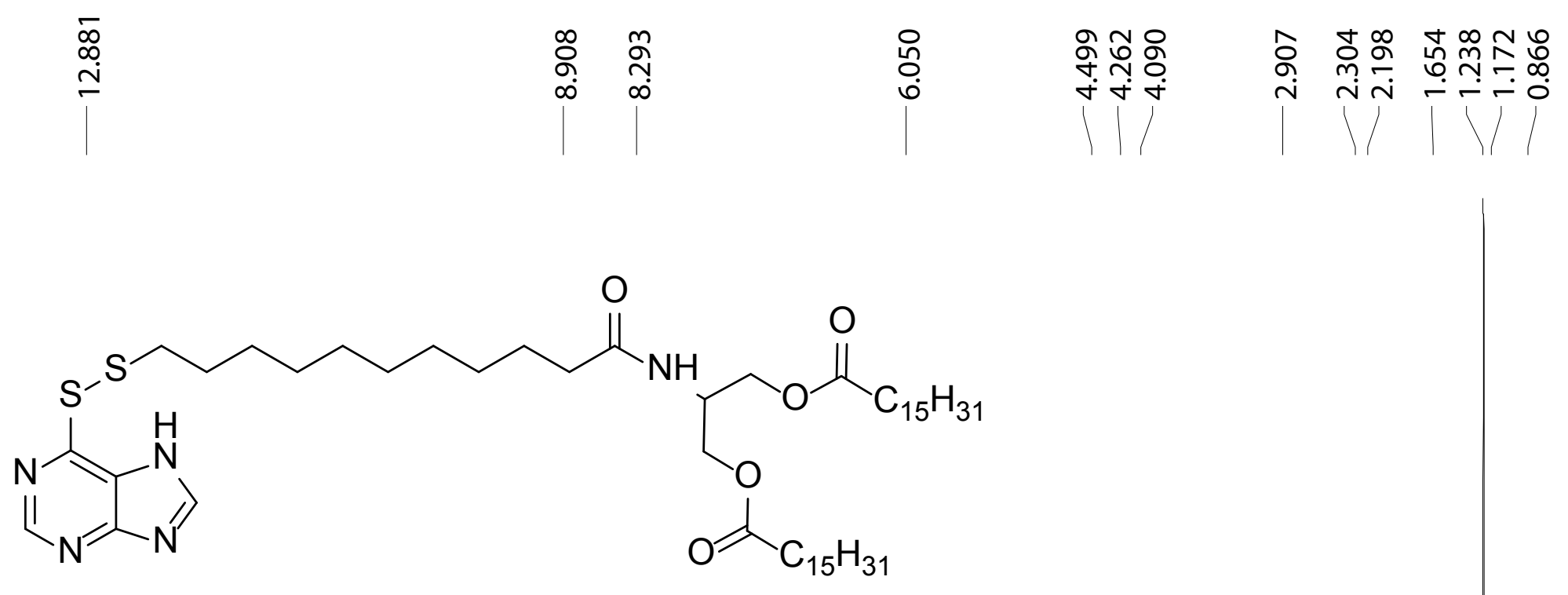

MP-C16 ${ }^{1} \mathrm{H} \mathrm{NMR}\left(\mathrm{CDCl}_{3}\right) 300 \mathrm{MHz}$

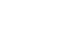




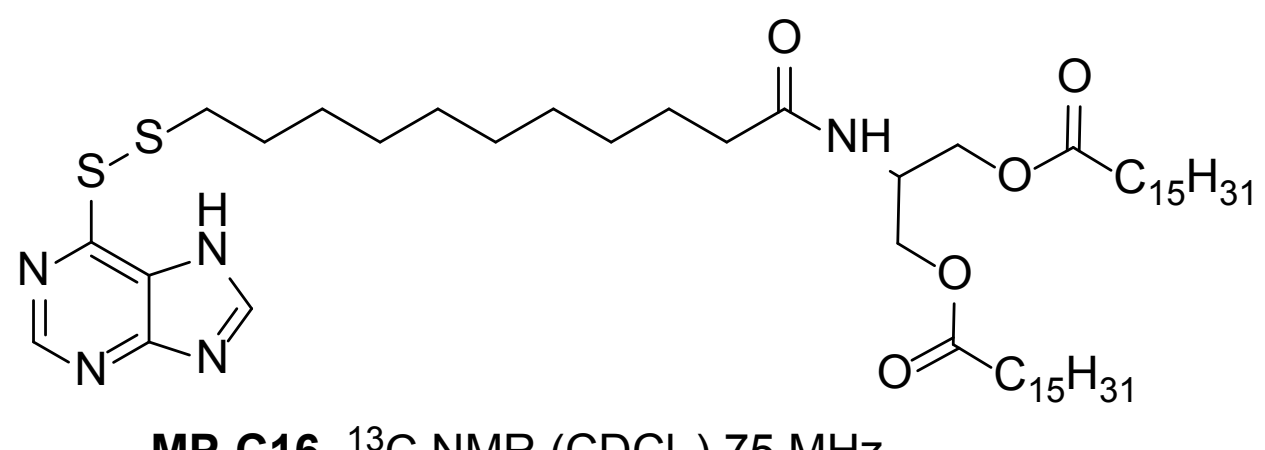

MP-C16 ${ }^{13} \mathrm{C} \mathrm{NMR}\left(\mathrm{CDCl}_{3}\right) 75 \mathrm{MHz}$ 

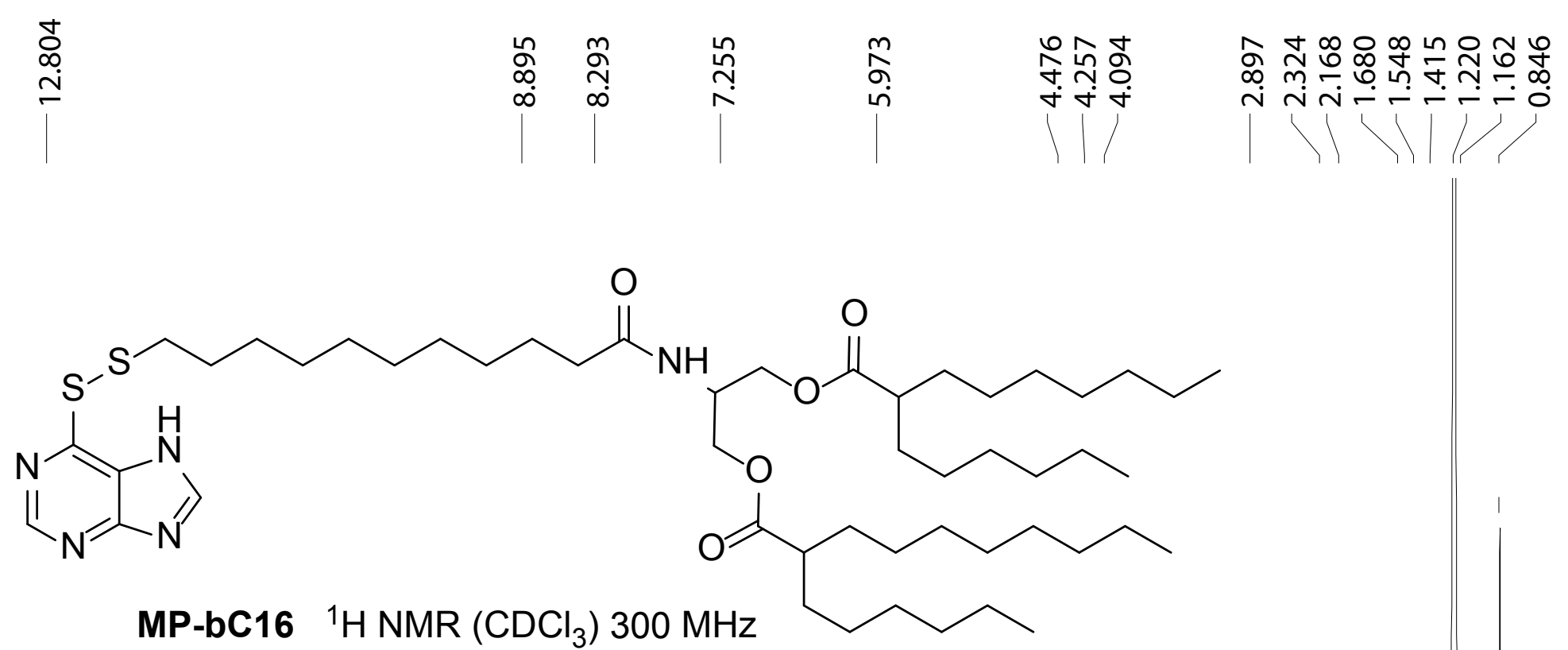

MP-bC16 ${ }^{1} \mathrm{H} \mathrm{NMR}\left(\mathrm{CDCl}_{3}\right) 300 \mathrm{MHz}$

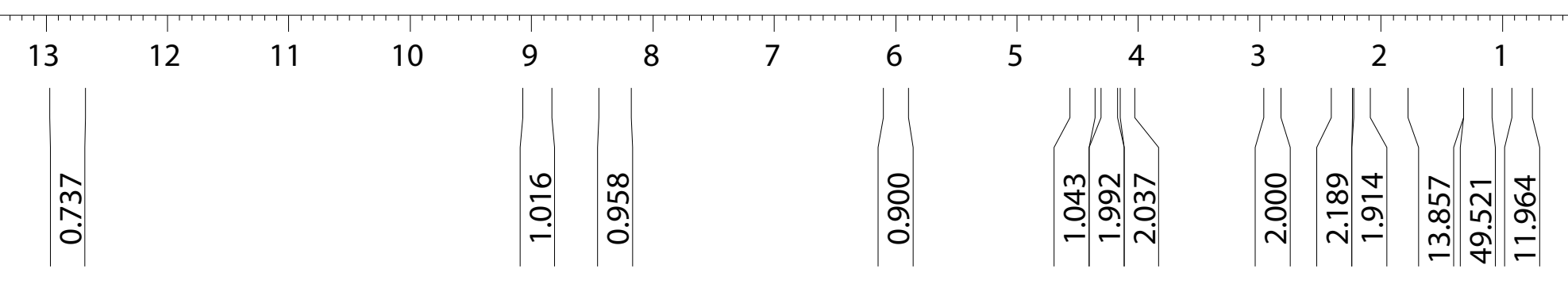



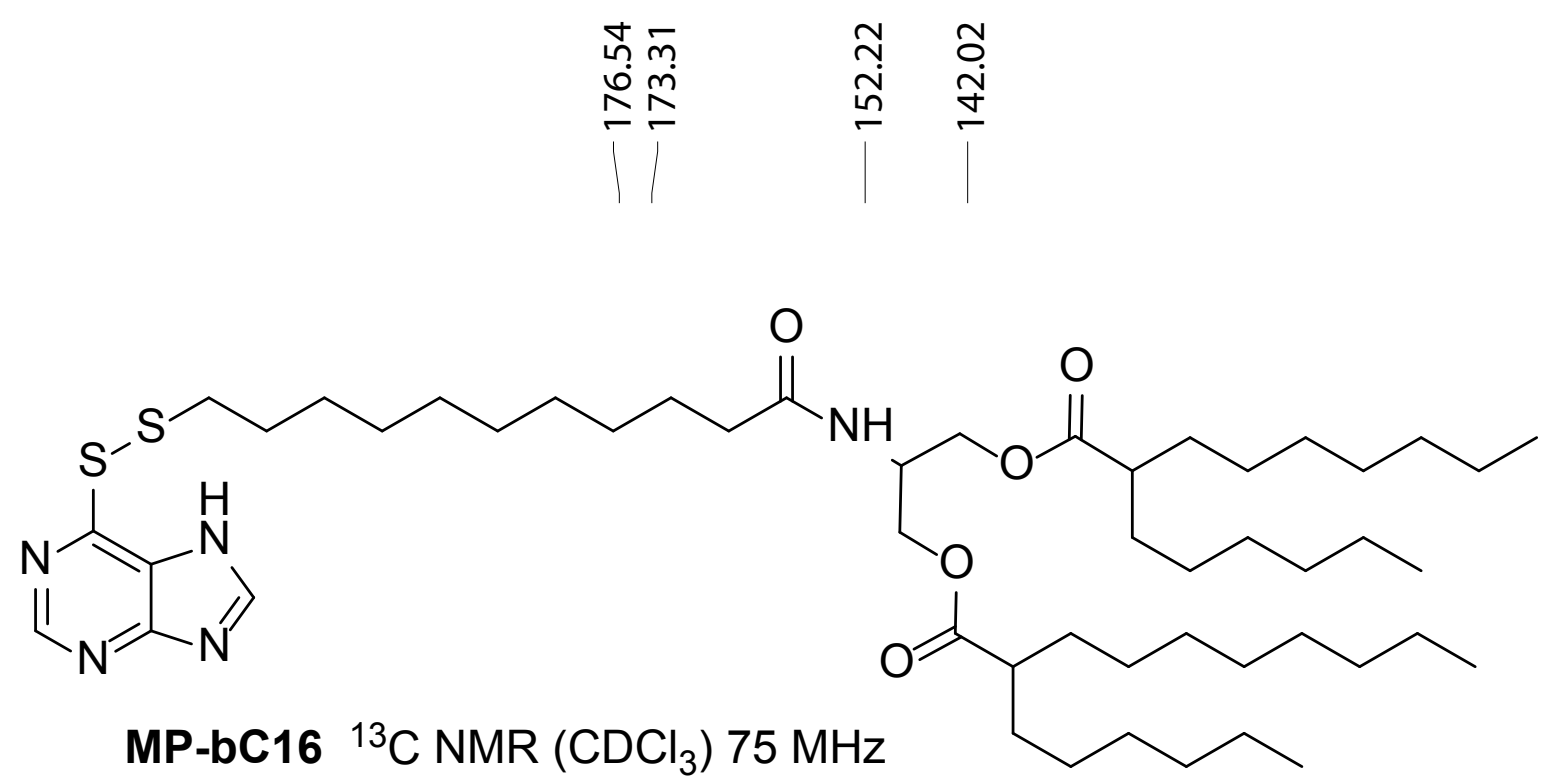

MP-bC16 ${ }^{13} \mathrm{C} \mathrm{NMR}\left(\mathrm{CDCl}_{3}\right) 75 \mathrm{MHz}$

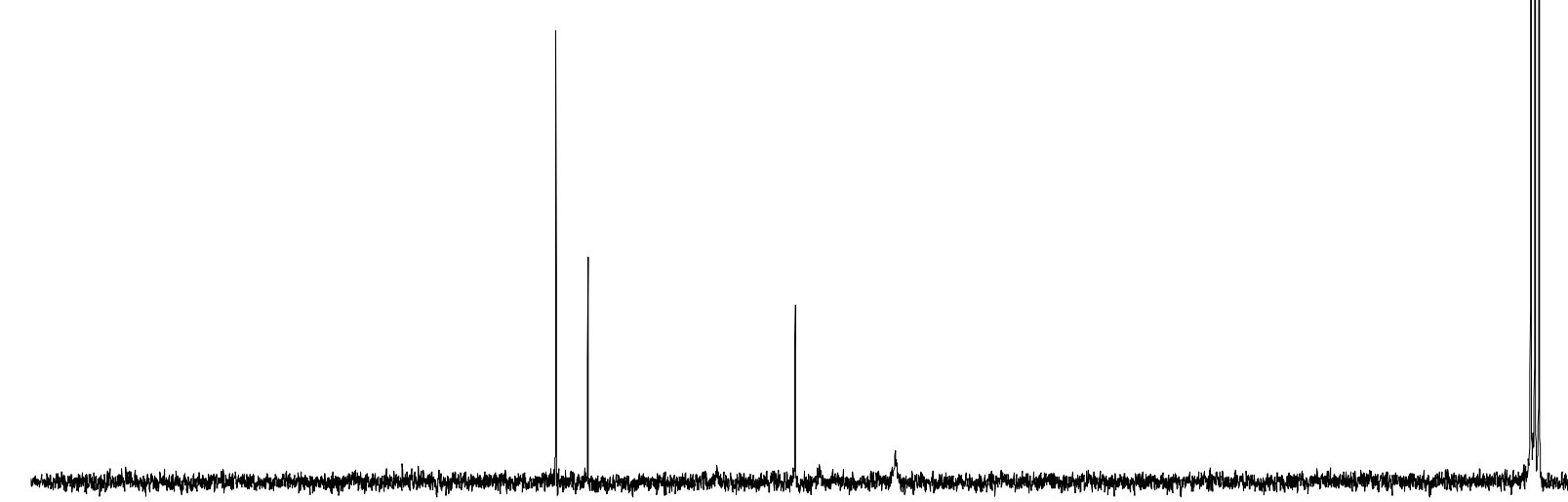



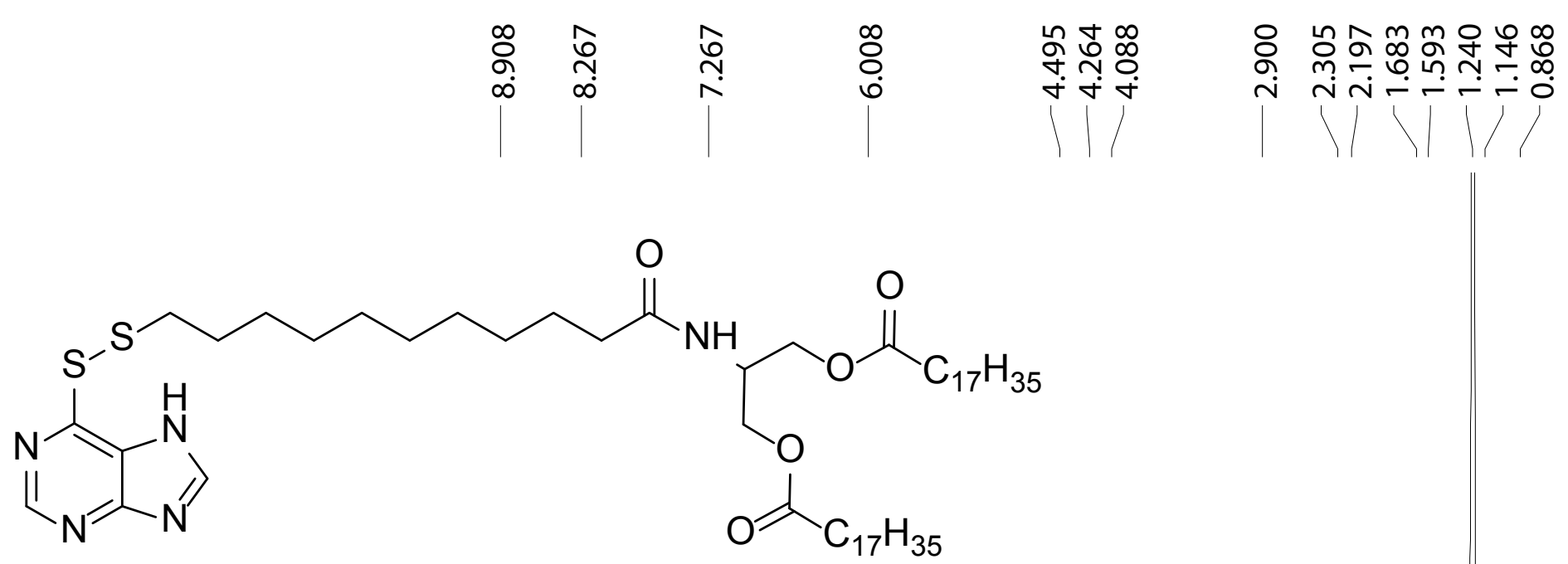

MP-C18 ${ }^{1} \mathrm{H} \mathrm{NMR}\left(\mathrm{CDCl}_{3}\right) 300 \mathrm{MHz}$
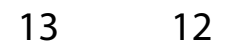

11
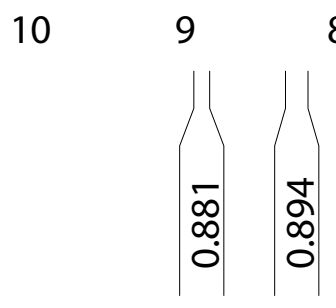

\%

7
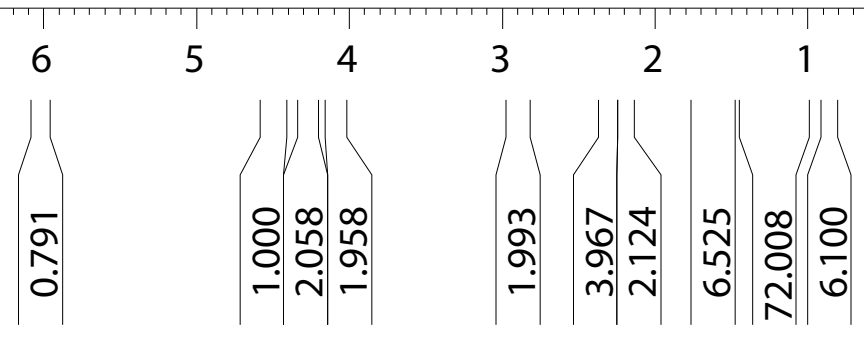

-1 ppm 


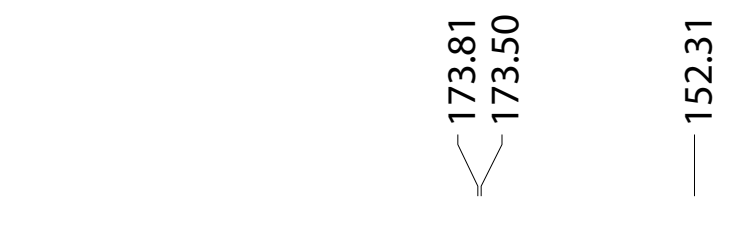

$\frac{n}{n}$

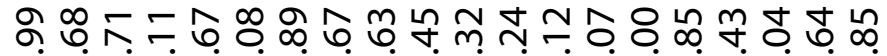

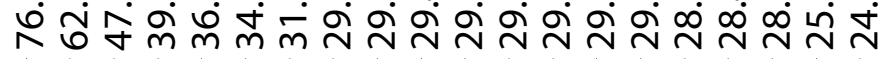

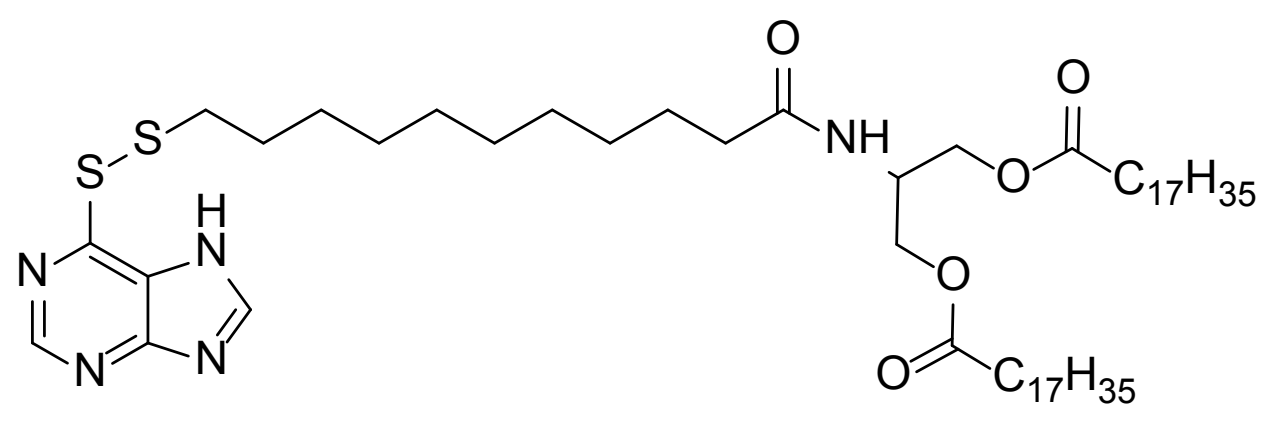

MP-C18 ${ }^{13} \mathrm{C} \mathrm{NMR}\left(\mathrm{CDCl}_{3}\right) 75 \mathrm{MHz}$ 


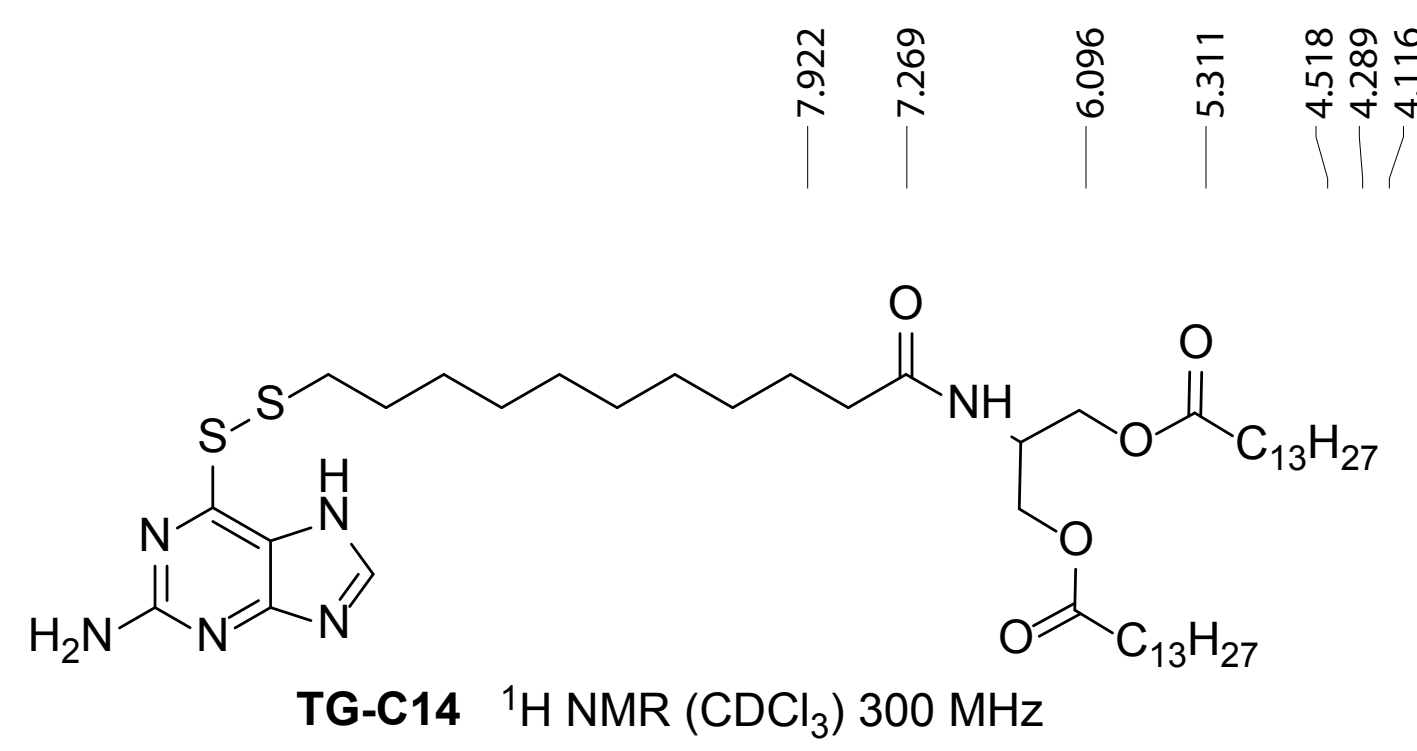

冓

$-\div$

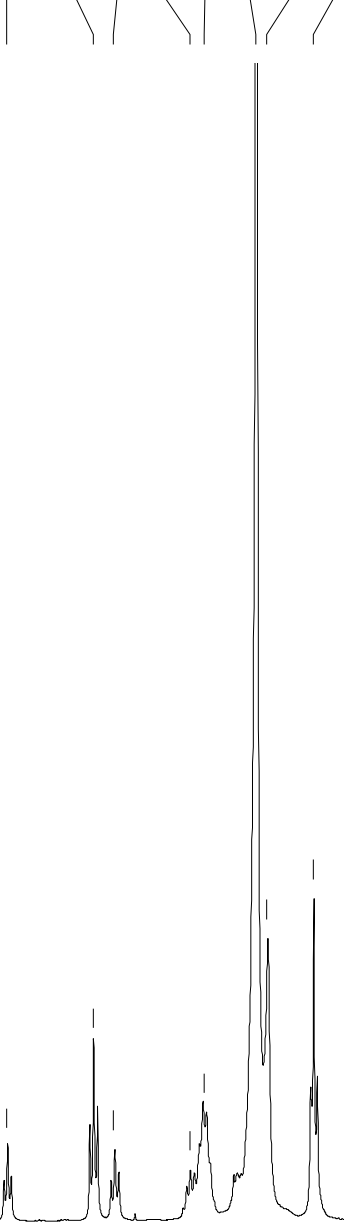

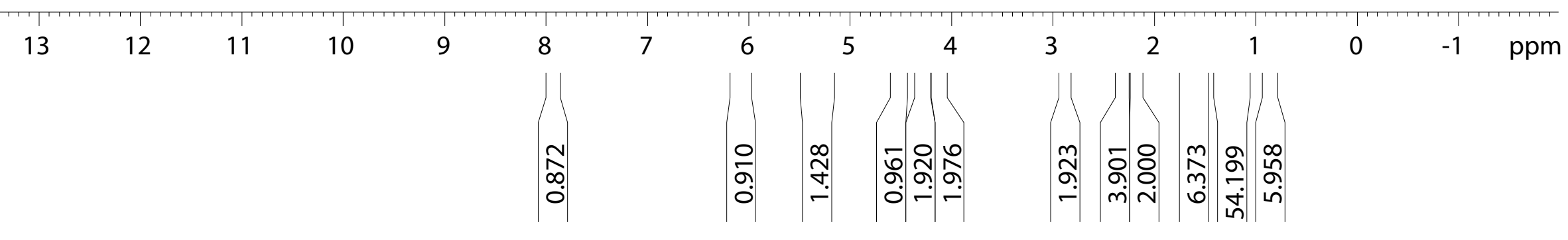




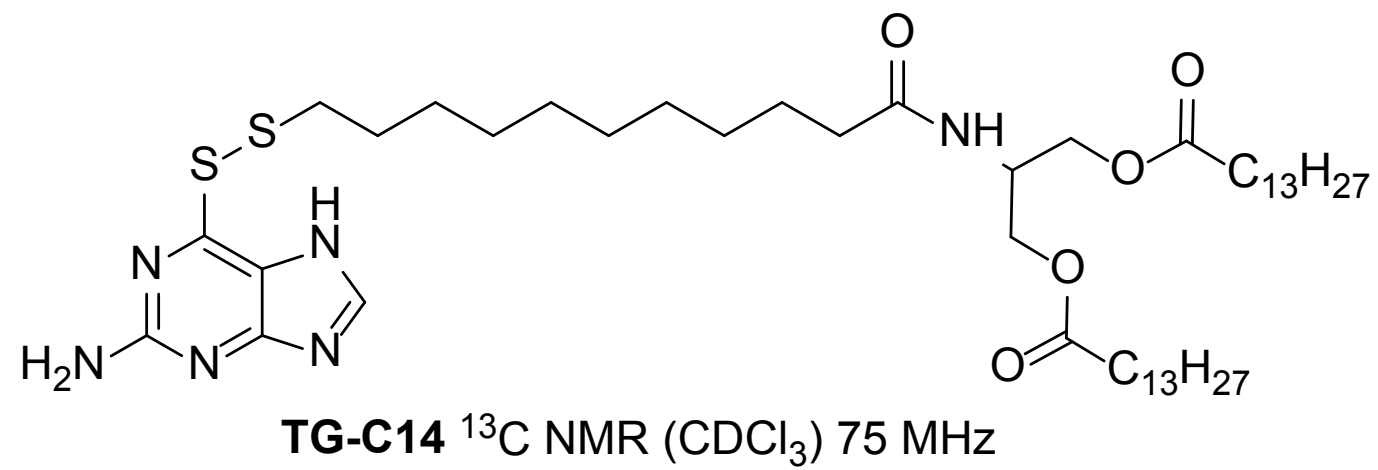




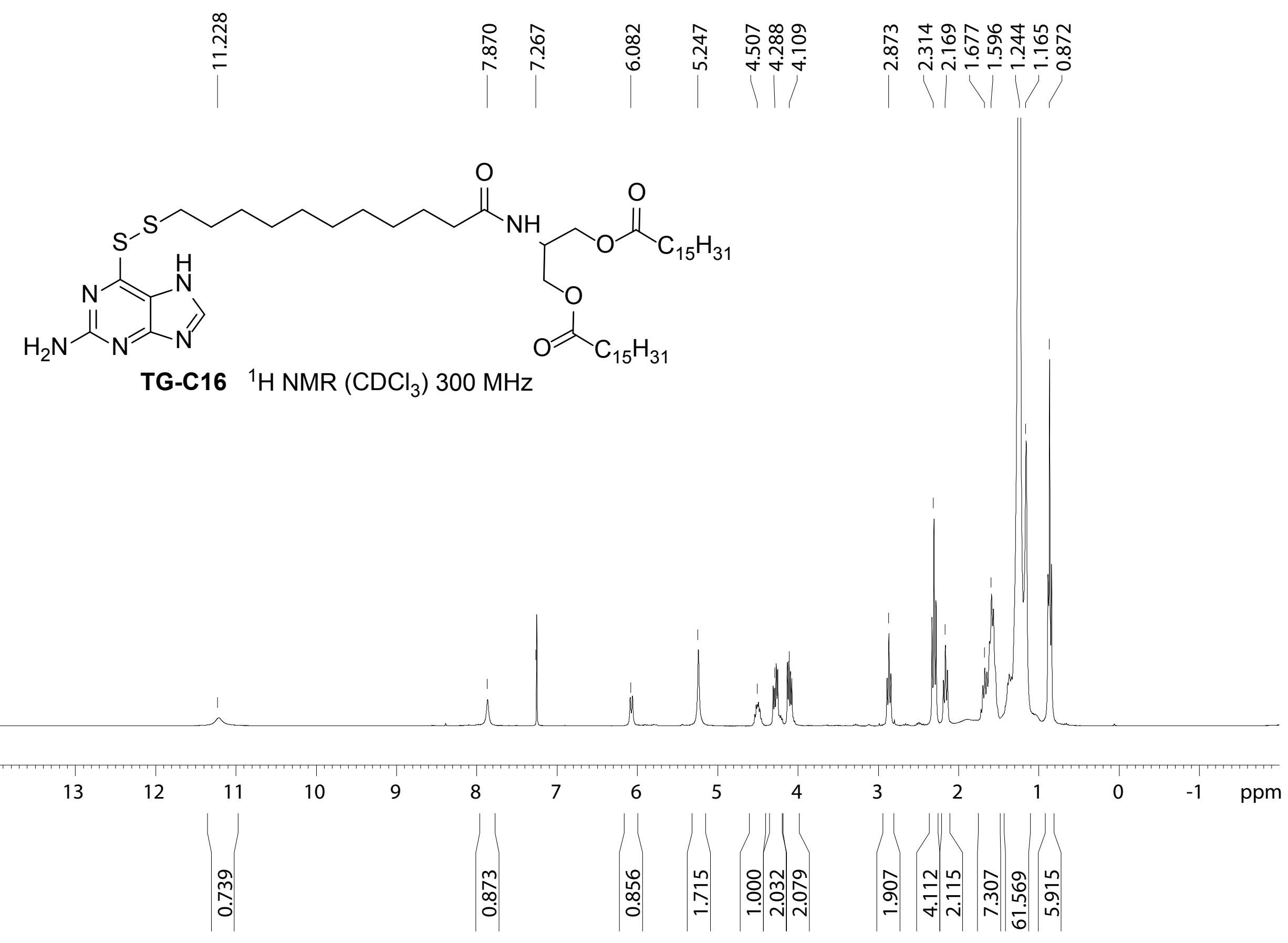




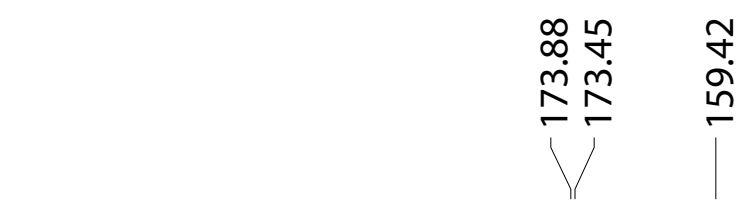

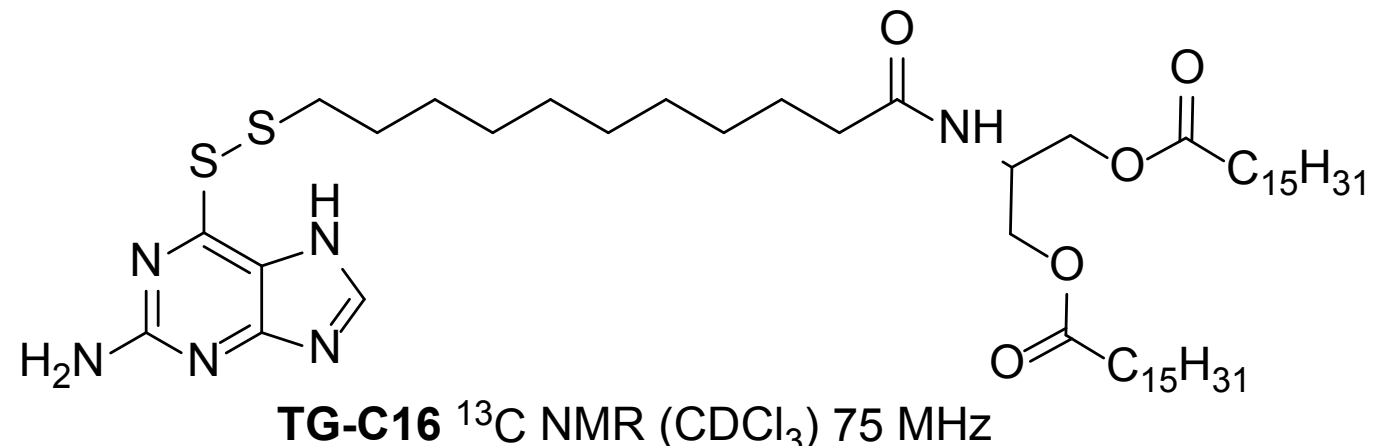

TG-C16 ${ }^{13} \mathrm{C} \mathrm{NMR}\left(\mathrm{CDCl}_{3}\right) 75 \mathrm{MHz}$

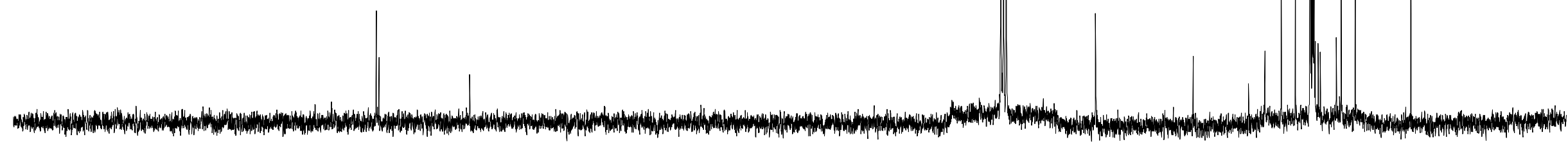




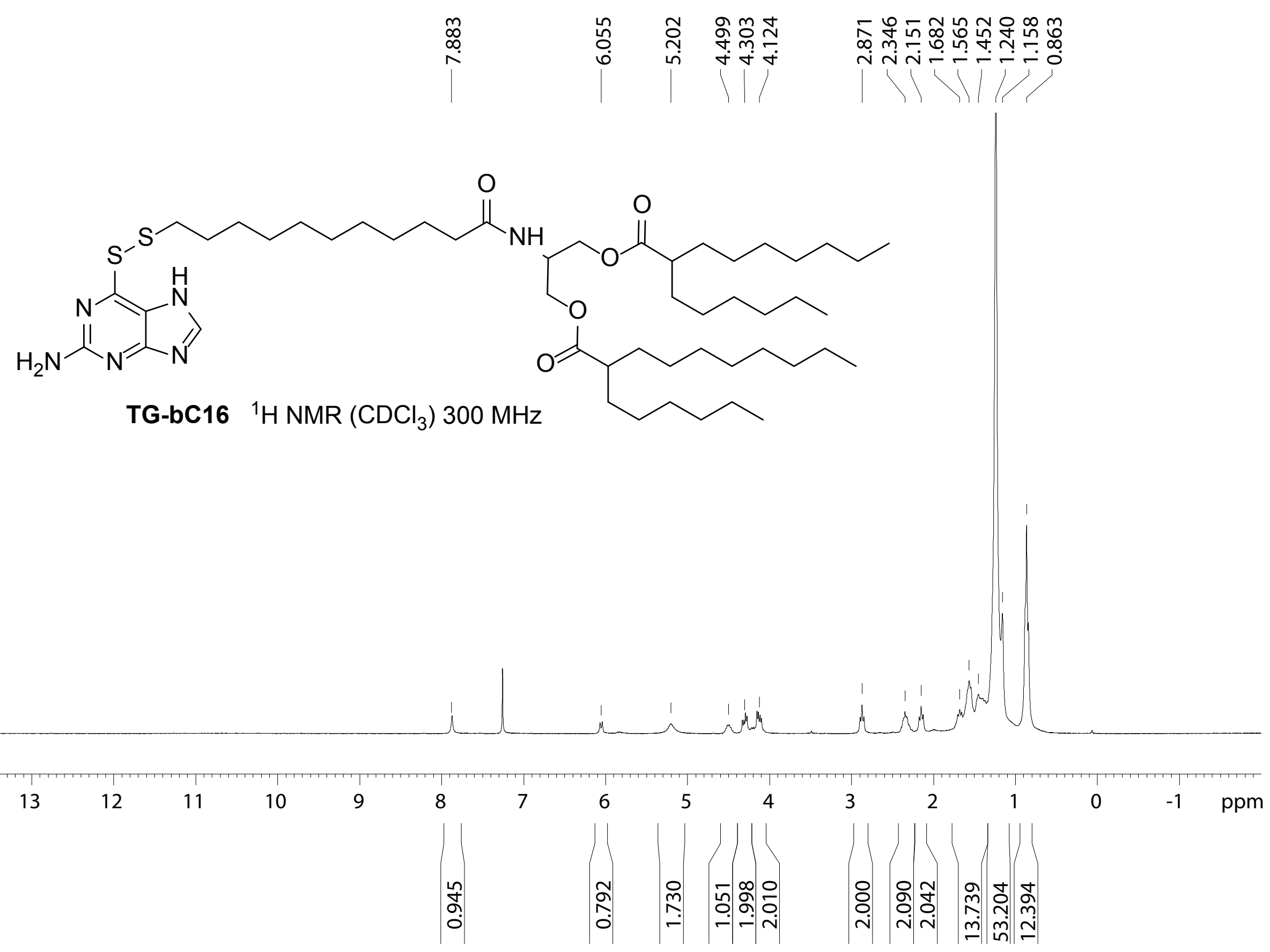




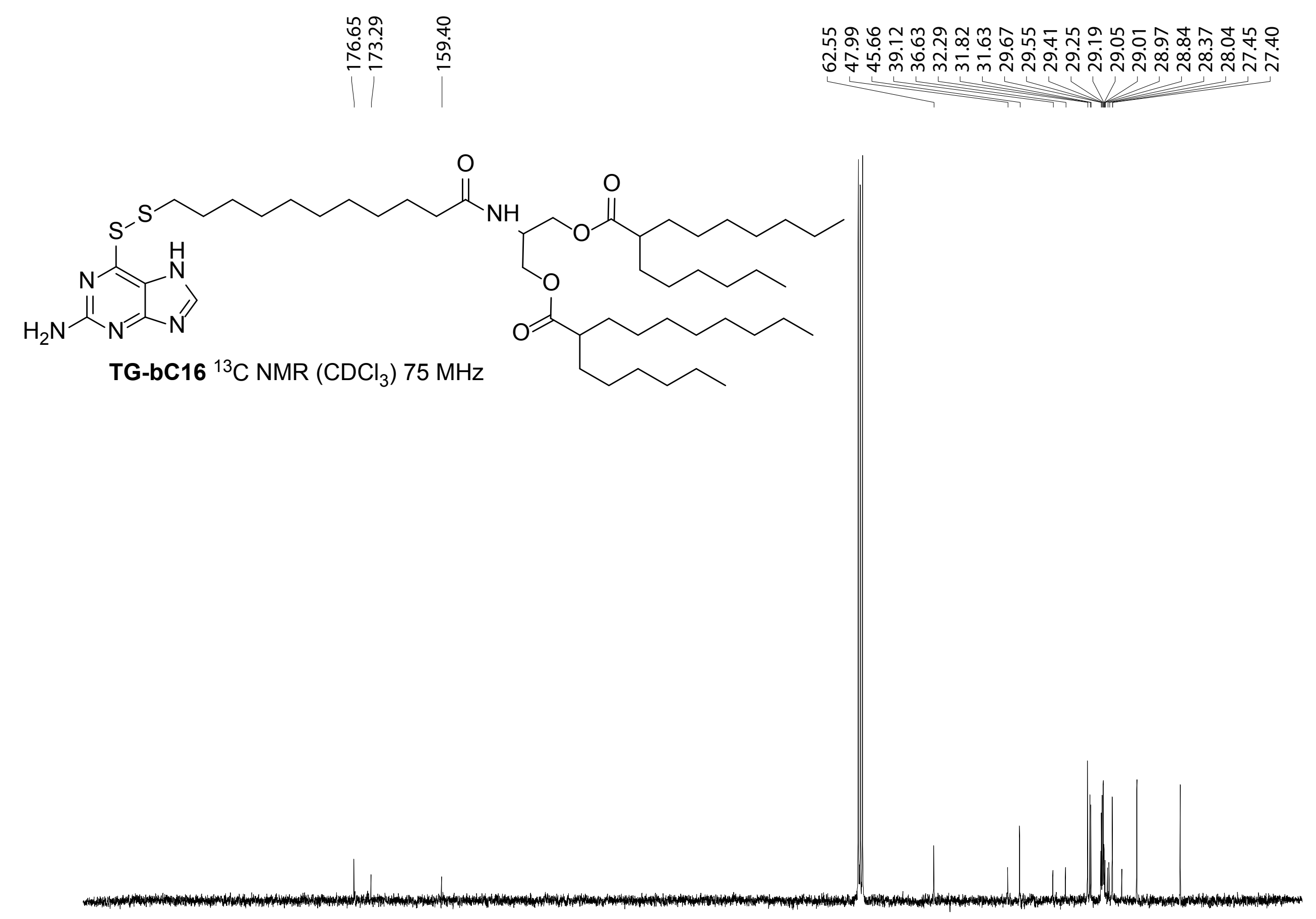




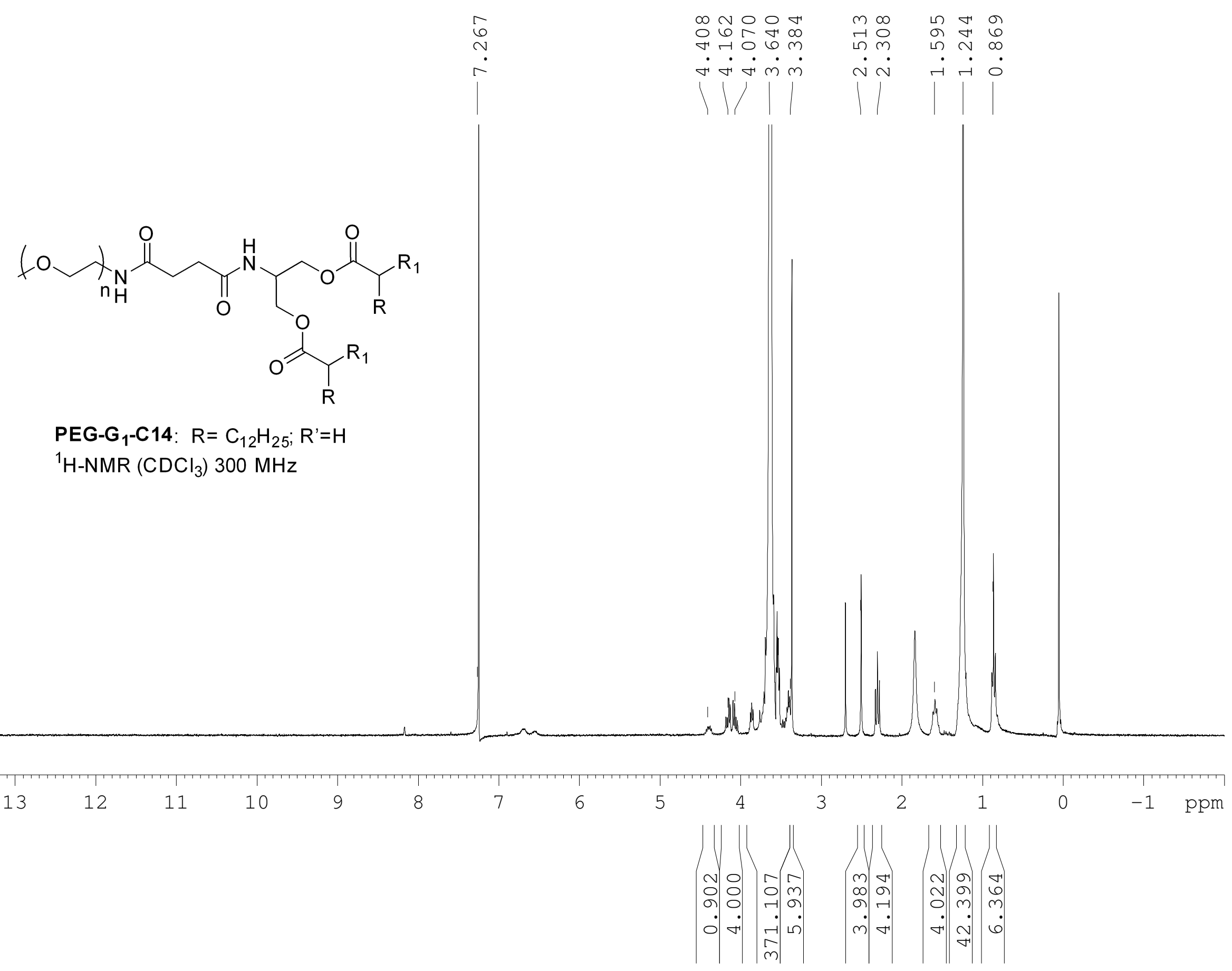




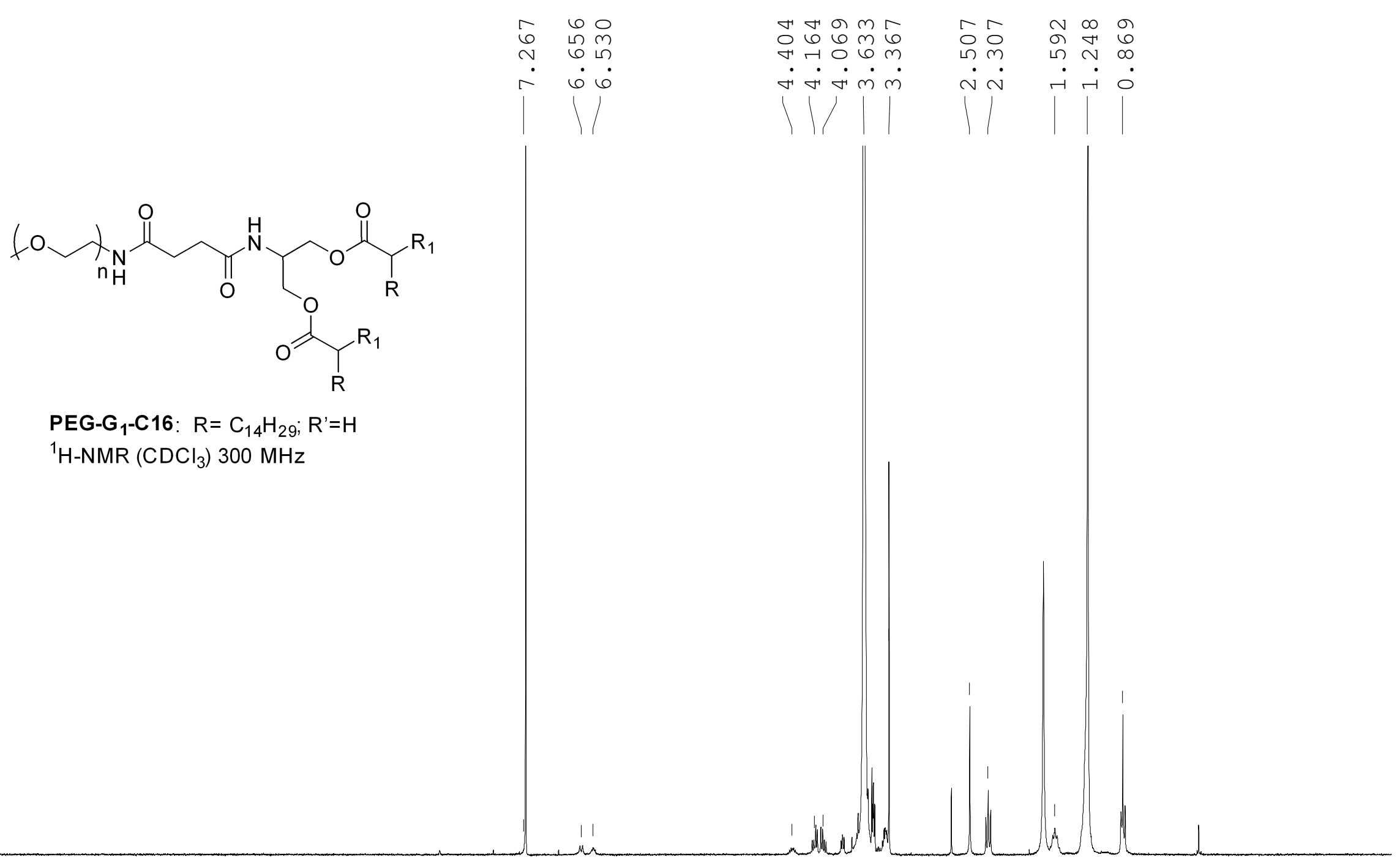

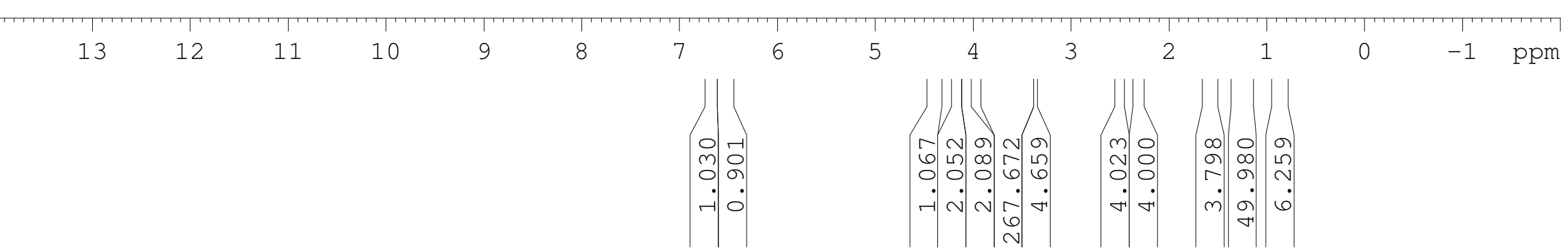




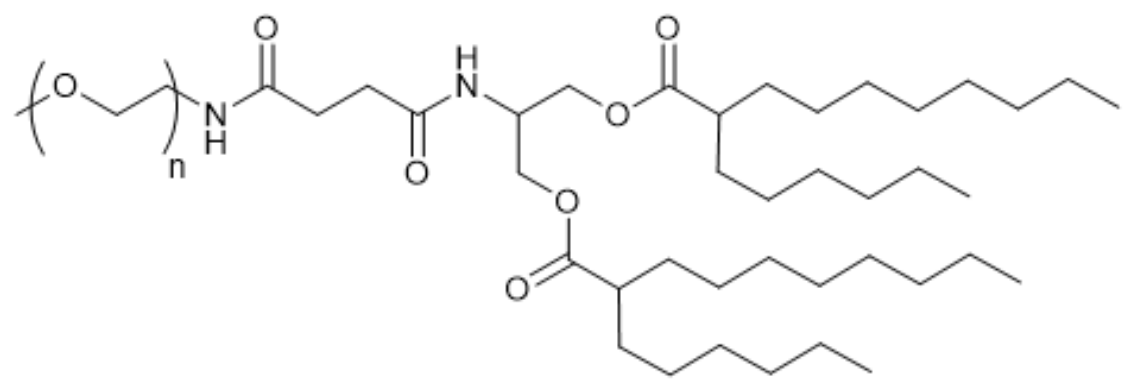

PEG-G1-bC16 ${ }^{1} \mathrm{H} \mathrm{NMR}\left(\mathrm{CDCl}_{3}\right) 300 \mathrm{MHz}$

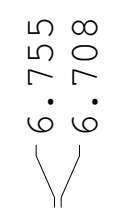

ஓ

म. 의

म

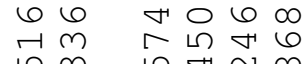

$\because$.

$\dot{i} \cdot \dot{i} \cdot$

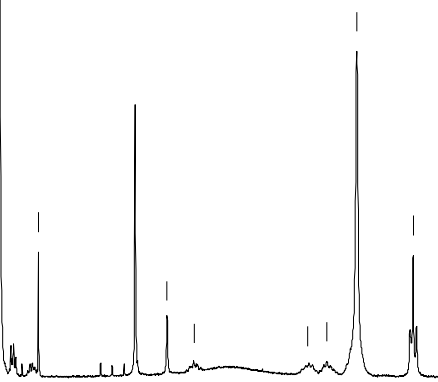

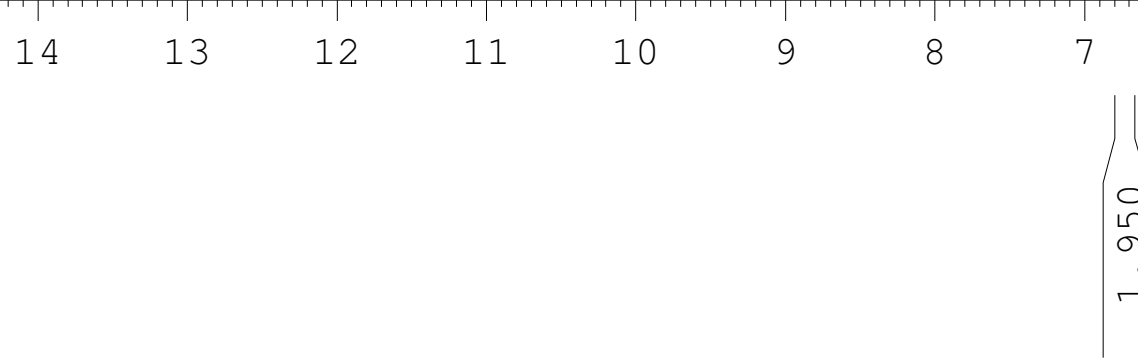

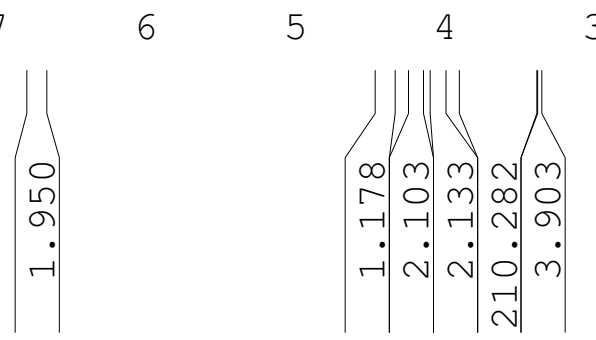

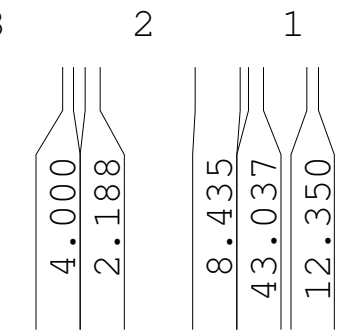




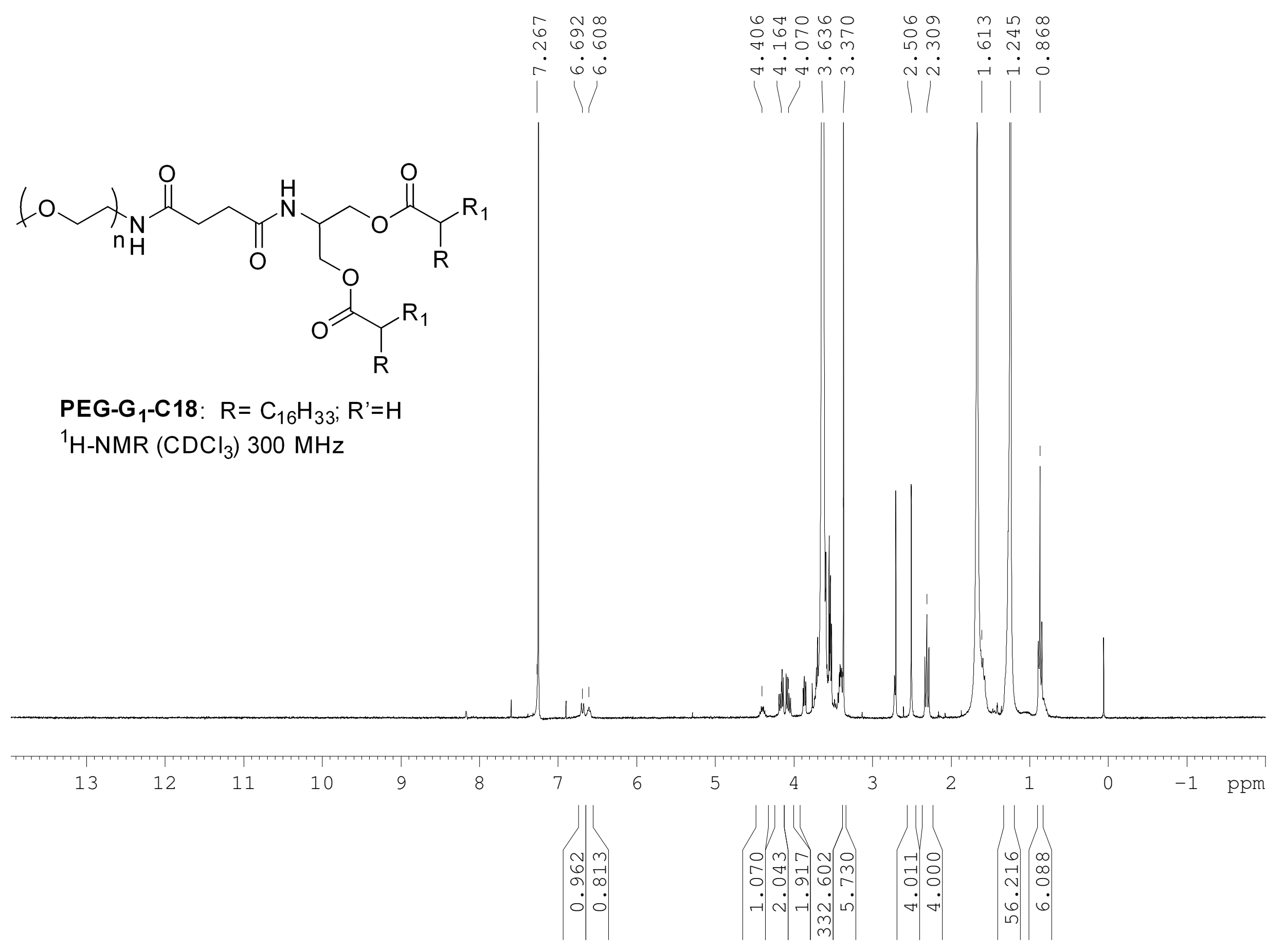




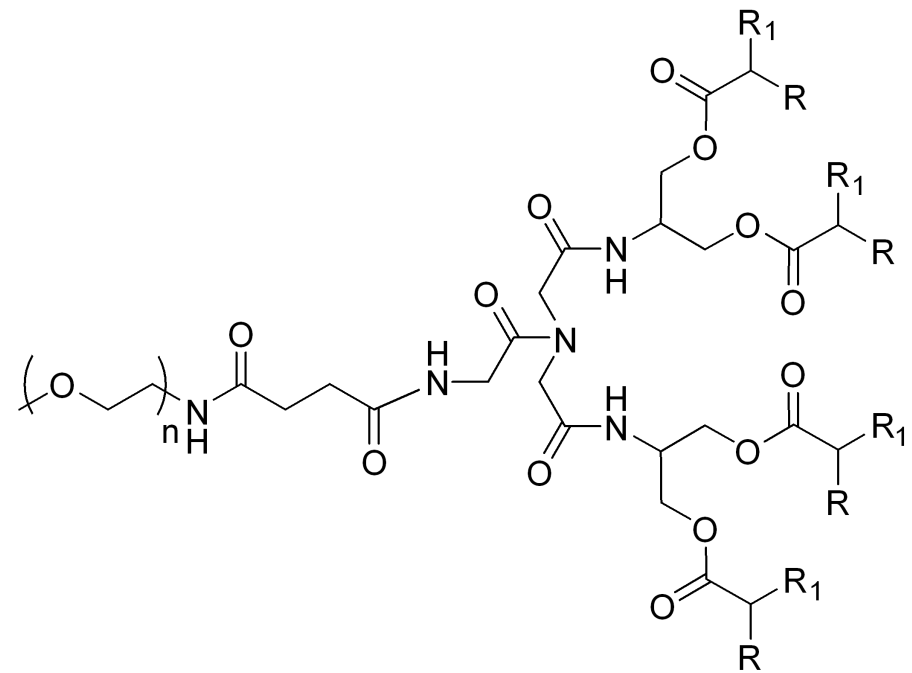

PEG-G ${ }_{2}-\mathrm{C} 14: \quad \mathrm{R}=\mathrm{C}_{12} \mathrm{H}_{25} ; \mathrm{R}^{\prime}=\mathrm{H}$

${ }^{1} \mathrm{H}-\mathrm{NMR}\left(\mathrm{CDCl}_{3}\right) 300 \mathrm{MHz}$

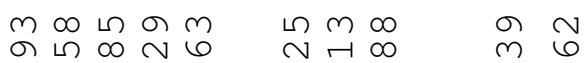

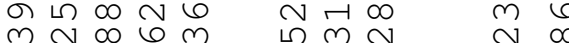

. . . . . . . $\infty \infty$

$\forall \forall m m \sim N \sim$

1111111 

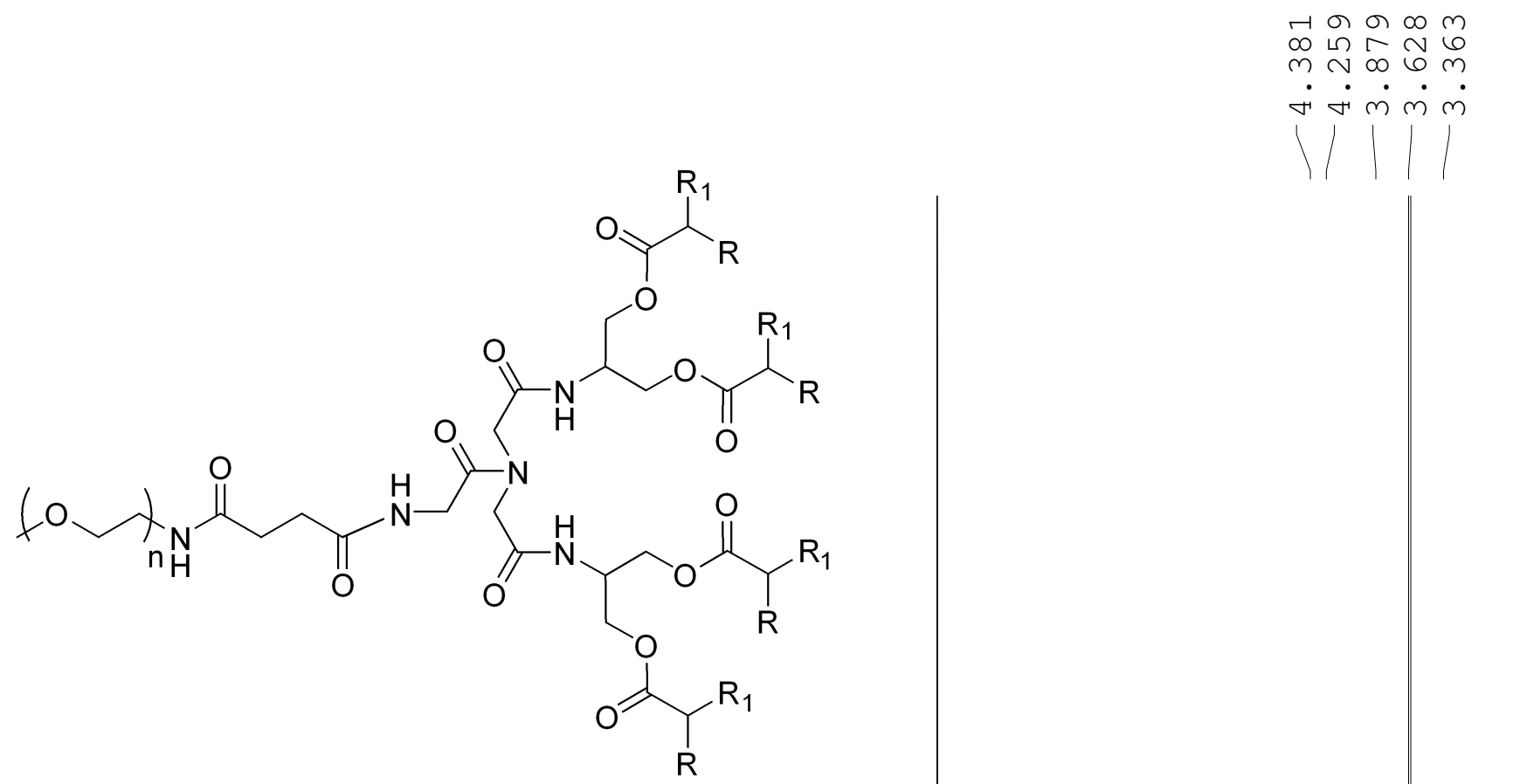

$\infty \sim$

ก

. $\dot{\sim}$

ก

$\forall \forall m m$

$\sim \infty$

$-10$

1111

PEG-G ${ }_{2}-\mathrm{C} 16: \quad \mathrm{R}=\mathrm{C}_{14} \mathrm{H}_{29} ; \mathrm{R}^{\prime}=\mathrm{H}$

${ }^{1} \mathrm{H}-\mathrm{NMR}\left(\mathrm{CDCl}_{3}\right) 300 \mathrm{MHz}$

11 1

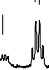

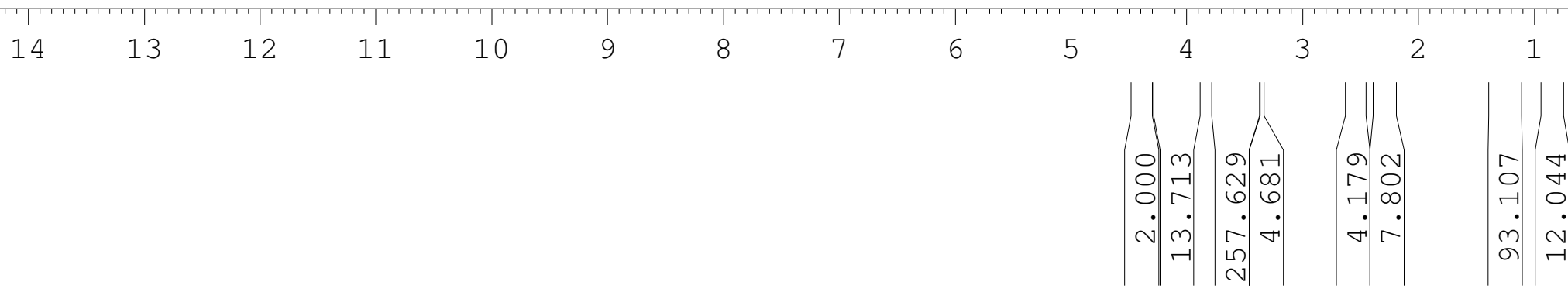




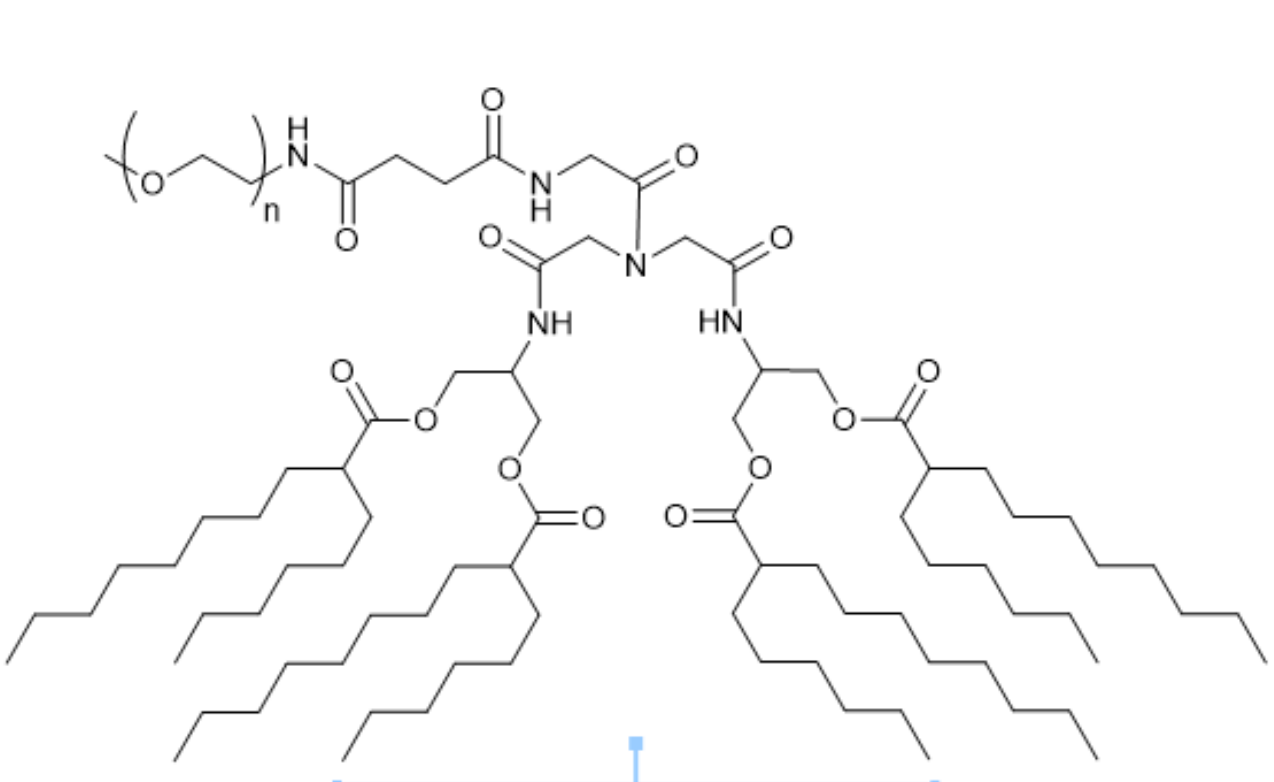

PEG-G2-bC16 ${ }^{1} \mathrm{H} \mathrm{NMR}\left(\mathrm{CDCl}_{3}\right) 300 \mathrm{MHz}$

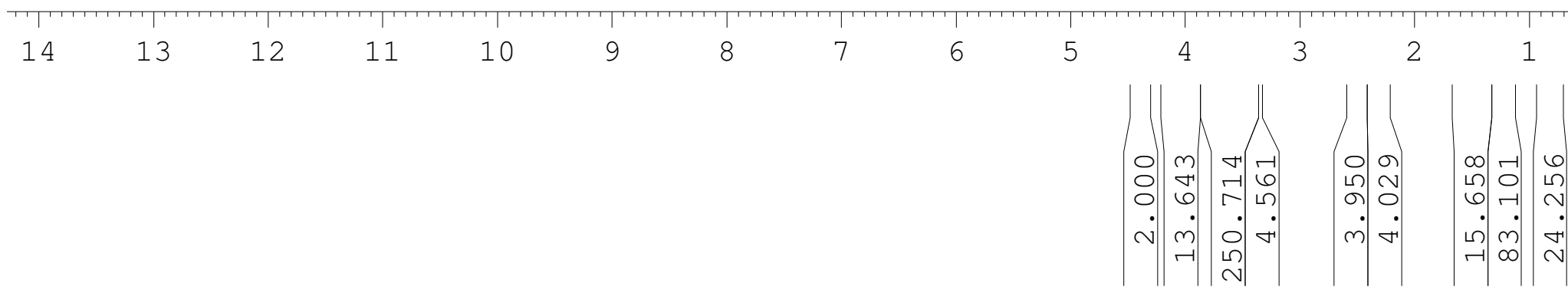



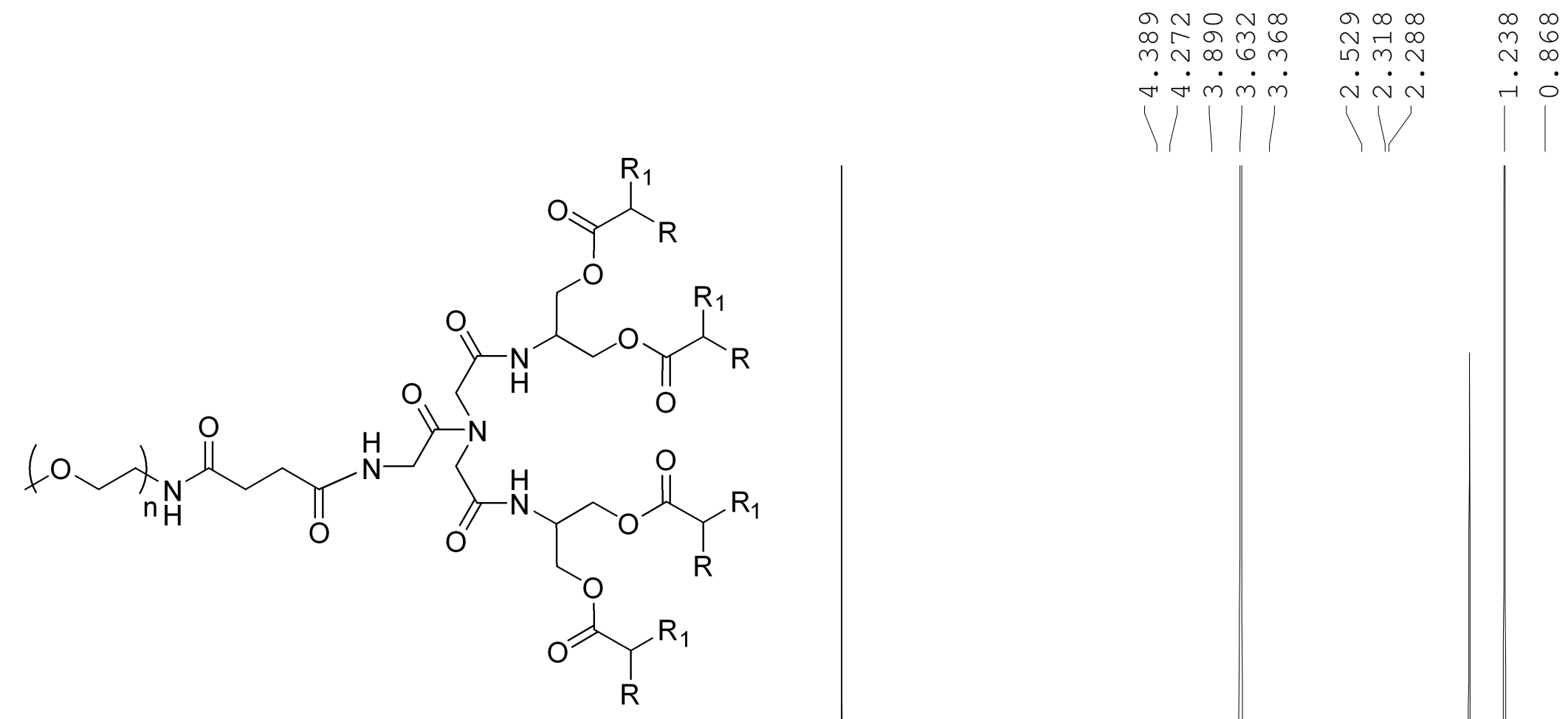

PEG-G ${ }_{2}-\mathrm{C} 18: \quad \mathrm{R}=\mathrm{C}_{16} \mathrm{H}_{33} ; \mathrm{R}^{\prime}=\mathrm{H}$

${ }^{1} \mathrm{H}-\mathrm{NMR}\left(\mathrm{CDCl}_{3}\right) 300 \mathrm{MHz}$

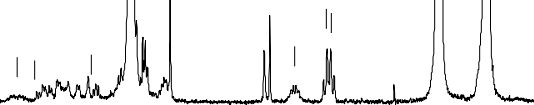

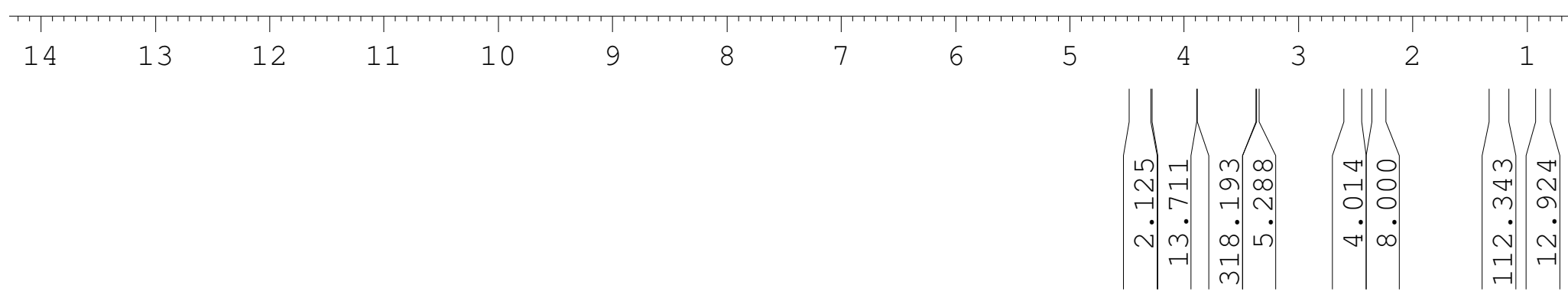

